$$
\ln \leqslant \sin
$$

\title{
Analysis of Abnormalities of Snubbers in Nuclear-Reactor Service
}

\section{(Report 1)}

J. H. Butler F. M. OHara. Jr.

Prepared for the

U.S. Nuclear Regulatory Commission

Office of Nuclear Reactor Regulation

Under interagency Agreement ERDA 40-495-7 Th 1 (I)

\section{OAK RIDGE NATIONAL LABORATORY}



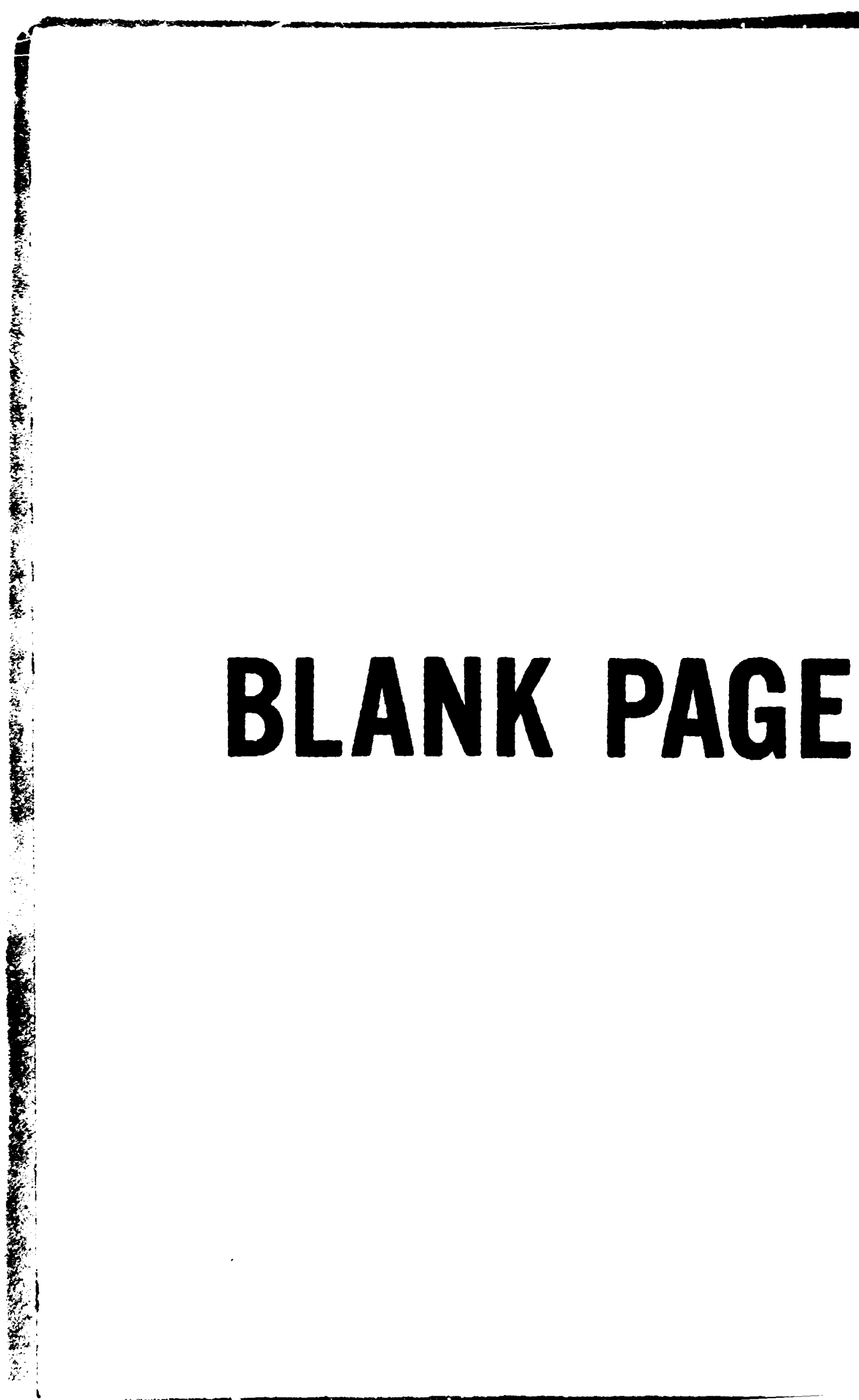
Printed in the United Sta:ts of America. Avialak's from National Technical Information Service

U.S. Department of Commerce

5285 Port Royal Road. Springtield. Virginia 22161

Price: Printed Copy \$5.00: Microfiche \$2.25

This report we prepered as an sccount of work sponsored ly the United seates Government. Nether the Unied States nor the Enerey Reserch and Developmem Administration/United States Nuctear Mequlatery Commussion, nor eny of their

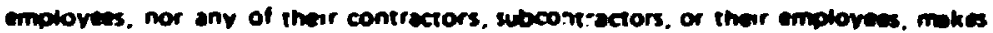
any werranty, express or implied, or axsumes any lepl inbility or responsibility for the eccurecy, completeness or usefulness of eny informetion. epporatus, product or process disclosed, or represents that its use mould not infrines porivetely ounded rents. 
OR.NL/.MTREG/TM-53

Dist. Categony SRC-4

Contract to. $\dddot{i n-7405-e a g-26}$

Eriguleering Technolog: Division

\section{ANALYSIS OF ABXCRMALITIES OF SNLBBERS}

IN NTCLEAR-:EACTOR SERVICE

\section{(Report 1)}

J. H. sutler

F. M. ' ' ara, ji.

Manuscript Completed: "Ictober 14, 1976 Date Published: :biemker 1976

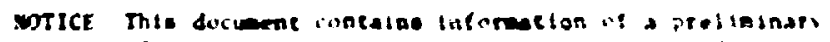
mature. It is sublect to revletun or cerretitin and therefore doese not represene tinel repure.

\footnotetext{
Prepared for ene

r.S. We lear Requlatory Camianion Mflise of Nuriear Reactor Rrzulation inder inceragency Arement man -0.-64; i-

Prepares by the nak Rioce suttoxul Laboutonr

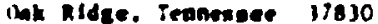
cpereted by

INIOW GMBIDE COAmation for the

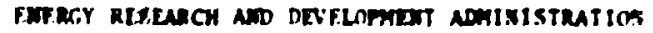

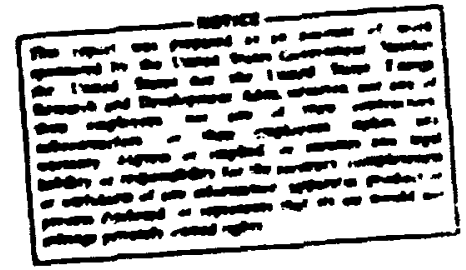




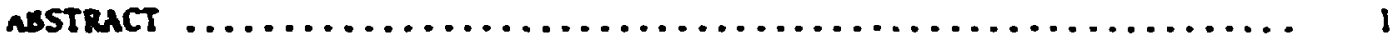

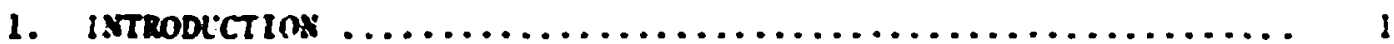

2. SNUEBERS IX GENERAL .............................

Functional Requirenente $\ldots \ldots \ldots \ldots \ldots \ldots \ldots \ldots \ldots \ldots \ldots$;

Hydraulic Snubbers ................................ s

Mechanical Saubbers ............................. 8

Typical Characteristics of Snubber. ................... II

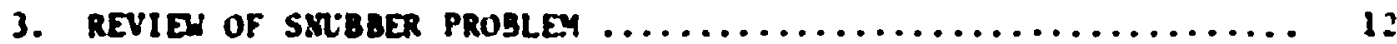

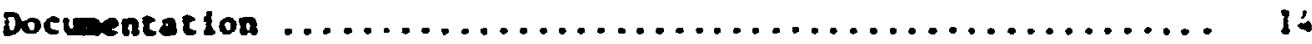

Abnomaliey Analysis ............................... is

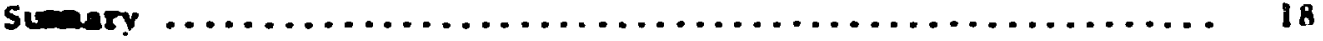

4. Coments and concLlsions $\ldots \ldots \ldots \ldots \ldots \ldots \ldots \ldots \ldots \ldots \ldots \ldots$ 28

5. meterduces $\ldots \ldots \ldots \ldots \ldots \ldots \ldots \ldots \ldots \ldots \ldots \ldots \ldots \ldots \ldots \ldots$. 29

APPENDIX A. MSIC BIBLIOGRAPHY OF FAILLTES OF SHOCK ABSORBERS

ALO OTHER SLPPORT STRUCTIRES (FAILL'RE ABSTRACTS) .... 3)

AP:ENDIX B. PQISTOLT OF RECON SEARCH OF ISA $\ldots \ldots \ldots \ldots \ldots \ldots \ldots$ 6 3

APPENDIX C. RO BLLLTIX \$. $73-3 \ldots \ldots \ldots \ldots \ldots \ldots \ldots \ldots \ldots$ 67

APPENDIX D. PO BLLETIN \$o. 73-4 ................... 69

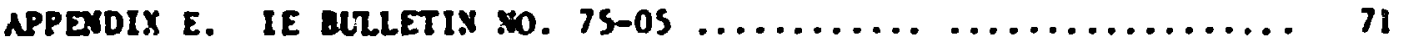

APPENDIX F. QUESTIOMRAIRE SERT TO REACTOR OPERATORS .......... 73 


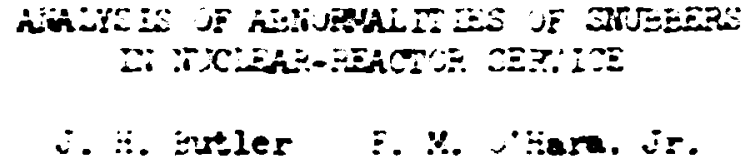

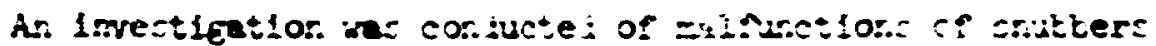
selsele-shoci arrestors 1. strice :t. :uclear poner flant:.

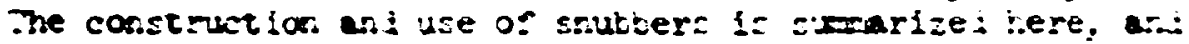
the t.1story of snubter srokler st. nuclear serfics is rejiewe:. seports of gang iundre is of snubcer salnusctions were found is.

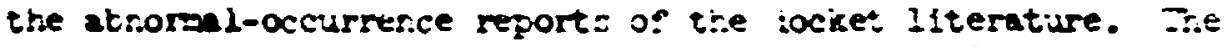

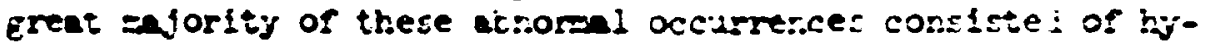
inulic snubber: whose hydreulic :lul i hai leaked ort tecause

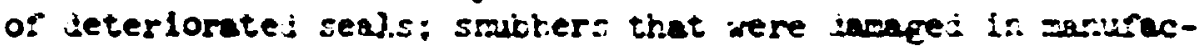

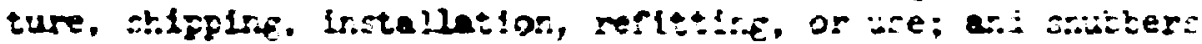
whose performance ild sot ontct: serice requirement. Adil-

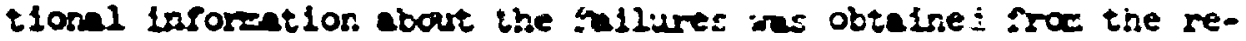
sctor operet lons, snubber sanufacturers. reator resjore, ani independent laboratories. The abnomal occiursances were clas-

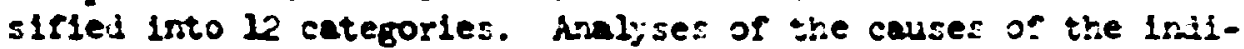

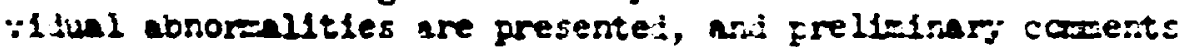

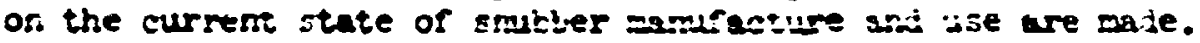

Lex rords: Smubers, shock aksorters, stock arrestors, selselc shock, plpe supports, restre ints, eartr.quakes, nuclear porer plants, impast shock, nuclear fiplne. Sellure, supjort stricture, blbliogrefty.

\section{DTRODETIO:}

The purpose of this report is to downent the results of ar. invest gation of nunctions of snubbers in service in suclear reactors.

A rash of wuch abnormel occurrences in 1973 caused the AEC $\mathrm{RRC}$ to 18sue a sertes of Regulatory Operations (RO) bulletins requiring reactor operators to inspect and rebulld certain hydraulic snubbers used in their piping systems. After this response to the :=eesiate conce.Tr, the Englneering Stas'ards Branch of the NTC decided to perforts a comprenenstve analvtical review of the performance of selsmic-shock arrestors ased in 
reactor facilitiles is the U.S. The Engineering Technology Division of ORIL has been zontracted to carry out this survey and evaluntion.

The scope of the orerall kRC program will be to review and evaluate smibbers and eventuaily to develop a regulatory guile for these devices. The ASEE, at the same time, is developing rules for the desion and use of smubers, which rules are to be incorporated into subsection IF of the ASIE Boiler ani Pressure Vessel Code, Sect. III.' The IRX progana will study present designs and alternatives, considering material somptibility with service enviroments and loading conditions, and the effectiveness of design criterie for smubber lock-up rates, free-floring rates, and bleed retes will be evaluated.

A meeting of OIrrL and IRC personnel was held on September 15, 1975, to discuss the smbber problem and to initiate a plan of investigation. It was decided that the infitial and primary consideration should be a thorough revitw of - allable information, as supplied by NRC, regarding snubter malfuncticn and design. The information provided by MRC has been revieved, and a flan of investigation has been formulated.

Activities in FY 1976 include the comprehensive review of serviceconditios abnormalities of both bydrulic and mechanical smubers with enpasis on failure-mode determination. Also, a review of the adequacy of methods used by architectural and engineering firms and reactor vendors to determine snubber specifica!lons will ve performed, and the design practices of smuber manufucturers will be investigated. The review and evaluation of smubber performance will be contimued into FY 1977.

completion of the above irvestigation should pinpoint design-practice deflaiencies ana inadequacies, $2 s$ related to snubber fallures. This information will set the focus for a thorough evaluation of analytical methods and their correlation with experimental data in order to determine the role of the ruactional variables in smuber design. The feasibility of data extrapolation fram small- to large-capacity snubbers w1ll also be investigated. It should be noted that, as it stands, this phase of the investigntion relies solely on avallable test data but that successful completion of this work my require rather extensive experimentation by independent Investigaturs. 
The cumulated results of the preceding research should provide a reliable means of developing recomended test procedures far smubers to be performed by the manufacturer, user, or independent sources. The ultimate objective for this frogram is to develop information to contribute to the development of regulatory gidies, codified standards, qualityassurance practices, and in-service inspection procedures regarding smubbers.

The plans for scheduling, conducting, and reporting these activities are outlined in Table 1.

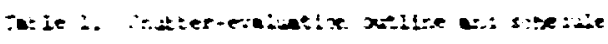

$\div:$

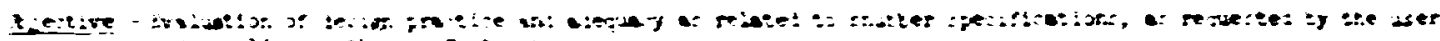

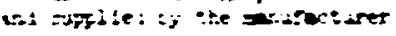

is:

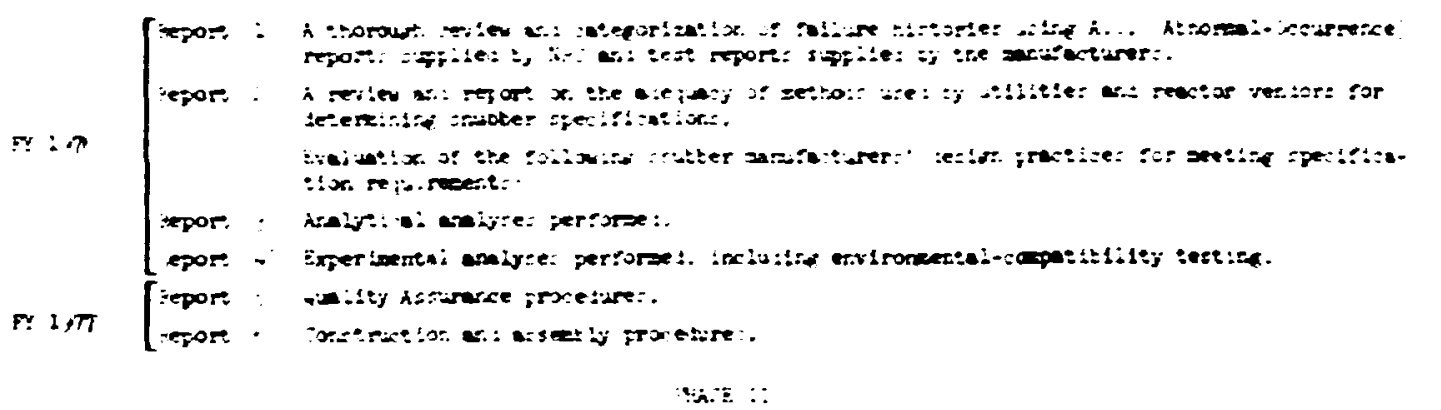

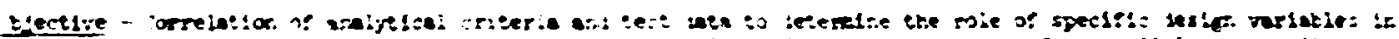

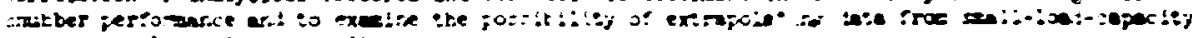

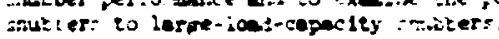

iles.

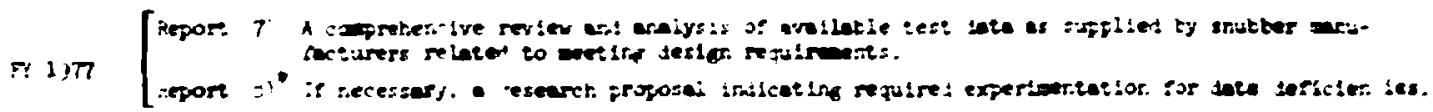
was :::

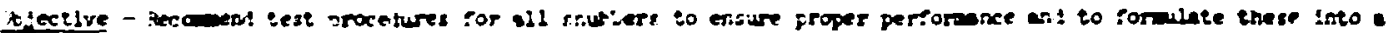
regulatory oul te

Inen

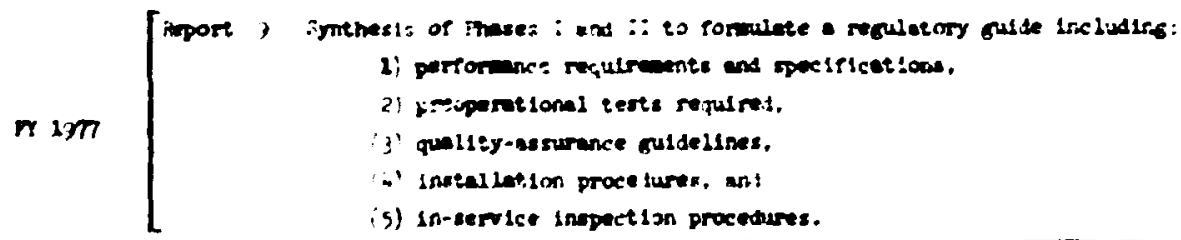

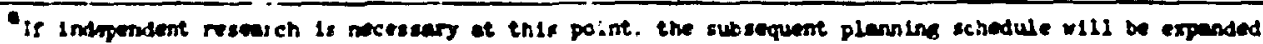
eccordingly. 


\section{SNUBBERS IN GENERAI}

\section{Functional Requirements}

Smubbers are devices that would absorb the shock of an earthquake for pipes or for piping components in a melear-reactor systen yet still freely allow the smaller and slower morements caused by heating and cooling of such pipes or components. Smubbers are jesigned to be attached to a fiping component that is considered vulnerable to earthquake damage and to allow that component to move freely within certain limits of rate and extent of movement. If these linits are exceeded, the smubber locks up and temporarily provides rifid support for the component until the force acting on it ceases.

Ficture a length of straight pipe rigidly attached at both ends. If the pipe and its supports were rapility and cyclicslly accelerated by, say, the grourd aotion of an earthquake, the pipe would bend under the dyramic lond, and the stresses in the pipe might exceed the yield strength of the material, cassing gross deformation or rupture. A srubber installed at the midpolnt of the pipe would, upor locking up, halve the harmonic-response lenth of the pipe and essentially double the amount of energy needed to prociuce gross deformation or rupture of the pipe.

If seismic restraint for such a pipe were provided by rigld supports, the thermal behavior of the pipe and the frequency-response level of the piping system would be altered. This would also generate st.-sses that could damage the plping system.

Smbbers, then, must accamodate movements of a component resulting fram thermal expansion or system vebration but at the same time ve capable of rigidly restraining that cumponent in case of selsmic shocks. Moreover, they must allow for superposition of these two modes of motion, as, for example, in the case of a dynamic displacement force followed by a rapid thermal transient in the same direction. This necessity requires the smubber to be designed to release rapidly from the locked-up mode or at least to orerride the lock-up rigidity to accomodate subsequent thermally induced motion. 
There are $t w$ basic types of smubbers that fulfill these requirements, hydraulic and mechanical smubbers. The reminder of this chapter will discuss the various designs of each of these types of smubbers.

\section{Strawlic Smubbers}

Hydraulic smubbers absorb shocks by compressing a nuid and forcing It through a flow-restreining device. A prototypic hydraulic smabber consists of a rod attached to the piping component to be controlled with the other end of the rod attached to a piston in a piston chamber fuil of fluid. Under campression, the fluid exits fron the chamber through a flowrestraining valve and goes to an accualntor reservolr or beck to the piston cheaber on the back side of the piston. The body of the smubber is securely anchored. The basic desion of a hydrulic smuber can be seen in Fig. 1. Snubbers can have single- ar double-ended plston rods, depending an the application see Fig. 2). The advantage of the double-ended piston is that the volume evacuated on ase side of the piston is the same as the volume displaced on the othar as the piston travels through the chamber. This lessens the role of a reservolr or an accunulntor to singly accomodating the thermal expansion of the hodreulic Fuid and making up flutd lost through leaks. A photograph of an actunl scubber is shown in Fib. 3 .

The flow-restraining valve 18 designed to close the fluld passages wen the flow rate exceeds a given limit. In the design of F1g. 1, this closure is accoulished with a poppet valve. Lock-up occurs when the pressure caused by the nuld now exceeds the opring force and the valve seats. With the valve closed, travel of the piston 18 linited by the campressibilIty of the slusd and the magnitude of the dymic-displacement force. When the force on the piston rod is reacod, the pressure on the ralve is relieved, and the spring-20aded poppet valve opens again, returning the unit to its normal condition. Another poppet valve prevents rapid flor of flutd in the opposite direction. Such smubers handle cyclic loads of up to about 30-Hz erequency.

In case thare is an extended loading on the platon rod, alow presoure-reilef cepability is buslt into the smbbers. This nifit consist of grooves in the seating surfuce of the poppet valve or an auxiliary orifice. 
ORNL-DWG 7b-16880

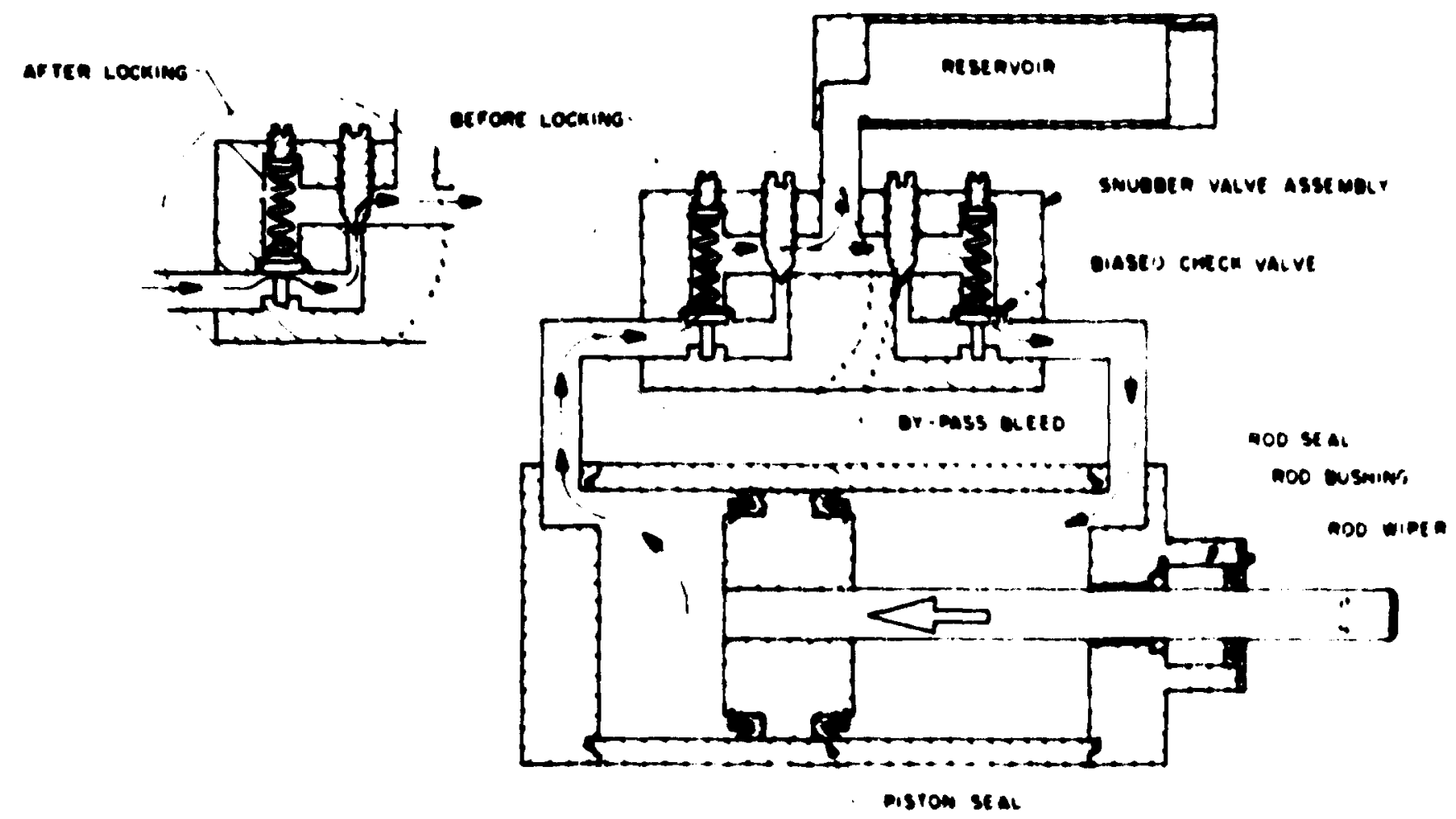

F1g. 1. Schematic 1.ulustration of a hydraulic shock errestor in operation. Drawling courtesy of IIT-Grinnell. 

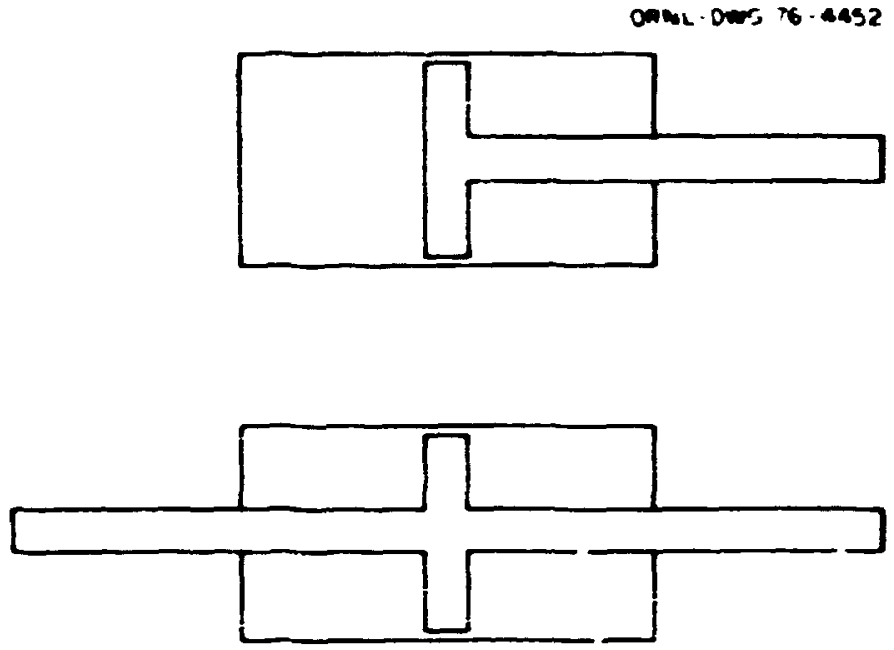

F16. 2. Dlustration of (a) single- and (b) dcuble-ended pistons.

PETTO $5775-76$

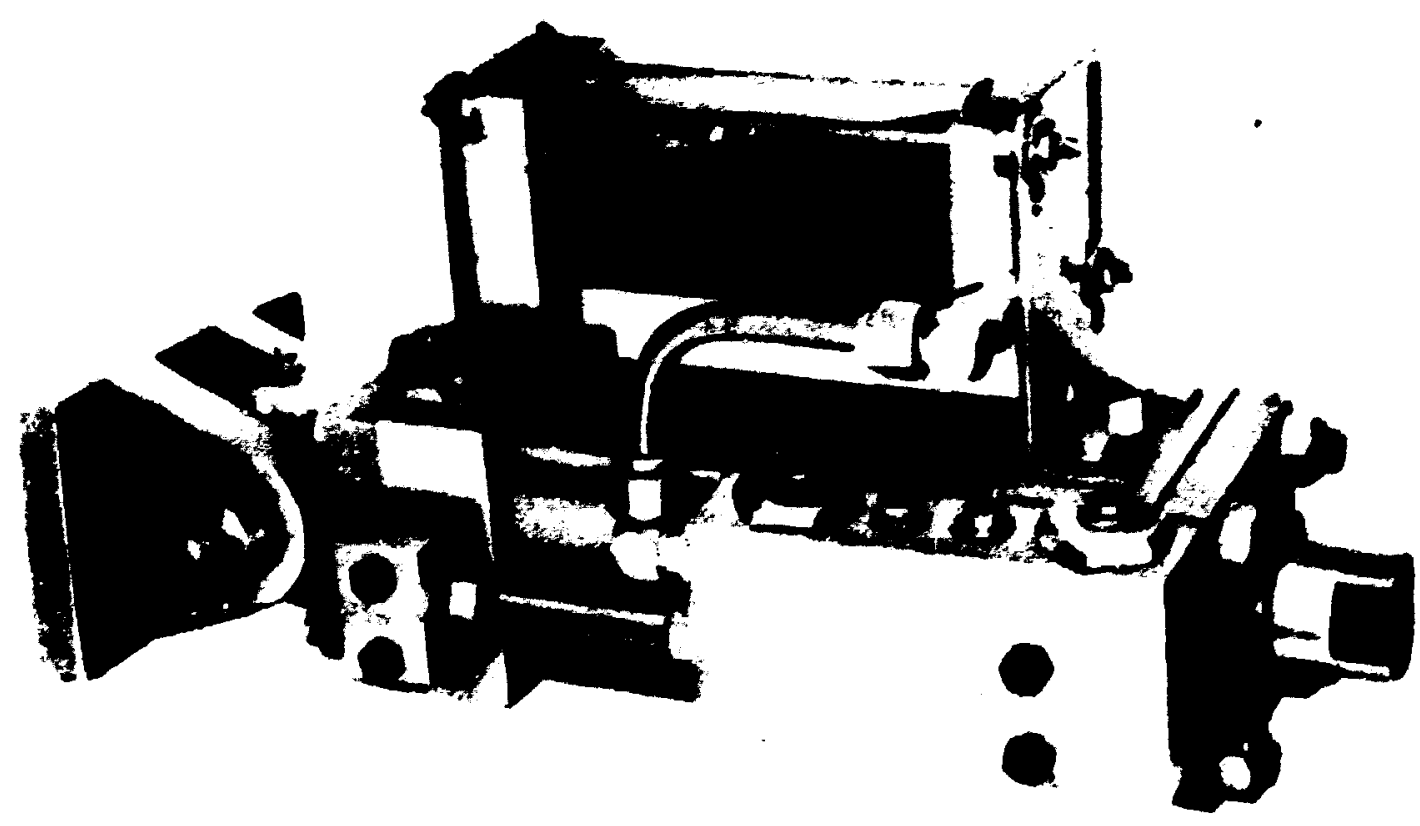

Fig. 3. A wodralic subber with waraulic-fluid reservoir on top, mounting assembly to the left, pieton rod extending on the right, and hydraulic cylinder in the center. The valve assembly is seen on the rifint front of the device. Photo courteny of IIT-Grinnell. 
Very slowily, tinis allows the smibber to bleed off the pressure vithin the hydraulic cylinder and to absorb the sorce on the supported piping component. The bleed : ate is variable, ant the maximum trevel of the piston is linited, of course.

The majority of a hydraulic smubier is of steel construction, seals are generally of polymeric material, and the hydraulic fiuid is usually a silicone oil that has been selected for its resistance to temperature and radiation.

\section{Nechanicel Srubbers}

Mechenical smubbers adjust to thermal motions by telescoping, and they contain devices that losk up when the telescoping actirn occurs too q.2icicly. A variety of mechanical devices ie.g., springs, ball srrews, rotating masses) are used to translate the axial telescoping irto angular acceleration and to control (that is, to limit) that axial velocity. Four of these design concepts will be discussed here.

The simplest device is just an inertia mass (flywheel) attached to a screw. The freely rotating screw is attached at one exid of the smubber, while a ball unit is rigidly attached at the other (see Fig. 4). Slow telescoping of the smuber accelerates the wass, whereas the aevice would not have time to react to a transitory and rapid axial compressive force, acting instead as a rigid structure.

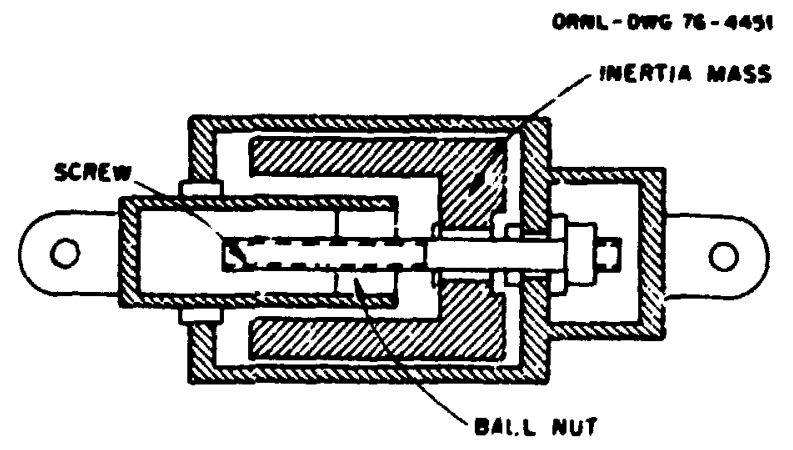

Fig. 1. Simple inertia-mass-smubber desion. 
A second device that uses a ball mut and screw to accelerate a rotating component employs an element with a low coefricient of friction between the rotating component and the (stationary) snubber housing. At low rates of axial conpression, this friction element will allow the rotating member to slide over it; at high retes, it will not, and the snubber will lock up.

A more sophisticated version of the latter device employs a sprirgloaded friction plate attached to the central. rotating member (the ball mut;. Upon rotation, this rriction plate expends outward by means of an adapter (see FIg. 5). When the centrifugal force of the rotating friction plate exceeds the force of the centering springs, the friction plate contacts the smubber housing, and the unit locks up. If the axial load is unidirectional, the ect of locking up eliminates the centrifugal force, allowing the smubber to release and re-engage cyclically until the longterm force is accomodated.

The final design concept to be discussed here is illustrated in Fig. 6. In this design, the telescoping action of the init accelerates a drum assembly that is coupled to an inertia mss by a resilient capstan spring. When the drum assembly mores, it turns the inertia mass by twisting the spring. If the inertia mass's resistance is too great, the spring tightens around a mandrel, stopping the rotation of the drim and locking up the

ORNL-DWG $76-4453$

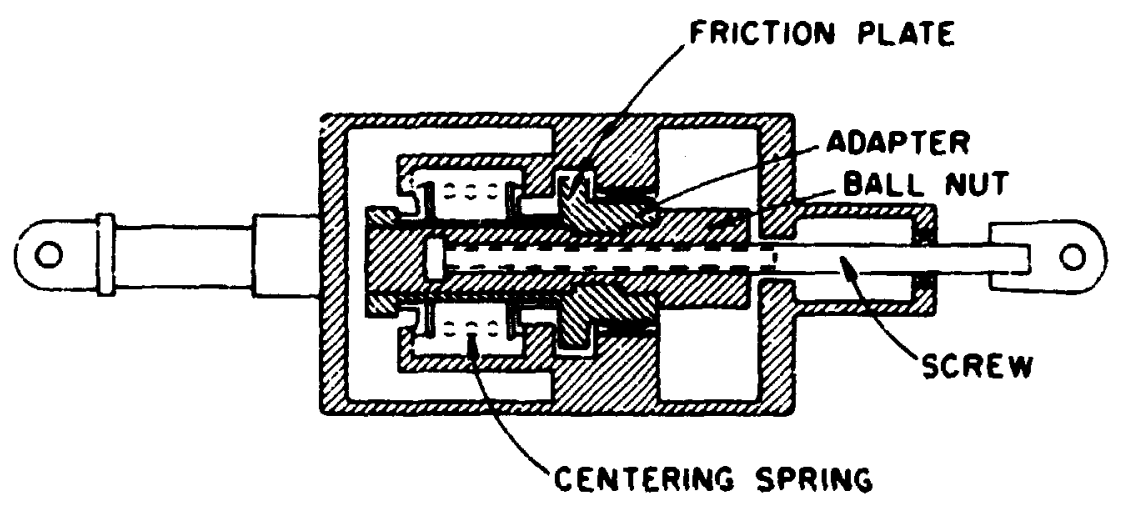

F1g. 5. Snubber design utilizing spring-loeded friction clutch. 


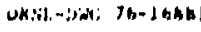

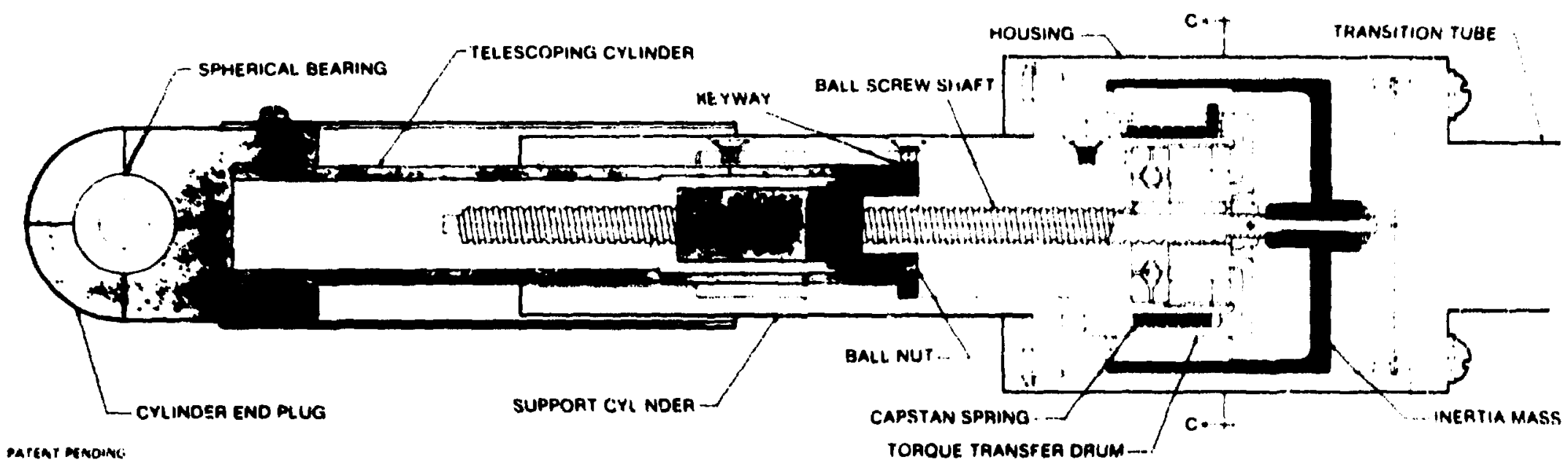

Fig. 6. Schematic illustration of a mechanicel shock arrestor. Drawing courtesy of Pacific Scientific. 
snubber. Due to sy-netry, the sane capstan spring will brake the drual screw under both tensile and conpressive loads. Once the device bas locked up, the freely turning inertia mass will slowly accelerate and relieve the torque on the spring, releasing the mechanism and alloring it to recycle if need be. Again, the net effect is an iterative throttling and braksing that limits and controls the axial acceleration. The limiting factor is, of course, the stifmess of the capstun spring, which is chosen so that the unit car provide normal themal courensation without tightening the capstan brake.

The muterials used in the construction of all mechanical (as well as hydraulic) smubbers must confors to the ASrE Code, I Sect. III, Appenaix I, and Code 3ase 1644 (Rer. 2). The Code does not cover seals, springs, wear shoes, lubricants, retaining rings, and bearings there. Such items must be designed to withstand the effects of the enviranmertal temperetures, muidity, and rediation doses encountered in reactor service.

\section{Typical Charecteristics of Smubbers}

Off-the-shelf smubbers are arailable with load ratings from $2.2 \times$ $10^{2}$ to $8.9 \times 10^{6}$ newions (50 to 2,000,000 1b). These are the maximm forces that the smubbers themselves will withstand. If a strut assembly is used, column strength must also be considered along with the properties of attachment devices.

The stroke or travel length of a smubber can be of any reasonable magnitude, and the length will vary from mamufacturer to manufacturer. A proposed rule $e^{3}$ is that the minimum stroke length shouli be the maximum calculated thermal movement plus $20 \%$ or 2.51 m ( 1 in.), whichever is greater.

Smubbers typically lock up when the axial acceleration exceeds a limit set between 2.54 and $25.4 \mathrm{~cm}$ ( 1 and $10 \mathrm{in.}$ ) per min ${ }^{2}$. Furthermore, iterative travel of the piston rod upon cyclic loading ( 3 to $30 \mathrm{~Hz}$ ) is generally limited to less than $1.5 \mathrm{~mm}(0.06 \mathrm{in}$.). This acceleration limit corresponds to an imposed force of less than $2 \%$ of the unit's laad rating. Relief of extended-term loads typlcally proceeds at the rate of $12.7 \mathrm{~cm}$ (5 in.) per min ${ }^{2}$ depending on the epplied lond and operating temperature, 
although high-capacity units may here rates as $10 \mathrm{w}$ as $0.1270 \mathrm{~cm}(0.05 \mathrm{C}$ in.) per min m. $^{2}$.

Load ratings of smuber components (relief valves, screws, etc.) typically exceed their smuber's load rating by 120 to $230 \%$.

\section{REVIBN OF SIUBBER PROBLB:}

Cancern about the safety and dependability of smibbers in ger.eral can be traced back to the malunction of a muber of hydraulic smubbers in the Millstone-1 unit. The finding that 51 of 112 hydraulic snubbers were defective because of a loss of hodraulic fluid was reported in an abnormal occurrence (AO) report in July 1973. This 10 report is abstracted in citation 10. 118 in Appendix A. " The Millstone-1 incident resulted in the issuance of Regulatory Operations ( $\mathrm{RO}$ ) Bulletin 73-j, which is included here as Appendix C. This RO bulletin asked reactor operators to survey the status of any and all Bergen-Patterson hydraulic shock absorbers at their installations. The response to RO 73-3 indicated thet similar problens were occurring in many reactors, and RO Bulletin 73-4 was issued requesting operators to make desalled inspecticns and analyses to determine and rectify the causes of the problems. Appenitix D shours $10073-4$, and the replies to RO 73-3 and 73-4 are included in the sumary table preserted later in this report.

The reactor-operators' investigation showed a high rate of seal deterioration and failure in the hydraulic smubers. This prompted BergenPatterson to perform an investigation of the performance and desion of the seals in their snubbers. They issued a report of their findings in actober of 1974, including the recounendation that the millable-gum seals, wich were the original equipment in the Bergen-Patterson smbbers, be replaced with seals made of ethylene propylene.

An 10 report (printout No. 15) 1ssued by Metropolitan Edison Co. for its Three Mile Island 1 reactor indicated that eight smubbers were found th.at would not lock up at the specified rut.e. In response to the Three Mile Island incident, If Julletin 75-05 was issued, and it is included

*Hereafter, cications listed in Appendix A will be referred to by the mubers associated wth them in that printout. 
here as Appendix E. In response to RO 75-05, Grimall evaluated the 1xckup problea and issued a report in Aprll 1975 (Ref. 5). Their report inilated thet the problens were caused by the wrong hydralic flutd being installed in the smubbers and oy the presence of couteninants in the Nuid. Grinnell recomended that a specific fluid be used in the smubbers.

The American Society of bechnical Bngineers oreanized a Task Force on Snubbers. This Task Porce issued a prelfiningy report in yay 1975 (Ref. 3) that provides inftial-draft material designed to forn the basis for specific recomendations for charges to the ASrz code, Sect. III, 1 subsect. IF. This draft covers definitions and descriptions of surbbers, loads to be sustained by swubbers, application and installation of smbbers, startup and testing procedures, specifications, and in-service inspecticn of smibbers. Other sections of the draft are still in preparation. It is expected that the codification of smibber desion, specification, andysis, use, and inspection will oblate aw probleas with smubbers.

The Huclear Regulatory Conission also felt sane concern with the manfucture and use of saubbers and contrected with Oful to investigate smubber performace criteris. The rest o: this report deals with the initial results of that evaluation of subber perforsance.

It should be equhasized that the purpose here is oot to ma speciflc recomendations about preventing or correctiog av fullure of subbers in maclear service. Rather, this repout is coscerned primeliy with the determination of the srequency of fallure and nodes of fellures of ambibers In muclear service and with the provision of a coppobensive evaluntica of those ratlures. The word "fullure" here does not necesenrlily mean thet the smubber was found to be ipoperable, but posstbly that scas deviation frow noswel operatios coaditions wa found.

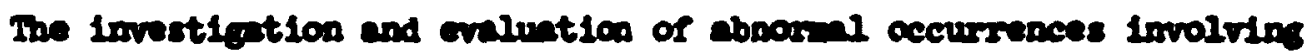
amubers were accoplisbed by searchise the literature for descriptions of manunctions of abubers in muclear sarvice, enelveing those incidents

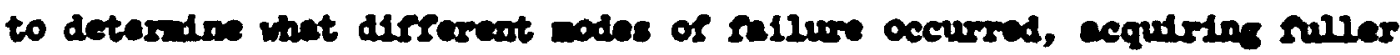
information about subber absornal beturior now reactor vadors and operetors and smbber monfecturars, breaking the list of spubbor mlowactions down into the varicus cetecorles of ebnormility, and rvaluating the altuation from the ovarier thas alned. 
The nethod eplojed to gatber the informtion about snubber fallures Is described under "docunantation." The nethod of categorizing the drsInctions is then explainod, and a table of reconded abnoral occurrences (cetegorized by cperator, reactor, date, rulure node, and numpucturer) is presented and sumarized. The results and conclusions of the investigations are than presented in a "Sumary" section.

The table of recorded aboorind occurrences along with the abstracts listed in Appendices A and B ahould provide a valuble reference for $\mathrm{N}$ ture investigations as well as docunent the major areas of concern regarding smubber perrousance.

\section{Docunentation}

A multiphasic docunentation effort was carried out (1) to assess the monitude of the problen of deficlent smubers in the melear-porer industry, (2) to obtain an orerview of the results rran RO Bulletins 73-3 and 73-4,* and (3) to deternine wai response the maclear-poser industry bas ade to the problen.

The first phase of the effort was a search of the AES (BRDA) dockets tron 1966 to 1975 . This was perforned by searching the computerized file of the luclear Safety Information Center at ORI und $r$ the ccmbined terms or "fallure" and "shock absorber" and of "failurp" and "support structure." The first cabination ylelded 131 references to items about fallures of shock absorbers, and the second produced 173 additional references to items about fullures of support structures not speciflcally referred to as "shock absorsers" in the indexing system. The great mafority of the pertinent references was generated by the use of the index term "shock absorbers." Better than got of the references thus found were of interest to and of use in the study. Less than $5 \%$ of the references generuted under "support structures" (other than shock absorbers) were of interest. The bibliography on shock-absorber fallures gererated by the NSIC search is reproduced bere as Appendix A.

- See Appendices C and D. 
The ISIC search was replicated and expanded to searching puclear Science Abstracts $\mathrm{from} 1964$ through 1975 using the Fircal asten based at ORul. The following index terns were selected for use in this senrch by noting the cross indexing used for pertinent articles turned up in a preLiminary run:

\begin{tabular}{|c|c|c|}
\hline $\begin{array}{l}\text { shock absorbers } \\
\text { seals } \\
\text { test ing } \\
\text { selsic efrects } \\
\text { kinetic energs }\end{array}$ & $\begin{array}{l}\text { railures } \\
\text { supports } \\
\text { reactor mintersince } \\
\text { i pact shock } \\
\text { shock } \\
\text { denping }\end{array}$ & $\begin{array}{l}\text { leats } \\
\text { specifications } \\
\text { earthquises } \\
\text { defects } \\
\text { restrints } \\
\text { inspection }\end{array}$ \\
\hline
\end{tabular}

Appropriate combinations of these terms (e.g., "seisnic erfects" and "dnping") were searched, and printcuts vere obtained wen the nuber of references generated seewed to indicate that such a search night be worthinile. The descriptions used and the respective nuber of citations generated are shown in Table 2. Again, the great majority of userul citations cane rrom searching under the index term "shock absorbers;" these are listed in Appendix B.

Engineering Irdex wes searched for the years 1973 and 2974 on the RRCOA systes. After a prelininary search, the file was searched under appropriate cosbinations of the terns:

$\begin{array}{lll}\text { sbock absorbers } & \text { inpection } & \text { shock wores } \\ \text { muclear reactors } & \text { insulntion } & \text { standards } \\ \text { muclear power plants } & \text { mintenance } & \text { stresses } \\ \text { earthquake erfects } & \text { piping systess } & \text { testins } \\ \text { eartbquake resistance } & \text { reliability } & \text { vibrations } \\ \text { failure } & \text { seals } & \text { velding }\end{array}$

Wo entries specifically about mulear-reactor shock absorbers were found in the open 1itereture by this seans.

The governent-report 11terature wes searched for the per1od 1964 to 1976 using the Govern ant Reports Anoouncenents flle capiled by IIIs on the DIfIOC systes at Iockheed in Palo Alto, Callfornis. Inis coarch printed out all citations with the doecriptice "ambber" (6), all iteas with the terms "shock absorber" or "shock absorters" combinod with "reactor" (15), all 1teas with "shock absorber" or "shock absorbex" conblocd with "muclear" (23), and all items with the descriptions "rwetor weinde 
Table 2. Huber of Cltations Generated by Searching Juclear Science Abstrects fren 1964 to 1970

\begin{tabular}{|c|c|}
\hline Description & Citations \\
\hline Shock absorbers & 31 \\
\hline Restra:ints & 7 \\
\hline Selsicic efrects plus daping & 33 \\
\hline Seals plus leaks & 119 \\
\hline Supports plus specifications & 48 \\
\hline Supports plus performance testing & 22 \\
\hline Trpact shock plus daping & 5 \\
\hline Seals plus performace testjag & 45 \\
\hline Seals plus rallures & 45 \\
\hline Seals plns defects & 13 \\
\hline Seals plus inspection & 27 \\
\hline Testing plus supports & 73 \\
\hline Danping plin; testing & $\mathfrak{H}$ \\
\hline Kinetic eneror plus daping & 3 \\
\hline Supports plus defects & 11 \\
\hline Supports plus inspection & 21 \\
\hline Inpact sbock plus dempiog & 5 \\
\hline Restraints plus specifications & 1 \\
\hline Restruints plus inspection & 1 \\
\hline
\end{tabular}

effects" or "power plants seisulc effects." A total of 120 citations were generated, Including several citation to the docket literature and to the non-NEC (-ERMA) Goverbnent-report literature that had not been picked up provicusly.

The achine searches were suppleanted wth a mamal search of the Indices to the docket literature. Severel iteme were uncovered by that neans; wo nention of smbbers or shock absosbers we fo: ind in the printed indices prior to 1972. 
A review of the abstracts generuted by these searches led to the selection and inspection of some 150 docket reports on snutber nilures in ruclear reactors. These reports provided the besic information for the sumary table or raulty snubbers presented later in this report.

The last phase of the initial documentation effort was couprised of writing letters to the various reactor operators who were identified in the docket literature as having had probleas with smubbers. These letters asked if the operetors bad perforwed ang tests or evaluations or han received aw tast results on smibbers from snubber manfacturers or imiependent inboratories. They also requested coples of any dita generated by such tests or inspections. A copy of a typical inquiry is included here ac Appendix F. The returns fram these inquiries often explained more rully the mode and cause of in-service insufficiencies of smubbers, pointed to possible solutions to problems with smubbers, and/or led to additionel Iiterature avallable about the subject.

Follow-up contacts with snubber mamucturers, regulatory personnel, reactor operators, reactor desigeers, and smubber-component supplfers prorided information about smubbers, their design, and their use that had not been supplied by the frevious documentation efforts.

\section{Abnormelity Analress}

The smibber malfunctions reported in the docket literature were studIed extensively, and a schene of categorizing then was developai. Twelve categories were found to be needed to typify all of the fallures reported. Bach category was assigred a letter designation; those destgations and the fuults they stand for are as follows:

A. Hodralle-smbber reservolr low or enty because of lealy seass

B. Hydreulic-smbber reservolx low or cupty (cause undoternibed)

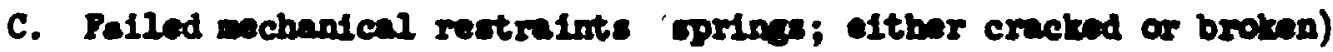

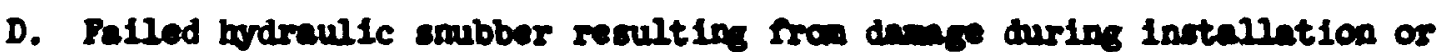
subsequent construction

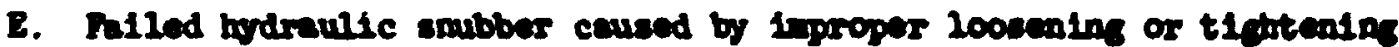
of attachant lockmuts and mbeequant smabber dislocation during installation 
F. Hechonical allunction (1.e., bent piston, feiled rod end, etc.)

G. Imbility of hydraulic smuber to nuction properly because of systemattechnent failure (pipe clarip, etc.)

H. Hudrulic-smuber performace did not watch service requirenents

I. Hodraulic-smubber perfornance not acceptable (1.e., inappropriate locknp rates, bleed retes, etc.)

J. Improper reassembly or installation (after ca-site disasse-bly and/or rebuizing)

K. Sembber dinensional specifications found out of tolerance

L. Contanination of modraulic-smuber Nuid.

The results of the andysis of smuber abnornal occurrences are given in Table 3. The nuber of leficient smubers is listed under each faultcategory letter designation. When the sctual number of srubbers involved was not specified, the symbol "?" was entered. Docket reports that found no fallures or that consisted of ABC-issued RO bulletins are noted as such. The products of only tro manfucturers have been specifically studied inservice and therefore account for all of the reported failures. They are Doted in Table 2 as mamueturers A i IIT-Grinnell) and B (Bergen-Petter8on). The last calum in the table provides a reference to the abstract of the docket report as listed in Appendix A. The deficiency totals are sumarized in Table 4.

\section{Sungy}

In this section, each fault category is considered separately with ccunents from the princlpal investigator and selected experts ramiliar with the smaber problea.

\section{Cate ory A. Hydrulic-anubber recervolr low or cuty because of leaky} ecels $(513$ cases)

This category, by far, 1llustrates the wost cemon problem with hydraule smbbers. he described ariler, ovidence of learnge of hydrulic Fuld dwe to seal fallure proupted Po Bulietins 73-3 and 73-4 wich Inotigated the Bergen-Patterson investigation of seal materials. It was 


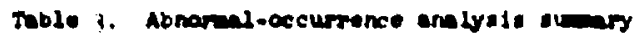

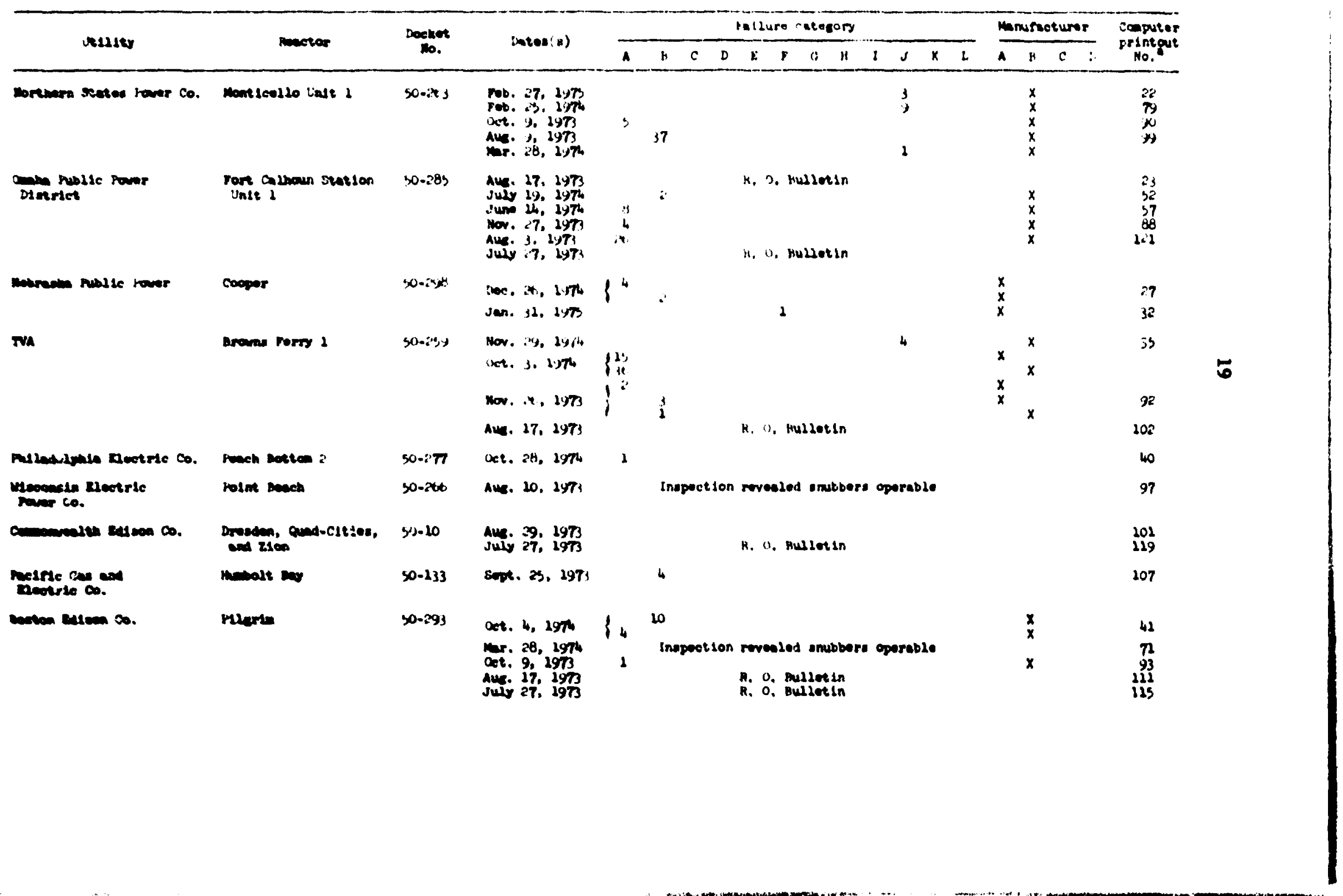




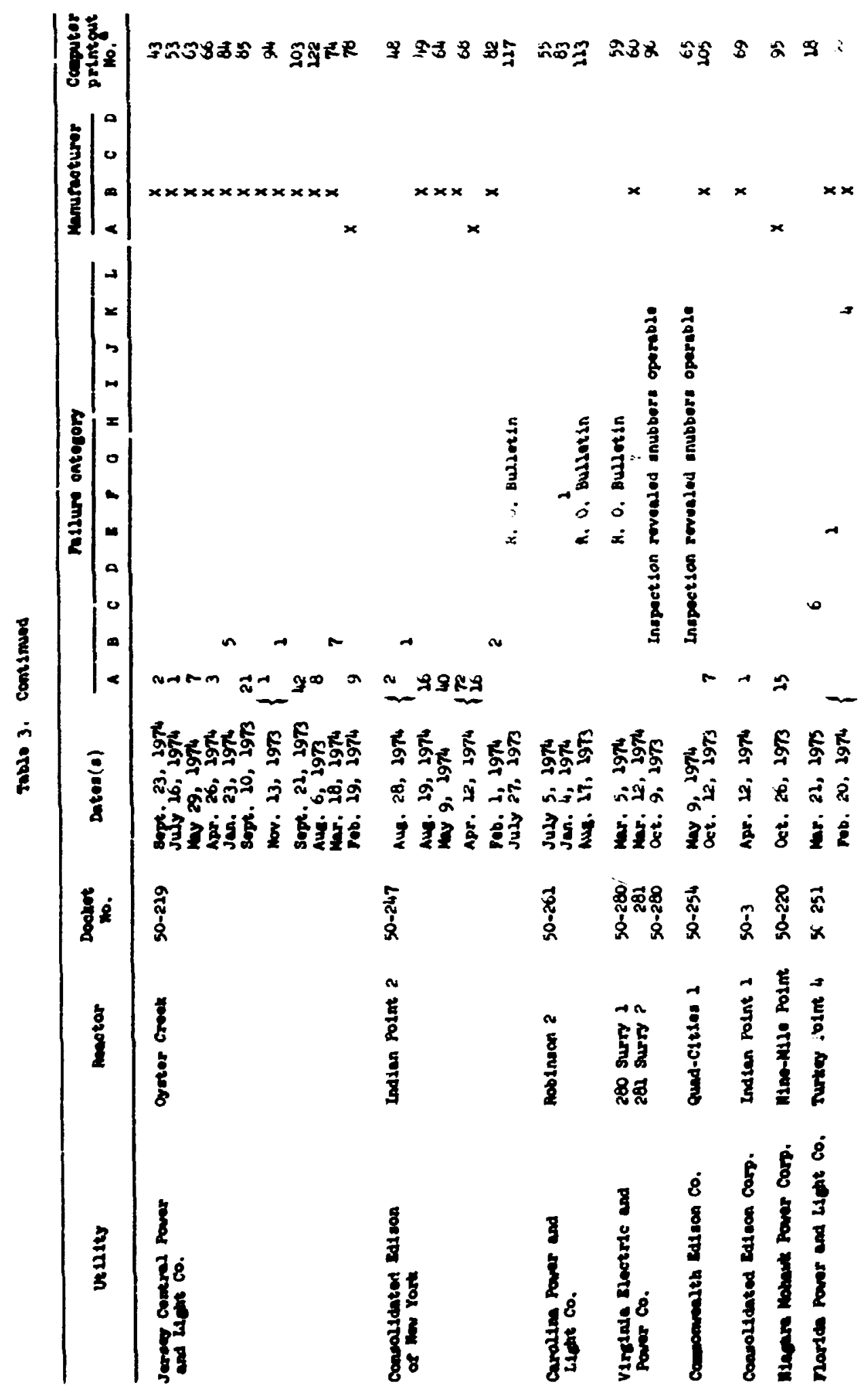


Table 3. Continued

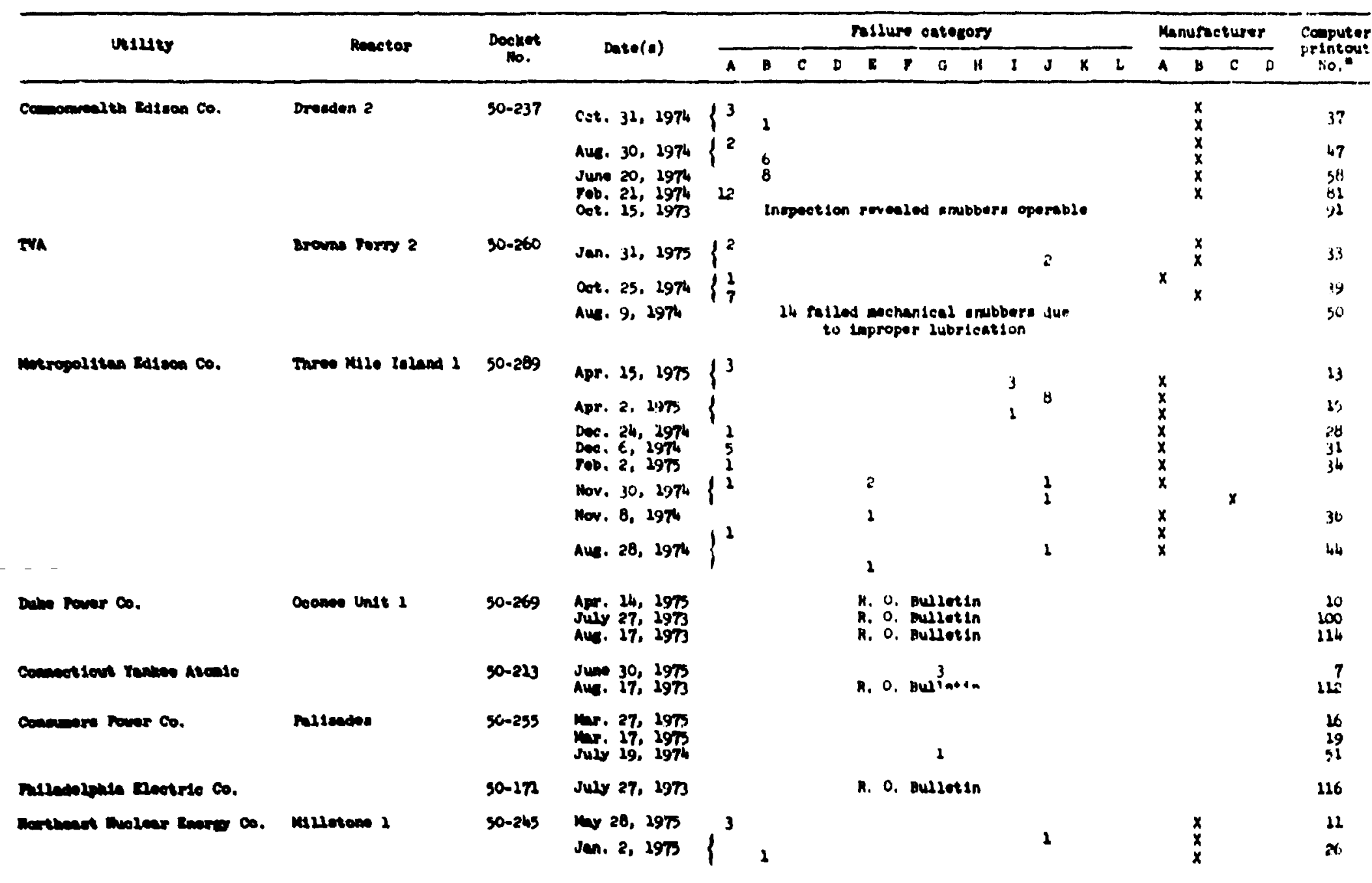


Table 3. Cont inugd

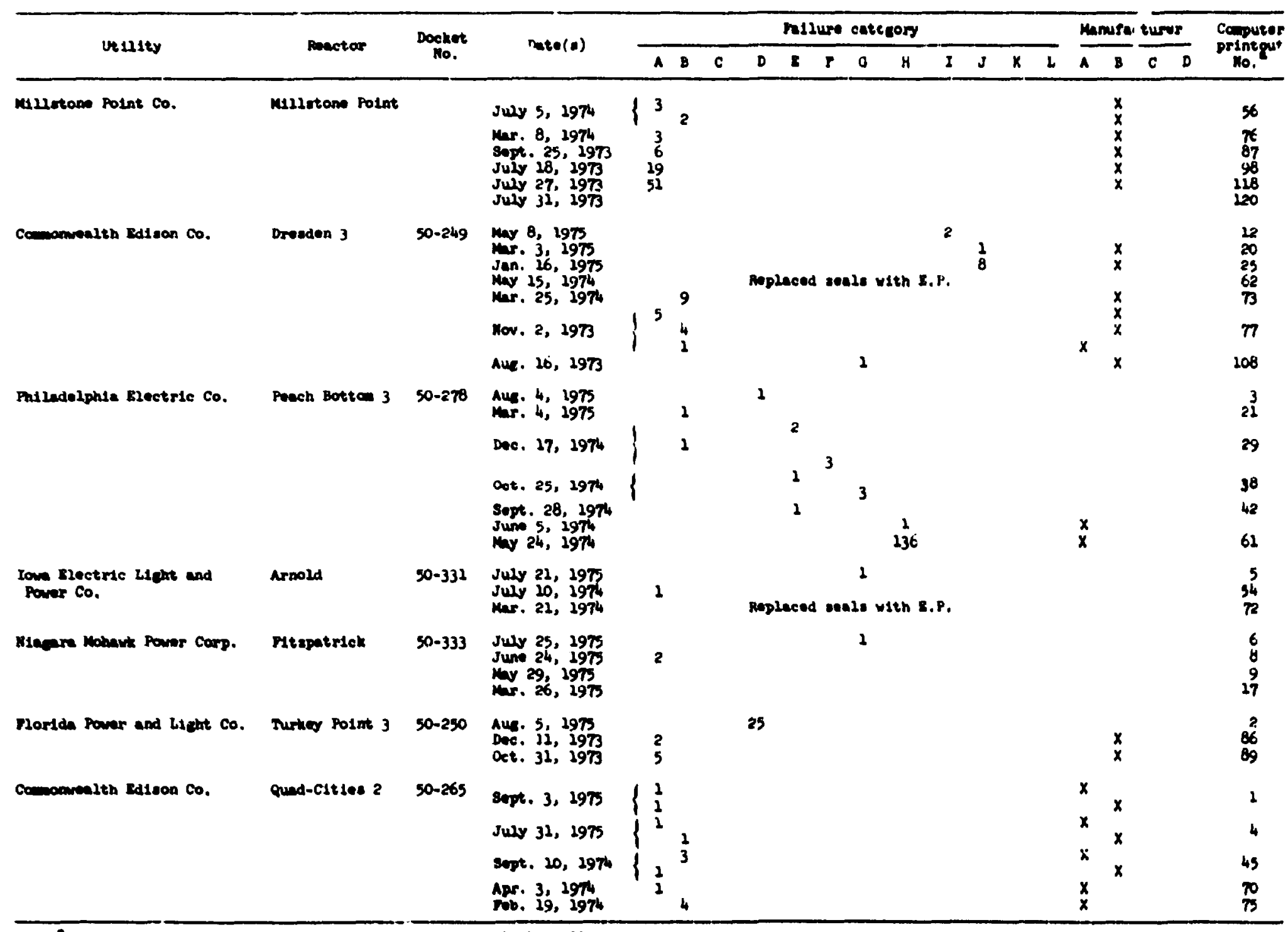

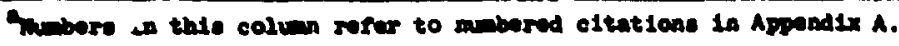


Table 4. Fallure-mode compliation

\begin{tabular}{|c|c|c|c|c|c|c|c|c|c|c|c|c|c|c|c|c|c|}
\hline \multicolumn{13}{|c|}{ Thllure categories } & & & & & \multirow{3}{*}{$\begin{array}{l}\text { Totel No. } \\
\text { of Iollures }\end{array}$} \\
\hline \multicolumn{13}{|c|}{ No. of rallures } & \multicolumn{4}{|c|}{ Manufacturers } & \\
\hline A & $\mathbf{B}$ & $\mathbf{C}$ & $D$ & $\mathbf{E}$ & $\mathbf{s}$ & $\mathbf{G}$ & $\mathbf{H}$ & $\mathbf{I}$ & $\mathbf{J}$ & $\mathbf{K}$ & I & Other ${ }^{b}$ & A & $\mathbf{B}$ & $\mathbf{c}$ & Unkniown & \\
\hline .23 & 117 & 6 & 26 & 9 & 5 & 10 & 137 & 6 & 40 & 4 & $?$ & 14 & 242 & $56 ?$ & 1 & 82 & 887 \\
\hline
\end{tabular}

A, Grinnell; B, Bergen-Patterson; C, McDowell Wellman.

bimproper lubrication. 
determined in this report that polyurethanes were not coupatible with service conditions or with the hydraxlic fluid. It was recomended that ethylene propylene (EP) materials, such as Parker E652-90 or E529-65, be substituted, and for eren greater life expectancy, Parker E740-70 or Stillman 57-723-70 should be used. Units used in radiation areas will be charged with GE 1154 Pliid and those in nonradiation areas will be charged witii GE SF 96 (200). Also, it was determined in the Bergen-Patterson report that the maximum permisssble radiation dosage for the EP seals is $10^{7}$ rads at room temperature.

The polvurethane-EP scel-replacement program has been going on since November 1973, and therefore selected smubbers with EP seels have seen a maximum of approximately 2 years service. Although a great number of snubbers havi been changea to EP seals, it is not clear at tijs time wrat percentage of smubbers still contain polyurethane materials. At this point, the authors have not discovered one incident of loss of hydraulic flutd tue to seal degradation in smbbers fitted with EP seals. Hovever, fluid has been found leaking out of smubbers fitted with EP seals for other reasons. These occurrences will be discussed in more detail under categories $J$ and $K$.

Although the EP seals seem to be performing adequately from a material standpoint, it is important to consider the following:

1. Leta from servici-condition performance of snubbers with EP seals ar z incomplet from tise staxipoint of longevity related to failure due to wear and environmental effects.

2. The relationship between EP-seal degradation and radiation coupled with other environmental conditions has not clearly been established. A quotation fram the Bergen-Patterson report ${ }^{4}$ reaffirms this statement:

Hote that on: of the tests reported here shows the effects of radiation, while the other shows the effects of temperature and fluid. We have not tested the combined effect of all three, but we anticipate that the $108 \mathrm{~s}$ in physical properties may be greater than a simple addition of the seperate effects. 
Categary B. Hrdreulic-smubber reservoir lou or expty (caused undetermined)(117 cases)

It would seem that many smbibers in this category were leaking fluid due to raulty seals. But a lack of a formal evaluation precedure and resulting documentation obscured the reilure mode. Hevertheless, there were a mubar of lon reservoirs that definitely could not be attributed to seal fallure and resulting leabage. These low reservoirs can be attributed to the following: improper filling at the factory, leakage of fluid during shipping, and/or ixproper filling at the installation site. Improper filling includes incosplete bleeding of the snubber cyliader and reservoir to eliminte the pussibility of afr being trapped in the system.

Catepory C. Fafled mechanical restraints (springs; either cracked or broken) (6 cases)

There was only one location where this occurred (Turkey Point 4). The cause of fallure was not determined, but the occurrence doer indicate a possible need for inspection of spring restraints and for a study of performance requirements.

Category D. Failed hydraulic smubber resulting from damage durirs installation or subsequent construction (26 cases)

Failures in this category cannot be attributed to and particular cause since they are largely accidental and random in nature. But the chance of smubber damage due to construction activities can be minimized by the folloring considerations.

1. Special care should be taken by the utilities to install the smubbers at the best time to minimize subsequent construction damage.

2. A number of smubbers were rendered inoperable by damage to the extended piston rod. The surface finish of the piston rod is important to eliminate seal damage, and presently there is no protective coverins for this part of the smubber. Special care in handing the snubber is indicated. 
Caterory B. Fuiled hydrulic smuber caused by ilproper loosening or tightening of attachnent loclonts and subsequent snibber dislocation during instefiation $(9$ ceses $)$

There exists a definite problem with smbbers located in areas that experience high-frequency system vibratisi:. Although the installation instructions do not recomend the use of any materials or devices for the prevention of loosening of the bolts by vibretion, it would seen that scine method should be employed to ensure that such loosening vill not occur.

Caterory F. Mechanical mal nunction (1.e., bent piston rod, foiled rod end, etc.) (5 cases)

The mumber of failures in this category was sall, and they can probably be attributed to inproper installation or lack of proper deslem analysis by the utility in regard to thermal expansion and subsequent smubber loading.

Category G. Inubility of hrdraulc smubbers to nuction properly because of system-attachment failure (pipe clome, etc.) 10 cases)

Although this investigation is primarily concerned with the proper operation of the smubber itself, the performance of the associnted attachment mechanism is certainly crucial. It seems that the smubber mamiracturers leave the design and installation of the attaching wechnism to the utility. Since failures of attuching mechanisms bave been noted, there definitely needs to be a coordination of effort among the utilities, pipe-clamp manufacturers, and smbber manufacturers in order to produce a complete restraint system that will runction properly undar all types of service loadina.

Catezory H. Hydraulic-smubber performace did not match senvice requirements (137 cases)

A thorough investigation of the 136 smbbers at Peach Bottcm 3 whose performance did sot match service requirenents revealed that the bleed rate was not fast enough. An adjustment of the bleed oriflce eliminated the problem. One smubber's bleed orifice was closed. 


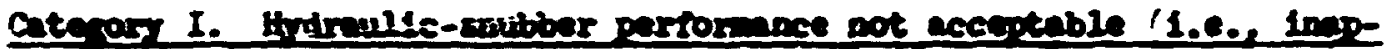

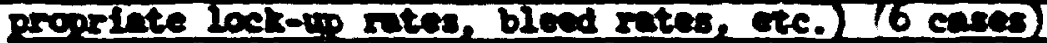

The Three Mlle Island locldeat lastlented 30 Bullet in 75-05. Carenul ovaluetion of the subbers iavolved servaled that $20 s 8$ of lock-up ablilty could be attributed to contaninats in the poloulic nuld building up on the poppet-valve oprings. This incident ar well be entegorized

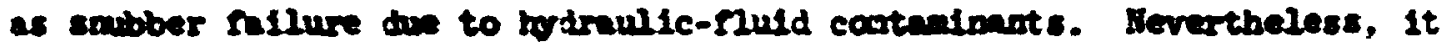
we felt that the requeste for performace evalunt ton of subbere as required by 80 75-05 are rarreated and that sigaiflcant accumulation of date of this anture is acesesary.

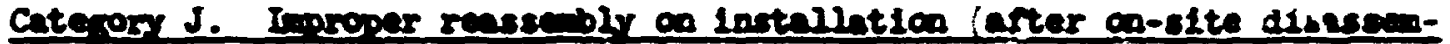
bly ad or rebullidin? 140 cases:

The changing of smibber seals rrou polvurethane to EF revealed a masber of problens with send-replncewent procedu-r: and seal desion. Certain 0-ring replacenents are almost impossible to pes farm in the field witbout

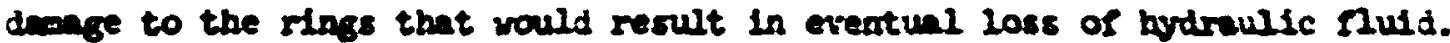
These problems can best bi described by personnel funiliar with the sealroplacsont procetures tbat have actunlly attoupted to rebulld a smobber. Their coments vere recelved in response to the questlonnalre sent ourt to reactor operators Identified in the tocket. Ifterature.

The epparont cause of 1088 of ofl in the three Bergen-Patterson arrestors is twofold. The errestor an the IfIR gyoten outside the dryvell had an unscceptable low anount of oll beceuse the inner lip of the piston rod packeng ring we torn. The packing ring contalos an 0-ring inside a U-cup. This O-ring stiffens the packing ring to anch a degree thet during the Installation and oparetion of the packing ring, the Inner $21 \mathrm{p}$ of the U-cup 1s subjest to tearing pernittine ofl to 2eak out of the arrestor unit. The packing ring must be installed blind, preventing de-

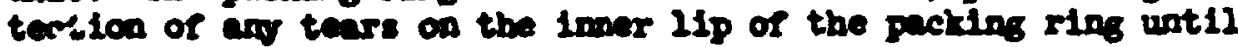
the arrestor unst is pliced in scrvice. - IVA

The piston rod end is a U-cup seal with a greader piece inented into the cup. During tbe rebullding effort, it was noticed that extrese eare had to be exercised in inctellation of this seal to provent the spreader plece from becoulns separated tro the min cup seel. Wost of the leaking soubbers found on the eirat inopection after robuliding ware found to here pleton rod seals that had the spreader plece eqparated Inom the U-cup.

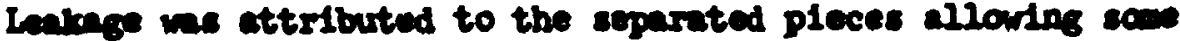


deformation of the U-cup seal dw of asserbly of the smubber, and thas loss or surficient sealing erfectiveness to allor slow leakage. - Moridn Power and Light

Caterary K. Snubber dinensional specifications found out of tolerance (L cesses

Although there was only one reported incident in this category $2: 3$ It Involves caly four smibbers, it should be noted that no other investifations of dimensional speciflcations are known to have been performed by the utilities. As a result of this, there ny be a need for a moreconprehensive investigation of design practices related to dimensianal specifications and tolerances.

Caterory L. Contanination of hydreulic-smuber Mluid (7 cases?)

There were two reported instances involving contaminated hydraulic 1uid. It is not evident at this point whether the forelgn material was Introduced at the installation site or was present in the smubber before shirwent. In one reported case (Three Mile Island 1) the fluid contaminants rendered the smubers inoperable, and the incident was categorized under fallure category I.

\section{COATHISS AID CONCLUSIONS}

Although it is premature at this point to make specific recomendations regarding smubber eveluation, a muber of important points need to be recognized.

1. There is insurficient information regarding EP-seal integrity under the cambined effects of radiation, temperature, and wear.

2. Service data on EP seals are incomplete at this time.

3. Proper fill-and-bleed procedures have not been described clearly to the utilities. Fossibly, there is a need for a personnel-training progran in this area. Qunity control in regard to air trepped in hyiraulic cylinders charged at the ructory should be reviewed.

4. There may be a need for an inspection-8urveillance program of mecharical restraints (oprings). 
5. Indequate protextion is prorided for the extended sumber piston rod.

6. Bolt loosening of subbers seeing sifoirficant system vibretion is a derinite proble.

7. It is felt that stress analysis of smubber componests, as nifit be described in ASIE, Sect. III, Subsection IF, will reveal nost design probleas related to coponent over-stress.

8. Coordination anong the utilities, the pipe-clem and pipe-banger mamucturers, and the snubber moufecturers is iportant in order to assure an adequate attachnent nethod for the smobber.

9. Swubber specifications, as prescribed by the utilities, ny need to be reviewed more carefully. (This topic will be dealt with in detail in a ruture report.)

10. Date on swubber performance related to lock-up velocity and seal integrity should be accumlated on units that are in service.

11. Ethrlene-propylene-seal installation procechures need to be reviewed and improved by the smubber mamucturers.

12. Ethylene-propylene-seal desifon should be evaluated for wear in smubbers that experience system vibration.

13. Qusity control of smubber dimensional and performace specificatior: and inspection should be revierred.

14. Contaminated hrdrulic Fuid is a definite problea, and a careful review of all phases of control of fluid purity is necessary.

This investigation has attempted to piopolnt problea areas related to smubber performance. A subsequent report will be concerned with the adequacy of methods used by the reactor vendors and operators for determining smubber specifications.

\section{REFRRATCES}

1. Asare Boller and Pressure Vescel code, Sect. III, "huclear Pover rlant Components," American Society of Mechanteal Engineers, Hew Yort, 1974.

2. "Additional Materials for Cauponent-Supports Section III, Subsectios ITP Class 1, 2, 3, and MC Contruction," Case 1644-2 in Cases of 1890 Bolier and Preseure Vessel Coxde, American Society or Mechanical Englneers, New Yort, 1975. 


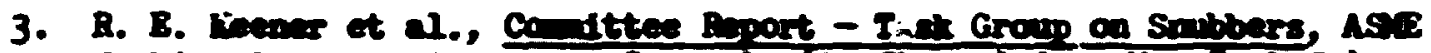
Worting Group on Componit Stgports (drant revision, Mo 8, 1975).

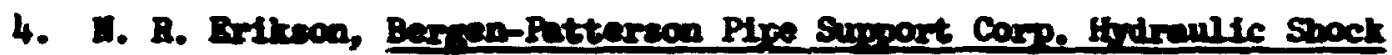

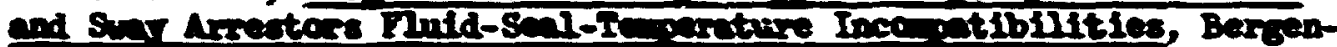

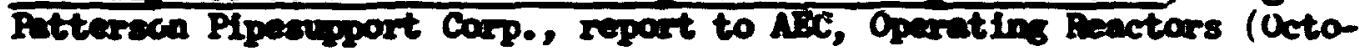
ber 7, 2974).

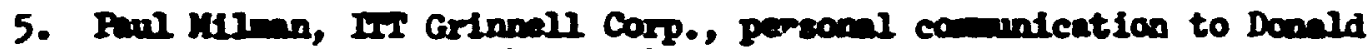
routh, Usuld (Apeil 14, 1975). 
APFEADICES 
APPENDIX A

NSIC BIBLIOGRAPHY OF FAILURE OF SHOCX ABSORBERS AND OTHER SUPPORT STRUCTURES (FAILURE ABSTRACTS) 


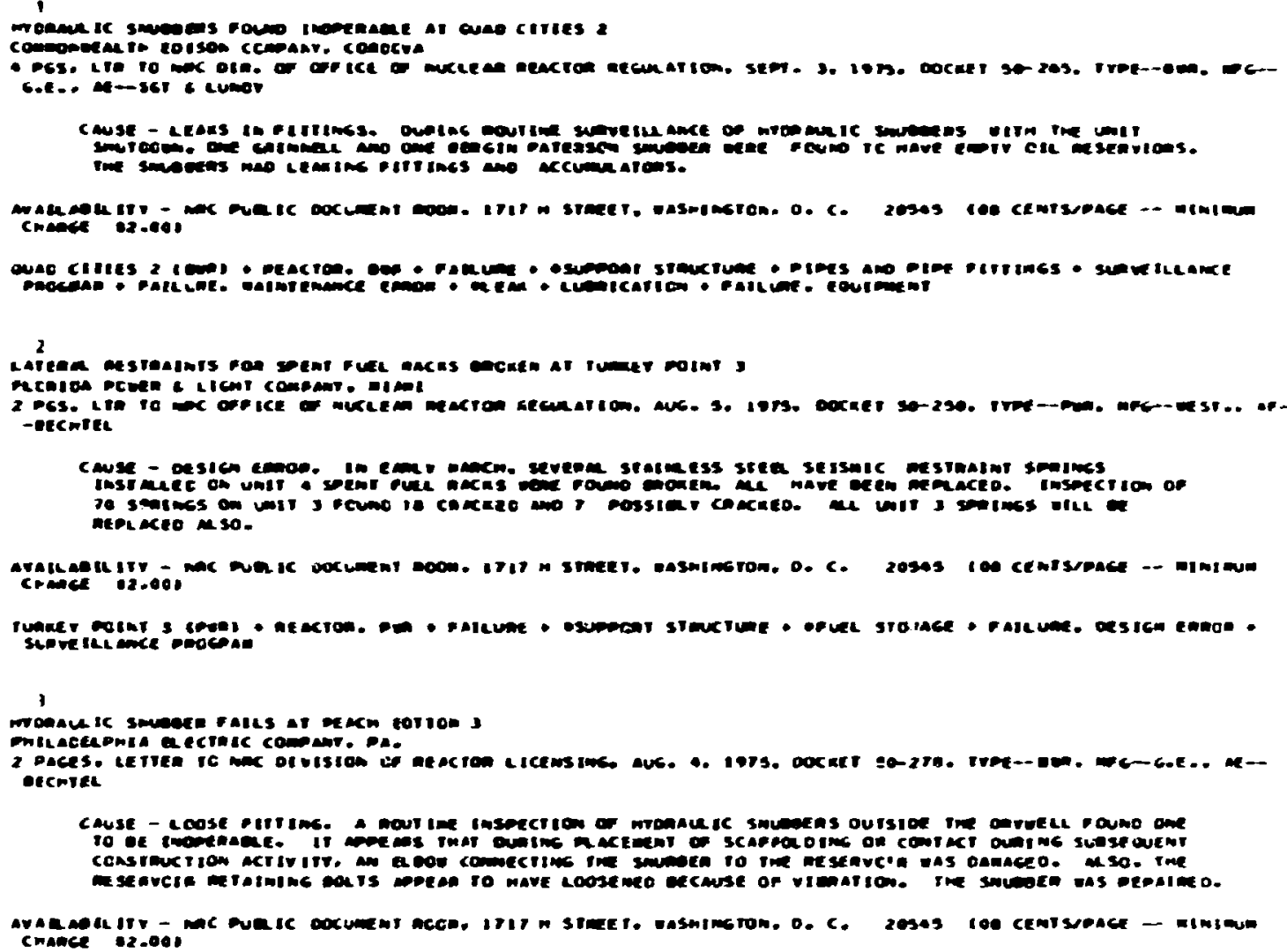

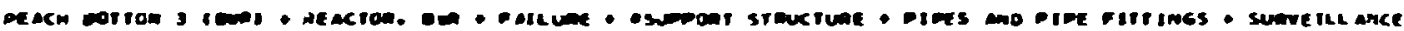

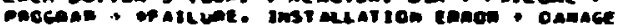

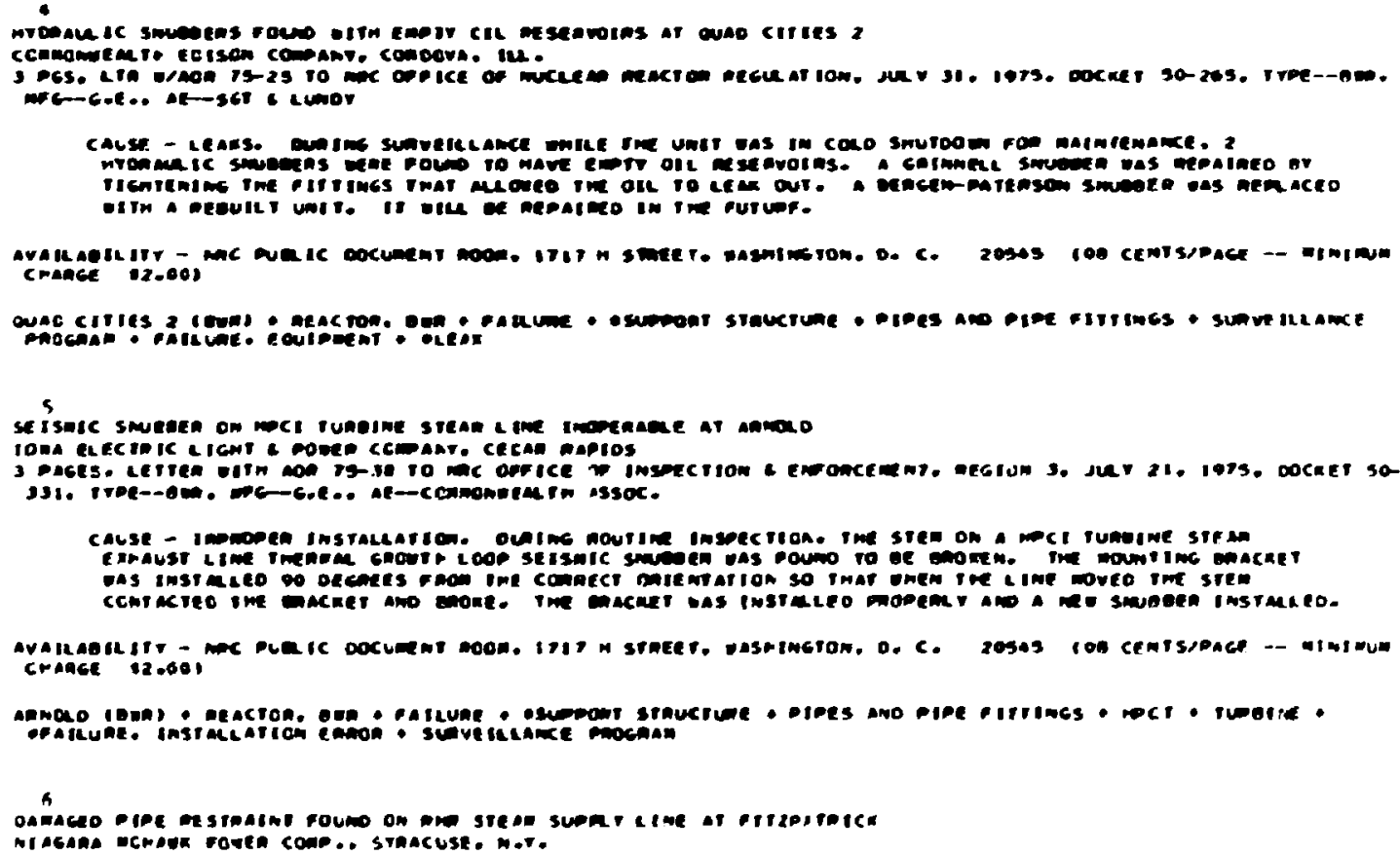




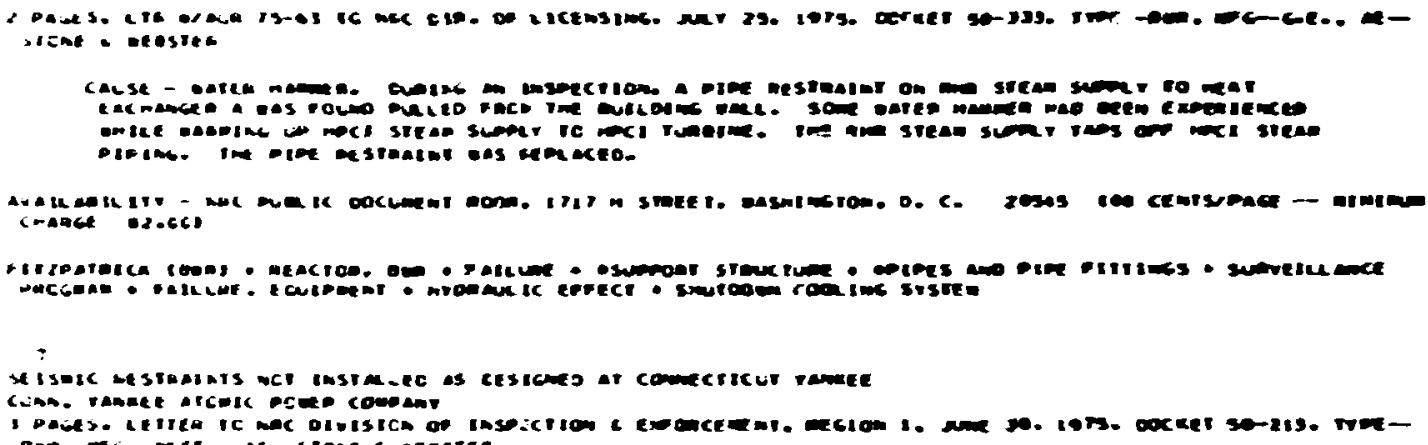

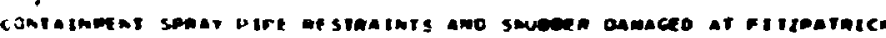

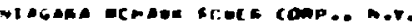

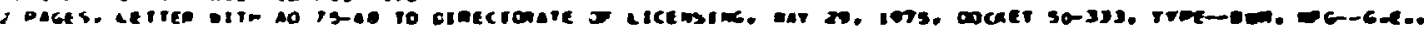
aE--stcae coreste.

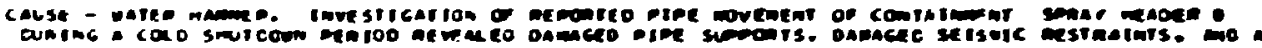

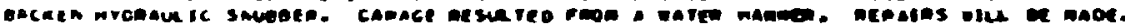

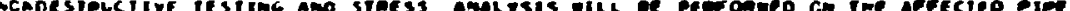

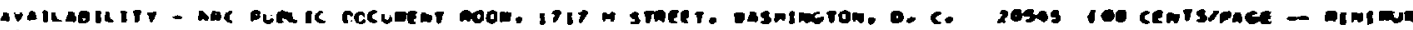
crance $18-c c t$

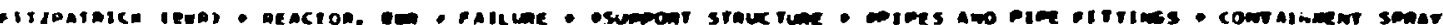

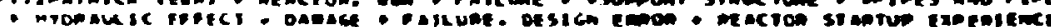

11

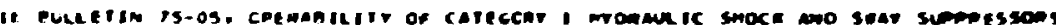

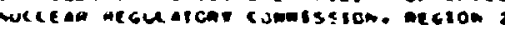

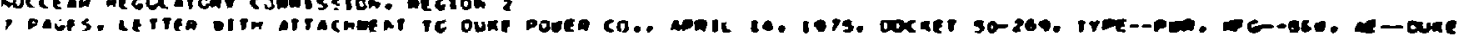

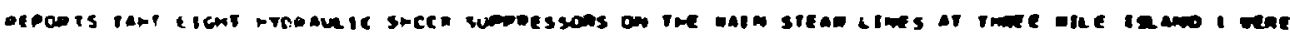

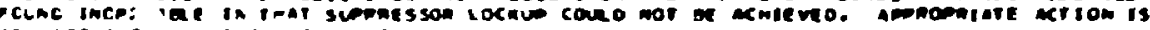

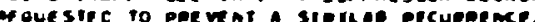

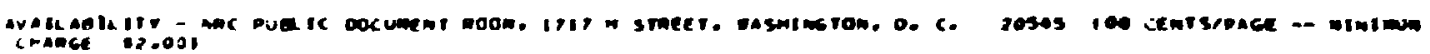

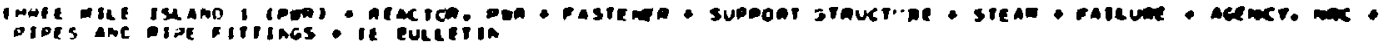

\footnotetext{
1

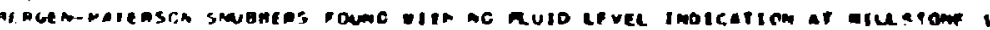

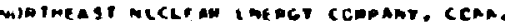




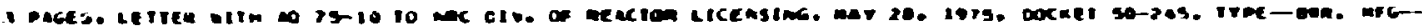
G.t. a-teascc

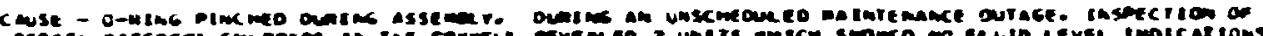

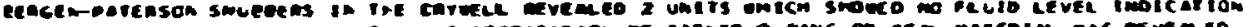

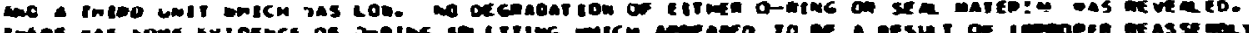

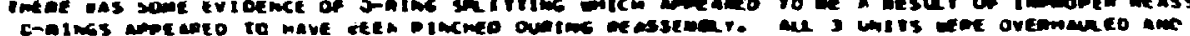
cinings atec.

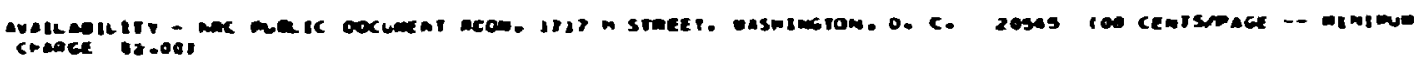

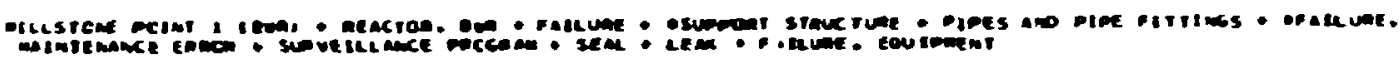

12

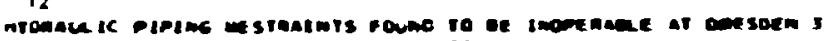

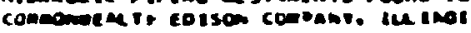

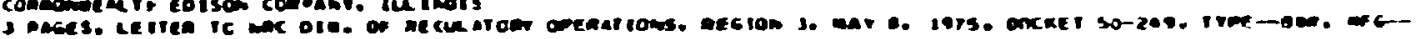
G.e.. el-16i c enov

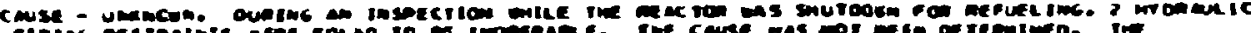

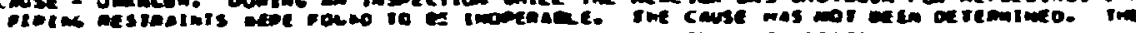

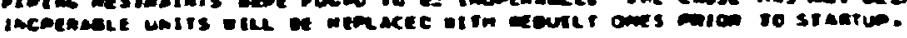

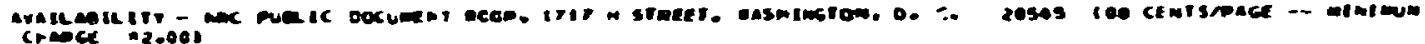

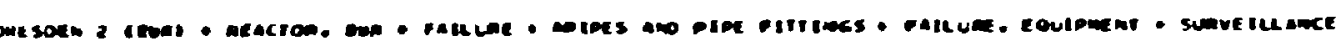

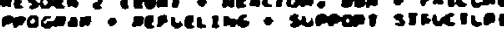

13

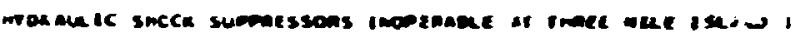

C rmopor iran colson comant. De.

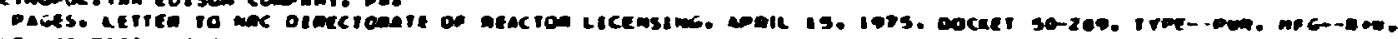
s=-citecer assce.

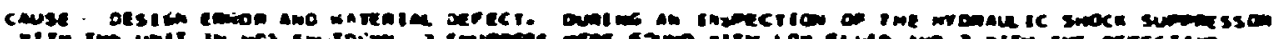

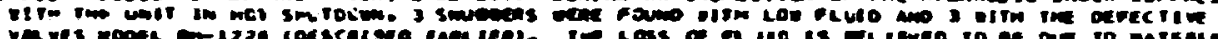

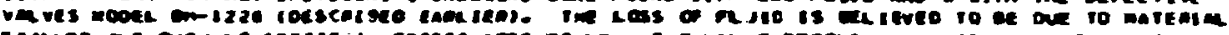

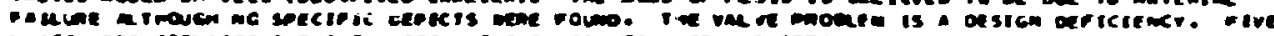

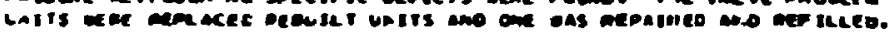

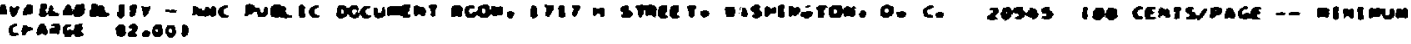

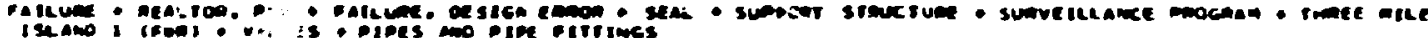

1.

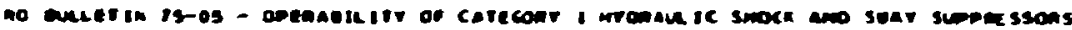

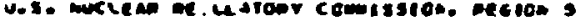

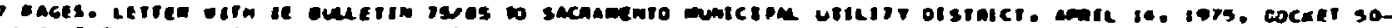

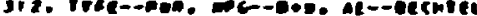

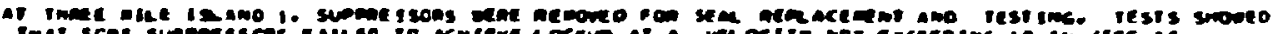

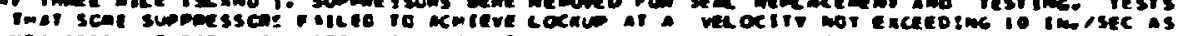

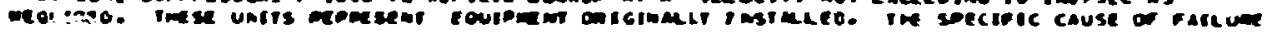

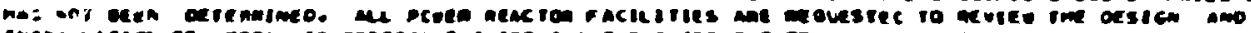

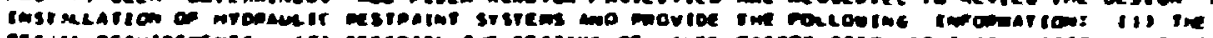

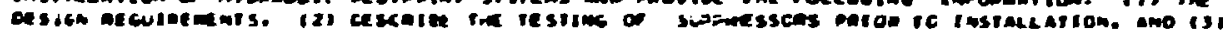

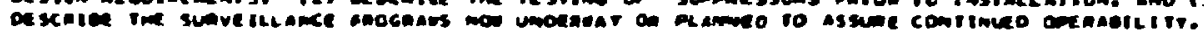

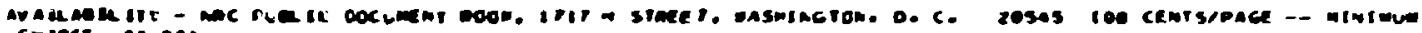
Cancest to.00:

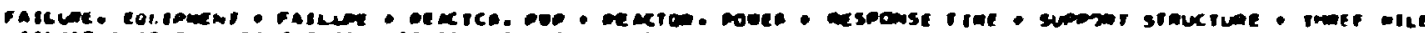

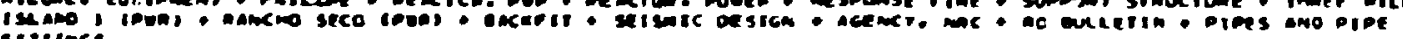
cotions

15

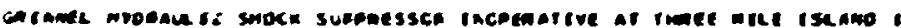

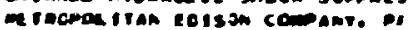

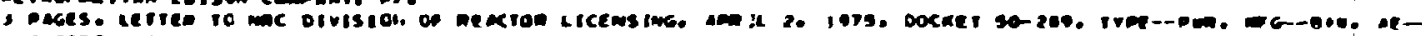
csuani assoc.

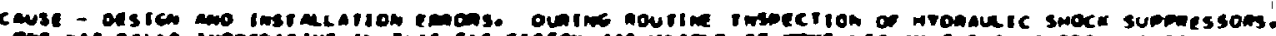

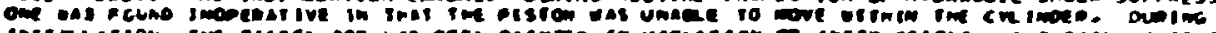

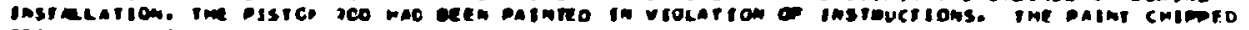

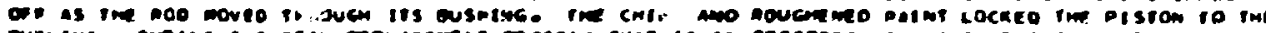

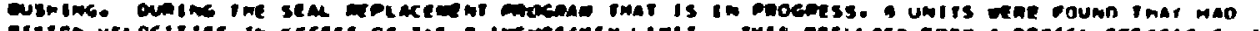

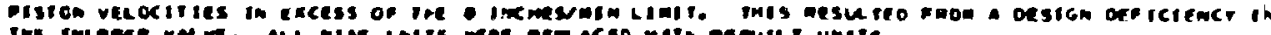

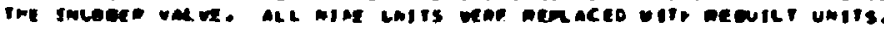

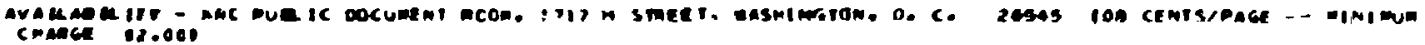




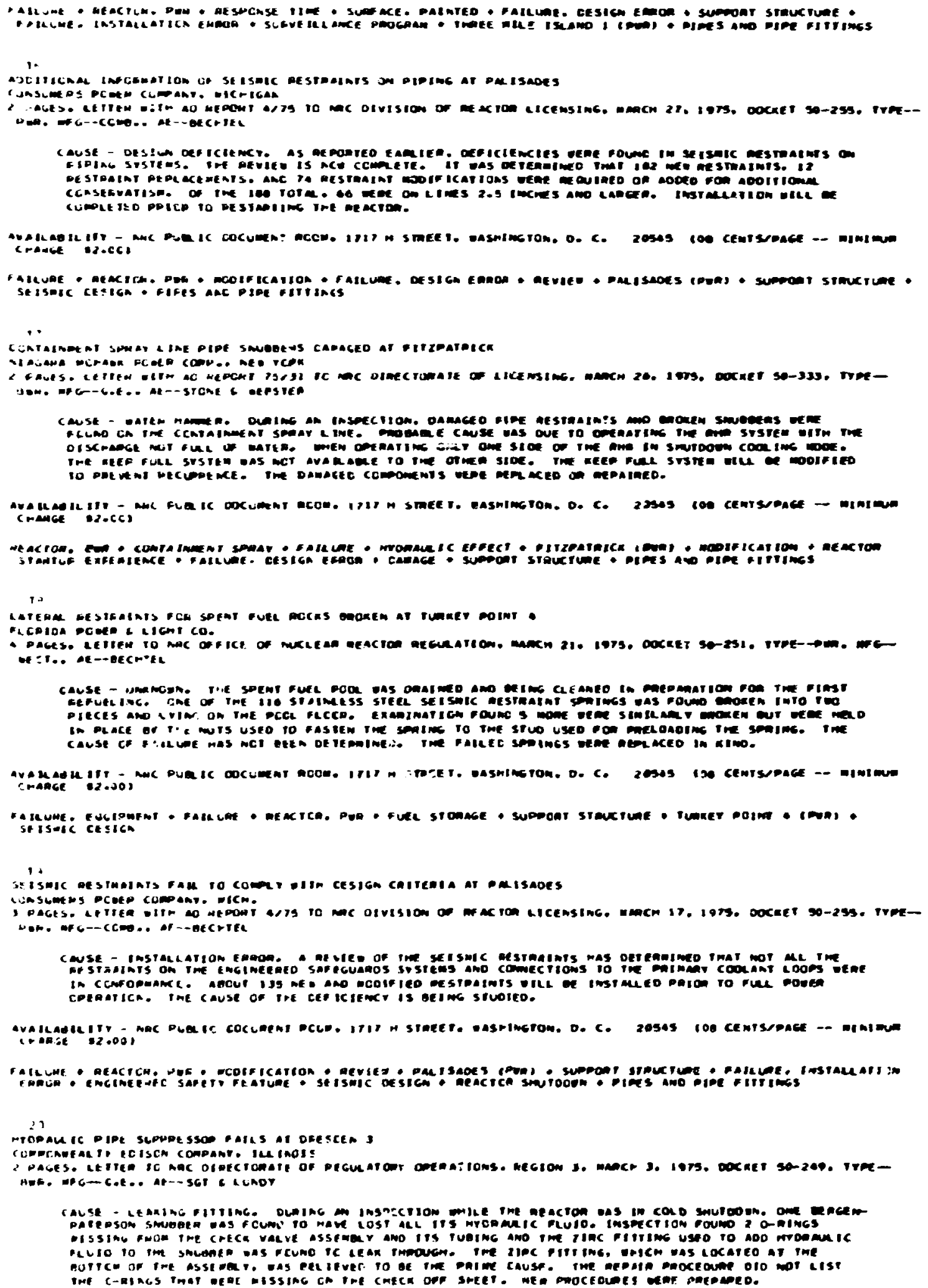




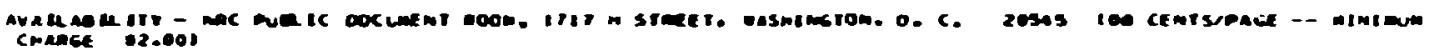

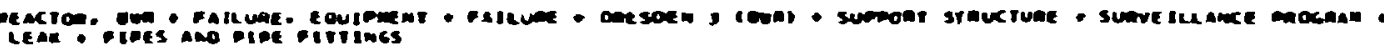

21

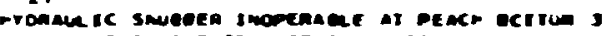

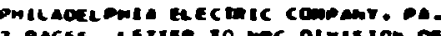

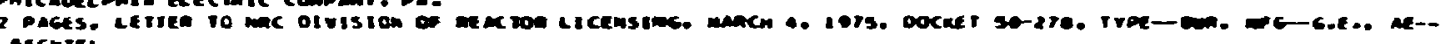
Becmiel

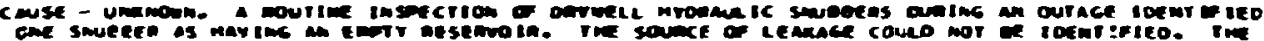

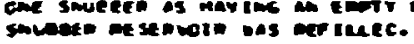

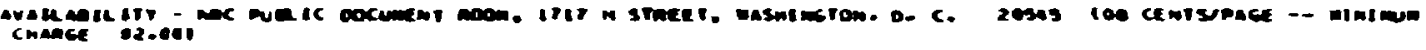

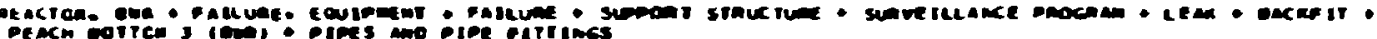

22

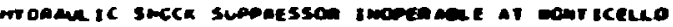

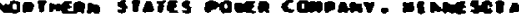

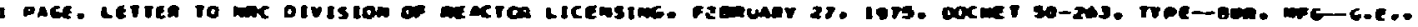
Af-ECAntes

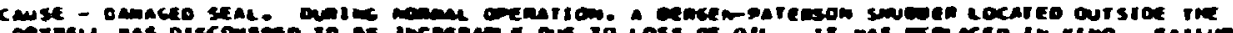

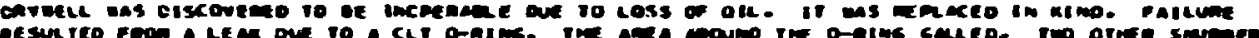

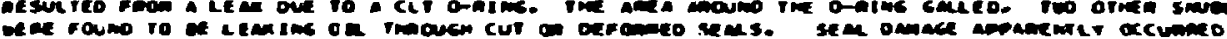

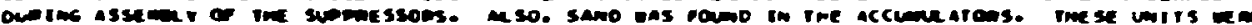

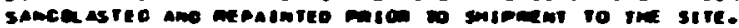

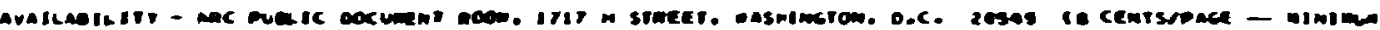
Crance ezees

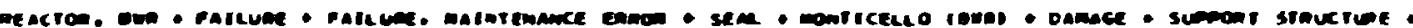

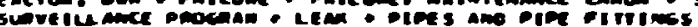

23

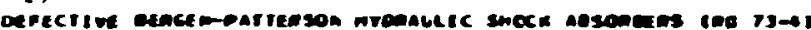

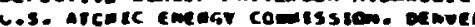

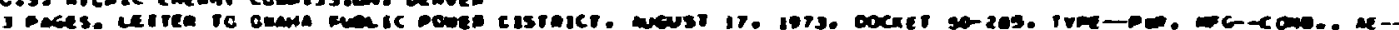
cioes riu

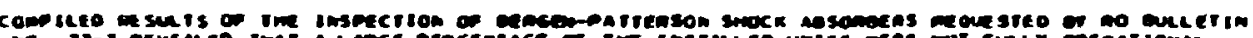
no. 73-3 atve ce

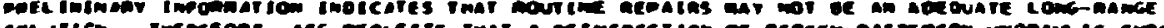
verenation -

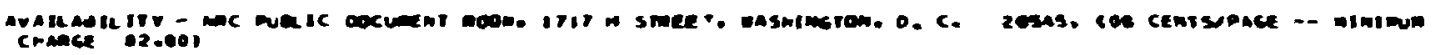

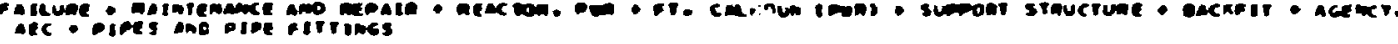

24

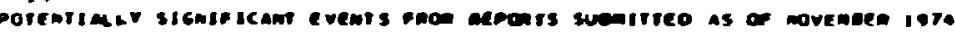

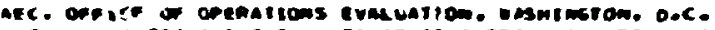

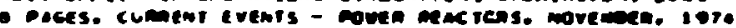

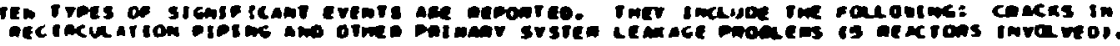

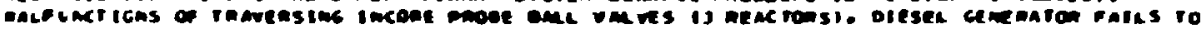

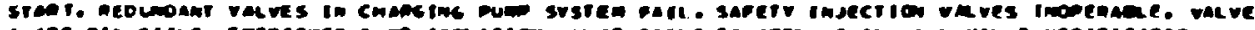

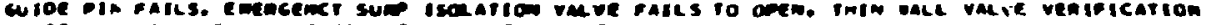

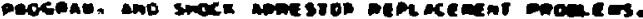

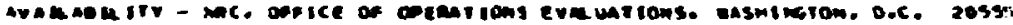

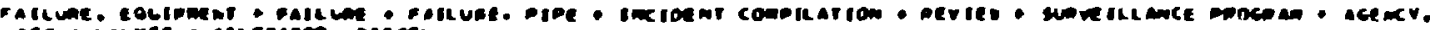

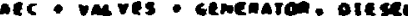

29

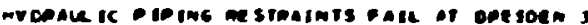

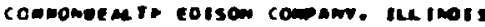

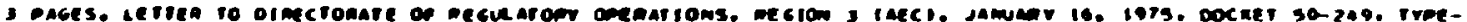

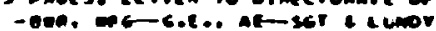

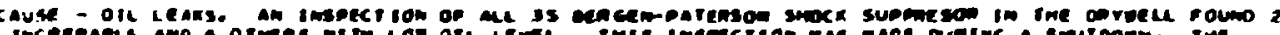

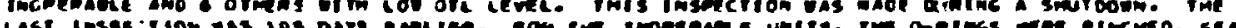

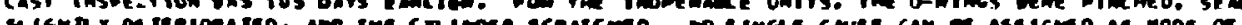




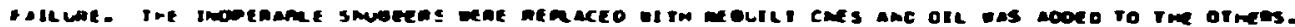

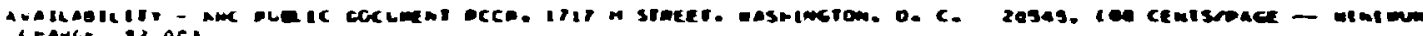

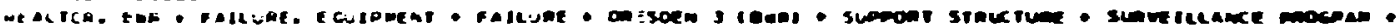

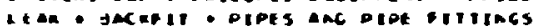

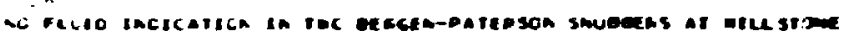

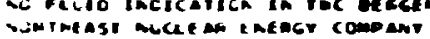

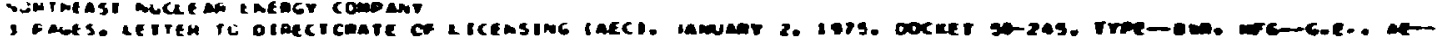
resce

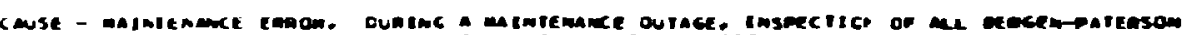

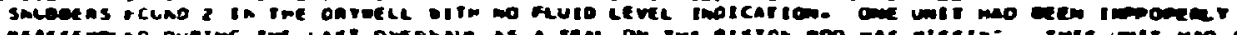

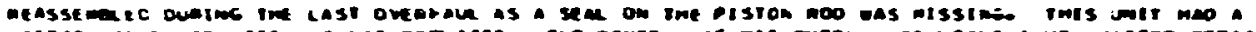

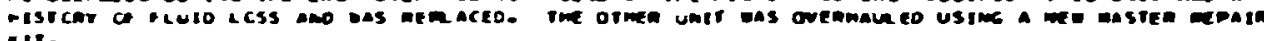
air.

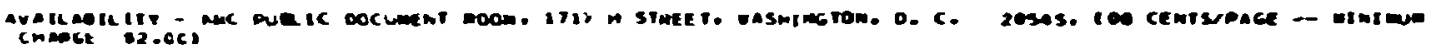

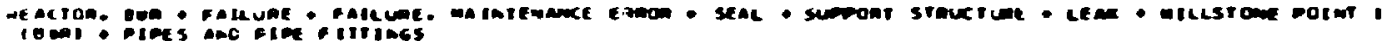

.

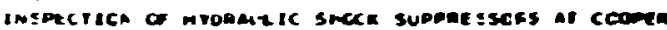

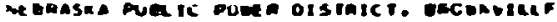

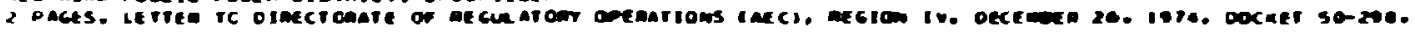

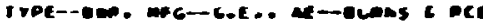

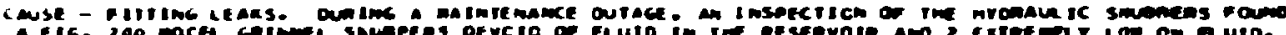
Fleic

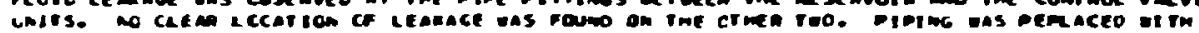

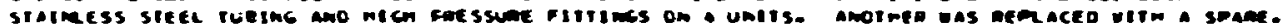

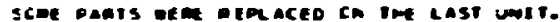

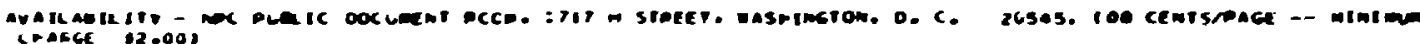

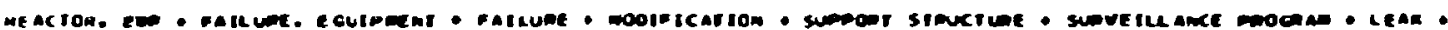

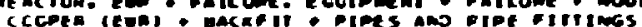

$\therefore$

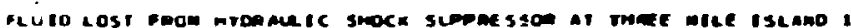

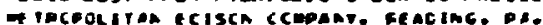

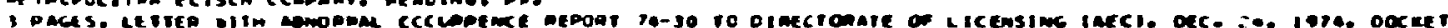

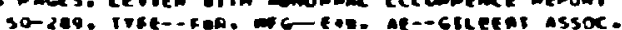

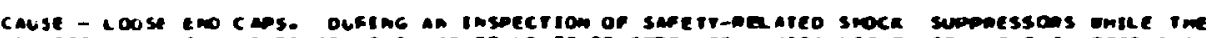

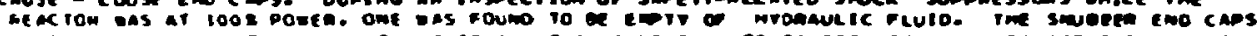

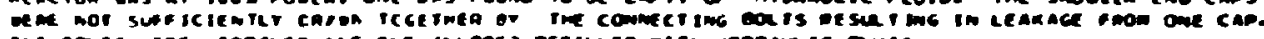

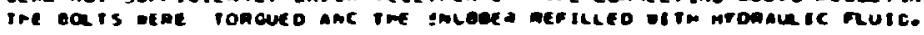

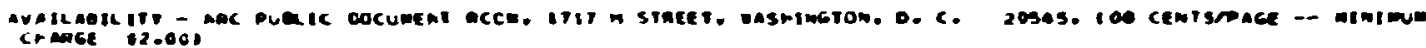

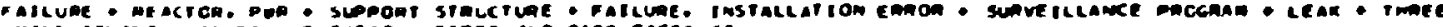

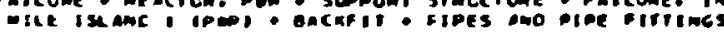

.

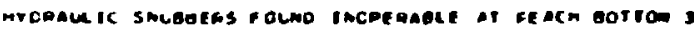

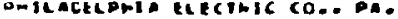

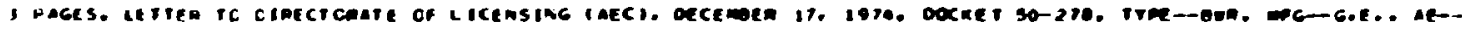
ancries

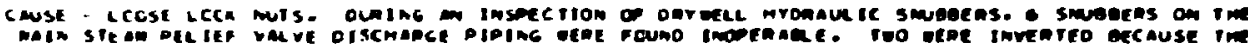

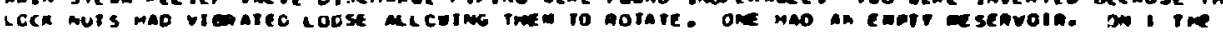

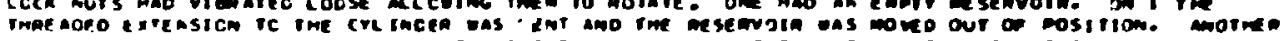

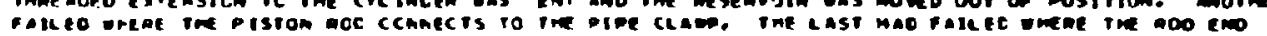

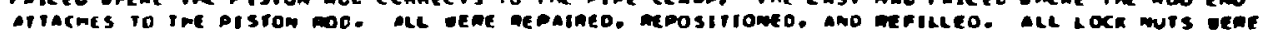
criaches id ine pistom

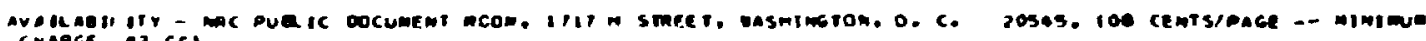
MARGE T.CE)

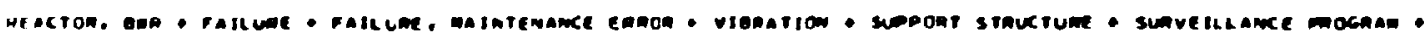

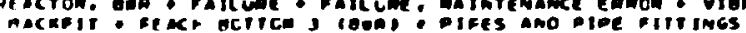

, 1

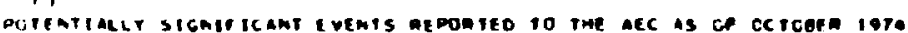

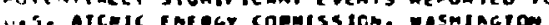

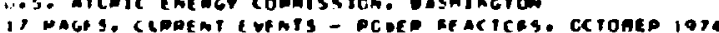




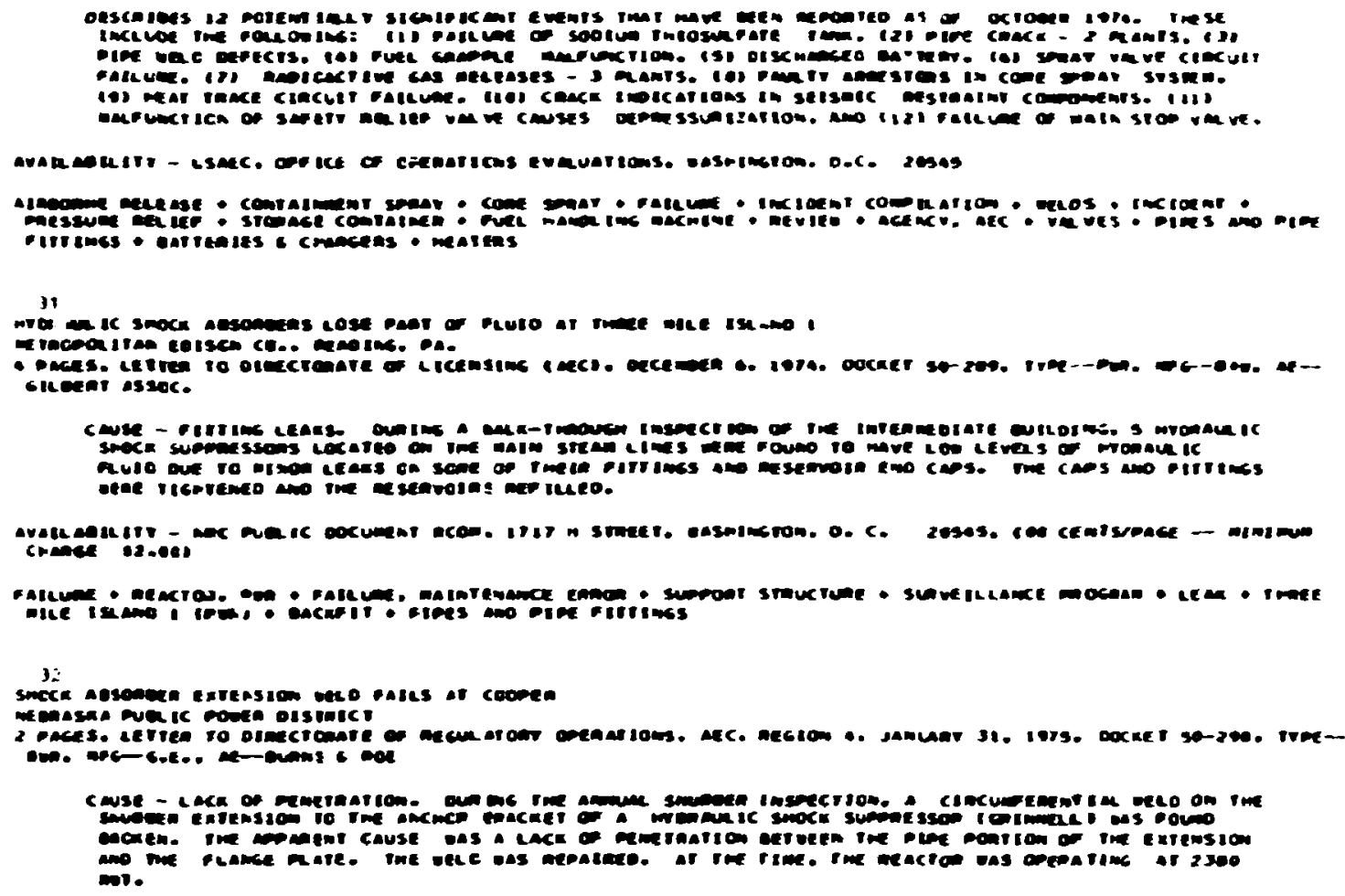

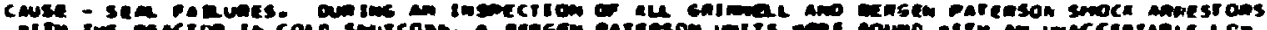

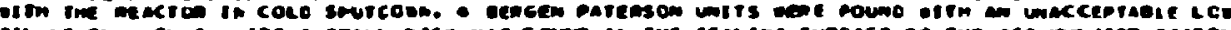

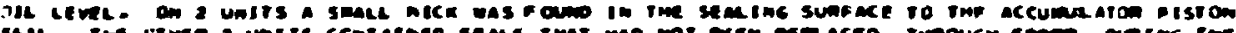

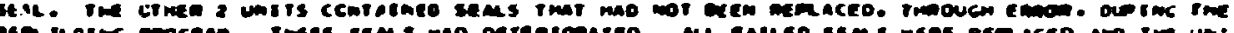
ervinge ro seinter.

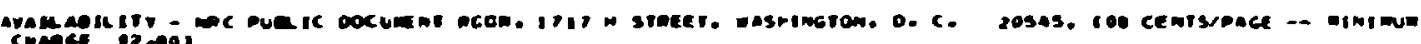

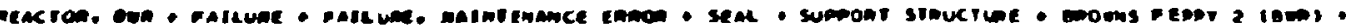

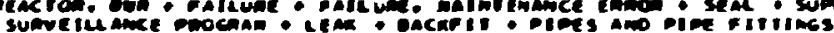

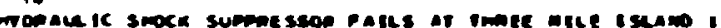

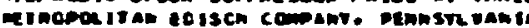

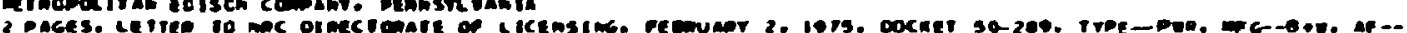
Geenei issoc.

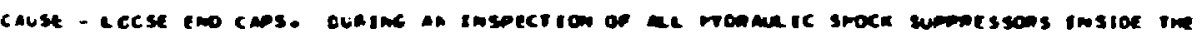

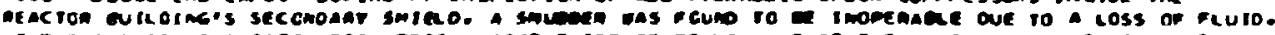

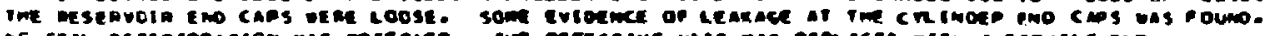

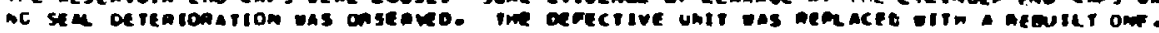

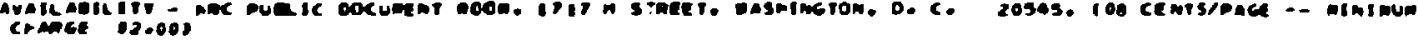

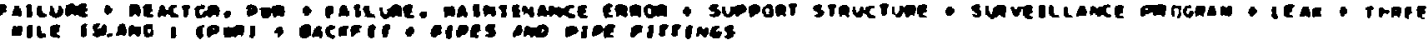




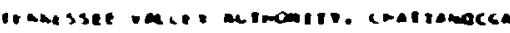

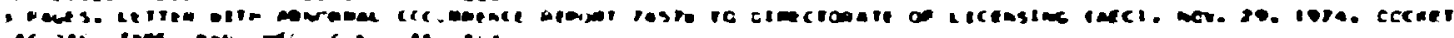

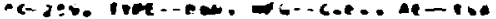

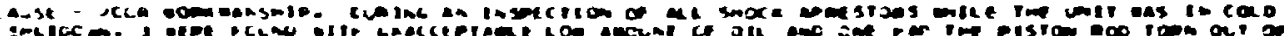

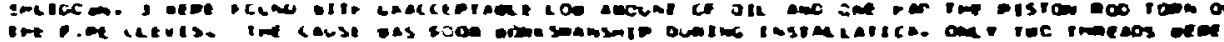

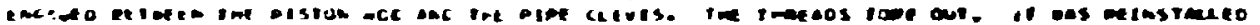

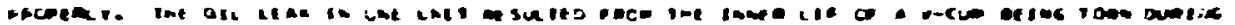

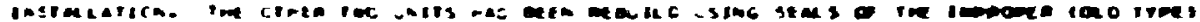

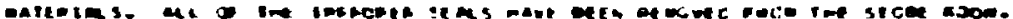

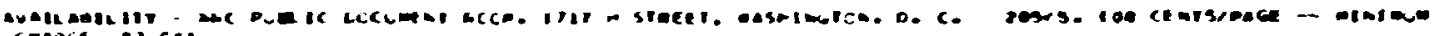

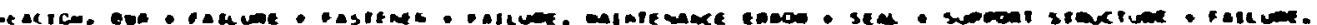

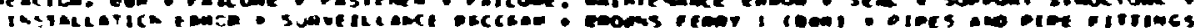

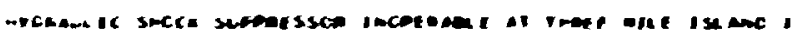

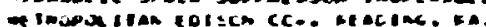

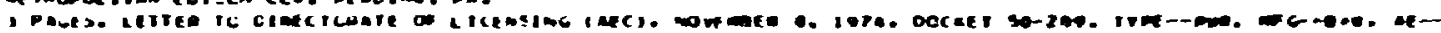
biketionsect

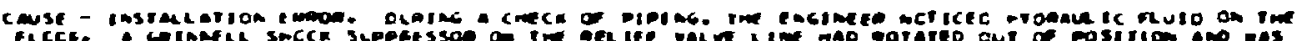

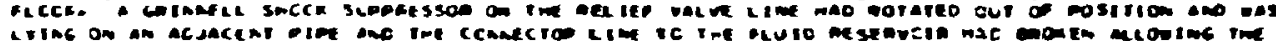

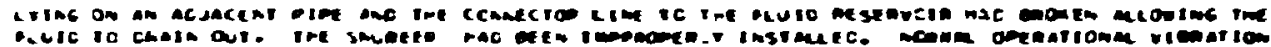

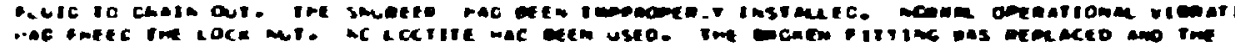

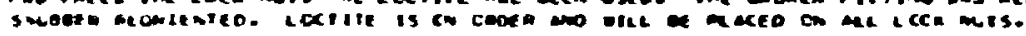

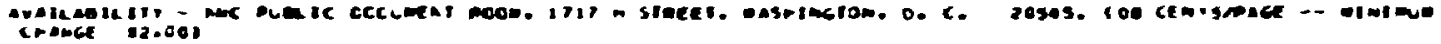

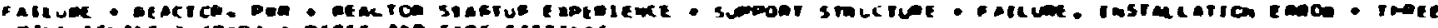

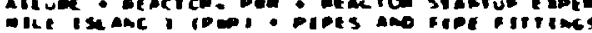

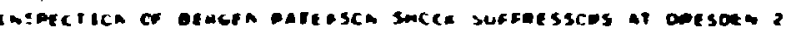

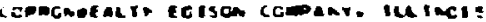

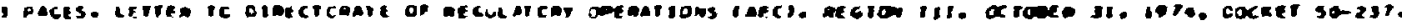

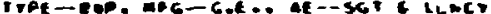

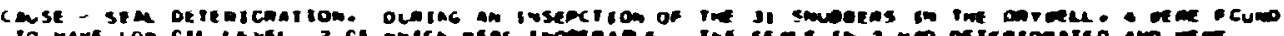

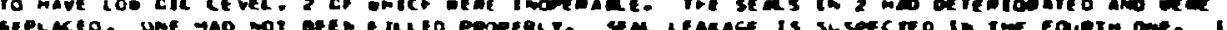

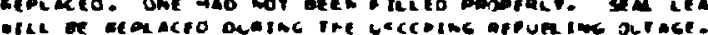

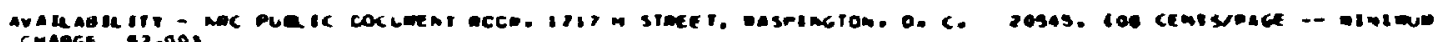

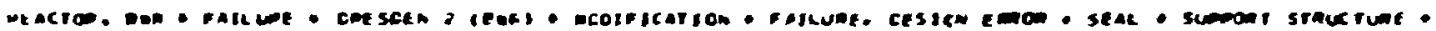

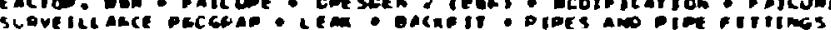

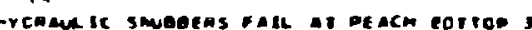

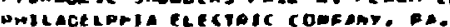

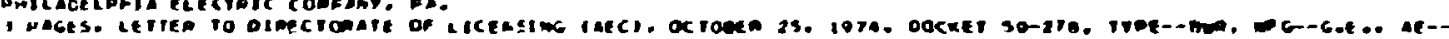
orcriel

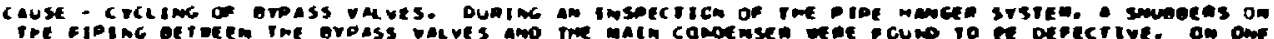

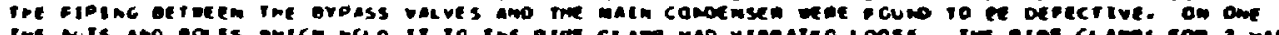

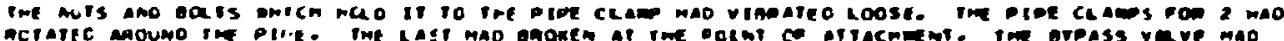

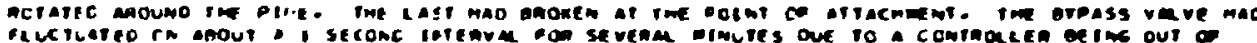

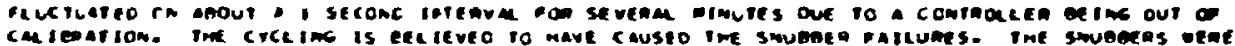

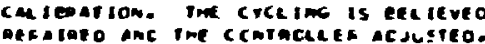

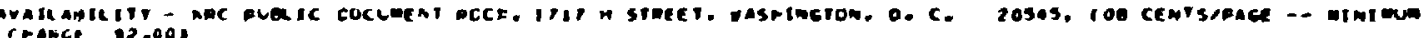

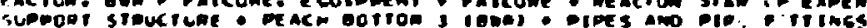

12

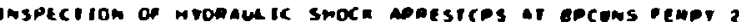

Tennisset vale, aurhcaitr, eratiamocse

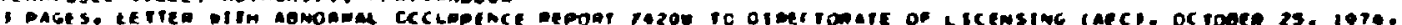

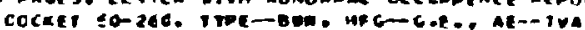

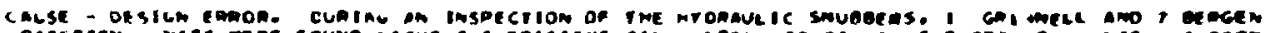

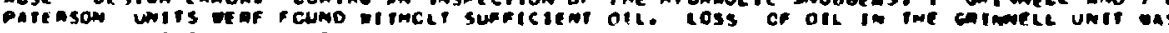

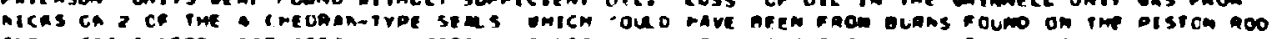

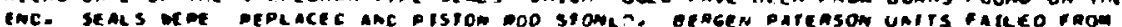

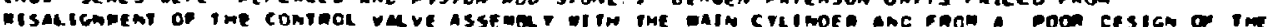

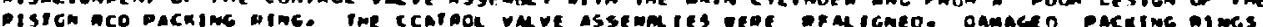
ofer aeblaceo. 


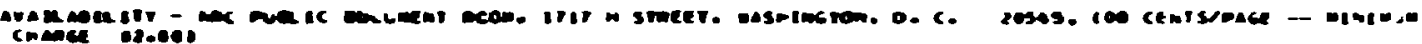

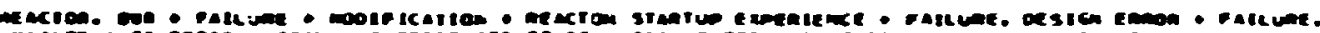

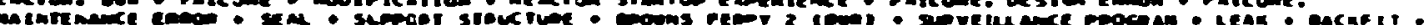
Dins ane eice eiriencs

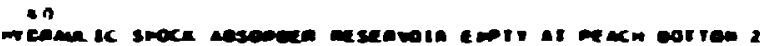

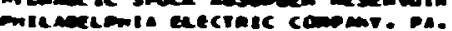

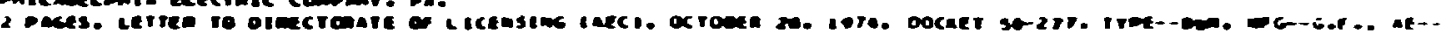
excmie

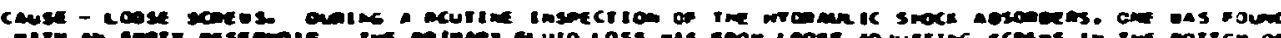

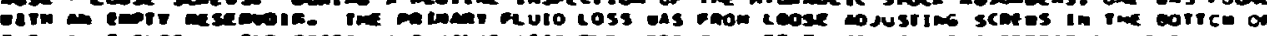

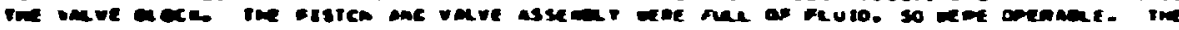

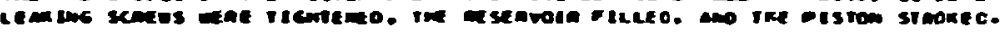

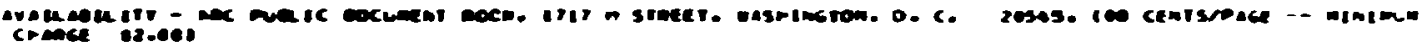

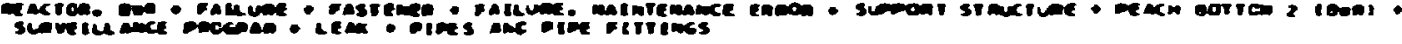

1

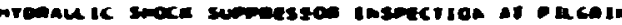

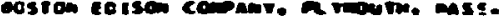

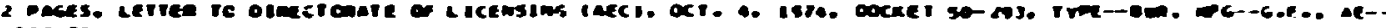
otcries

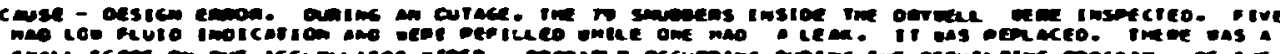

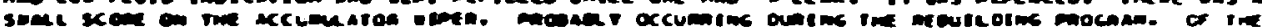

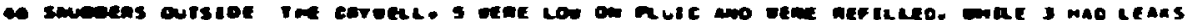

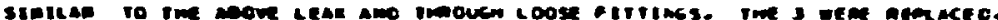

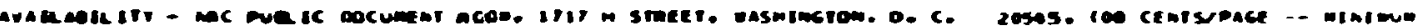
Cresce is os:

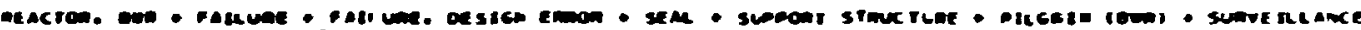

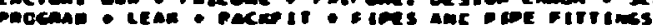

12

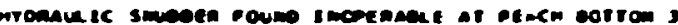

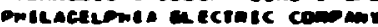

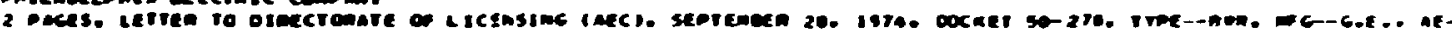
-oecririe

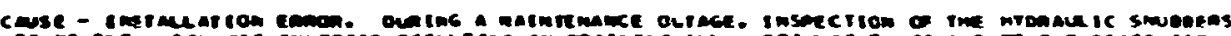

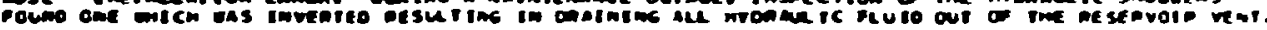

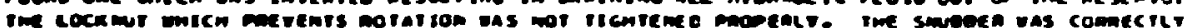

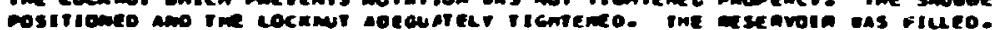

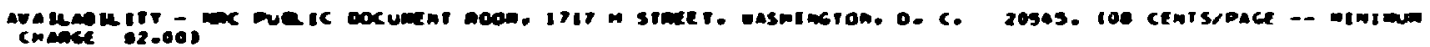

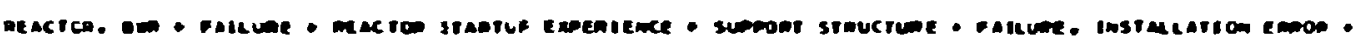

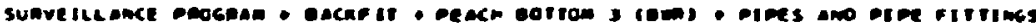

43

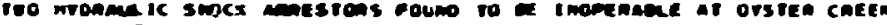

renser centam note 6 cichi comont

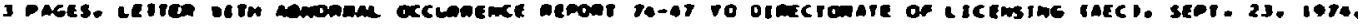

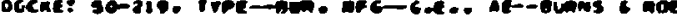

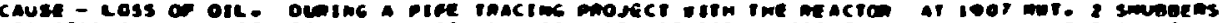

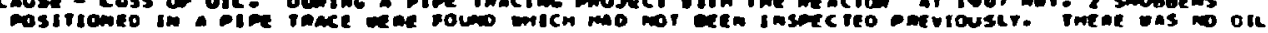

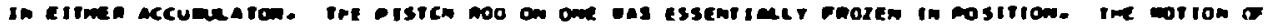

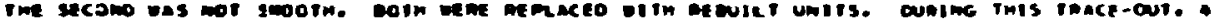

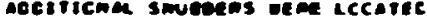

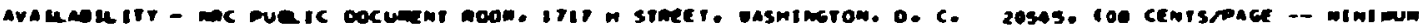
Crance es -eop

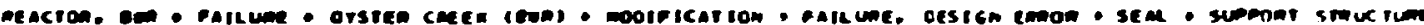

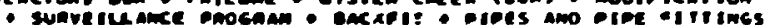

46

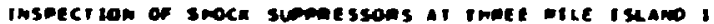

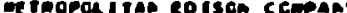

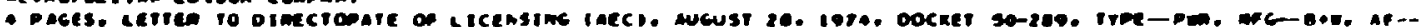
Giecer issoc.

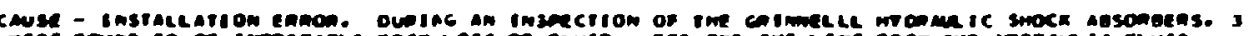
mere

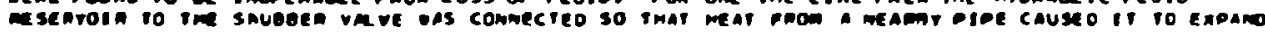

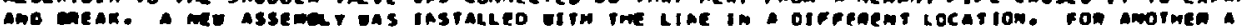




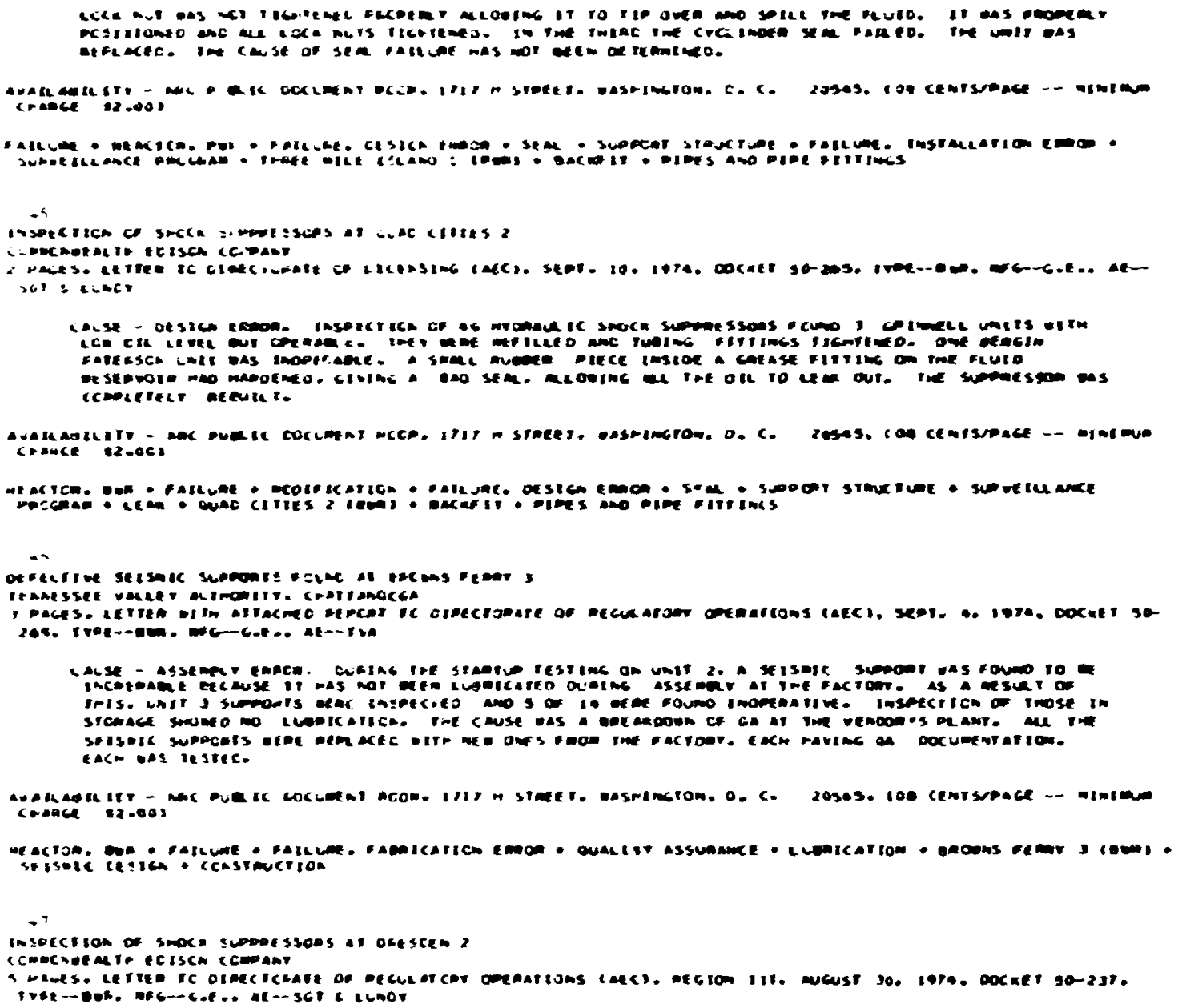

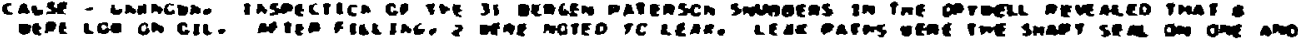

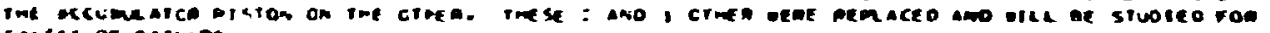
cacses of otithe

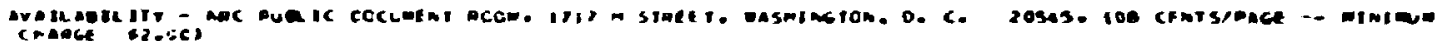

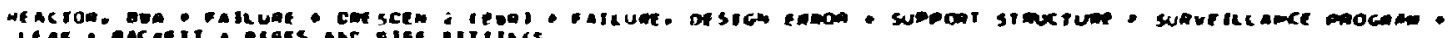
41

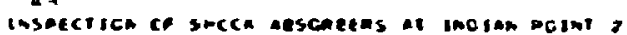

cinsoldoute oison comant

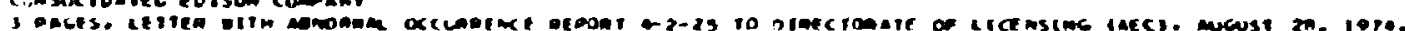

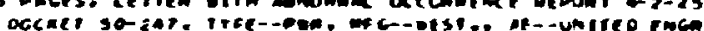

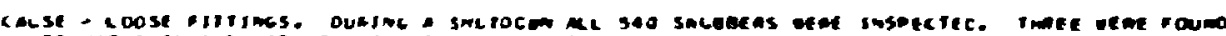

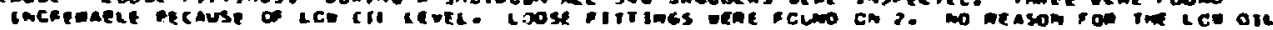

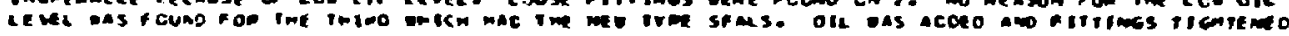
ca ne ramet.

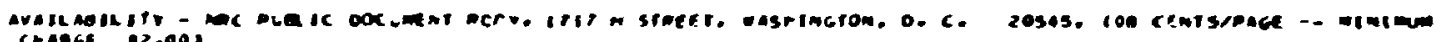

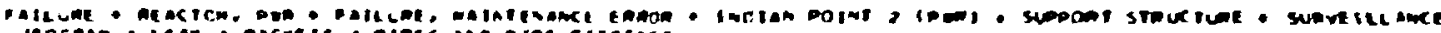

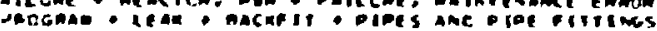

$+2$

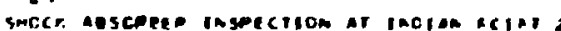

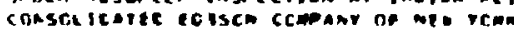

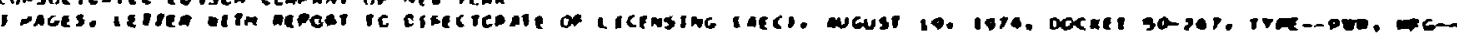
ust.. ae-cuastic enea 


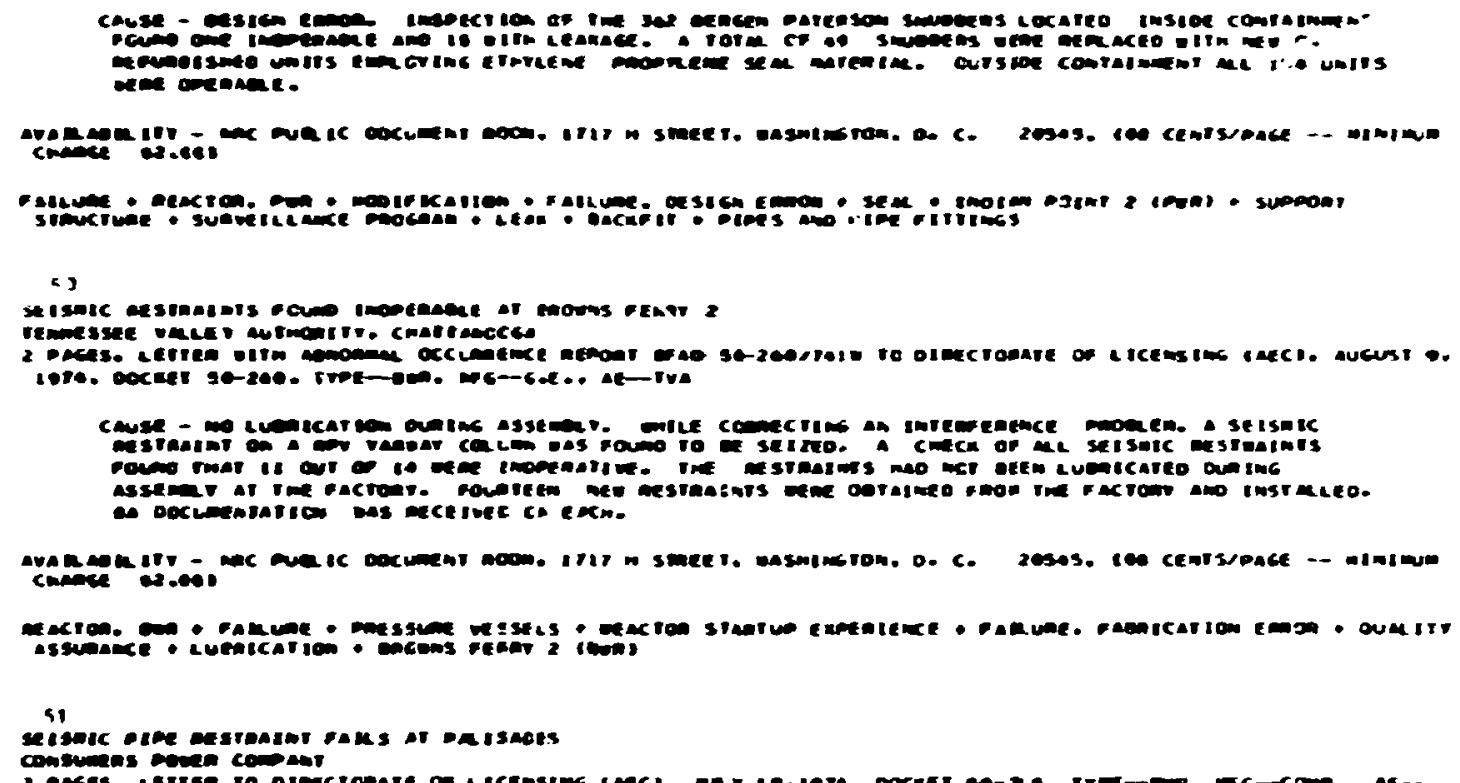

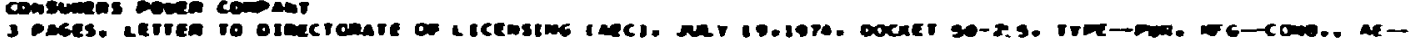
ecerree

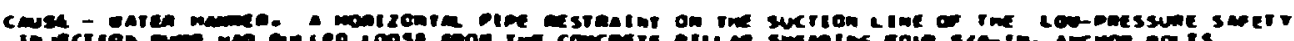

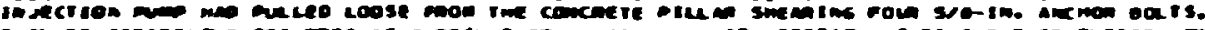

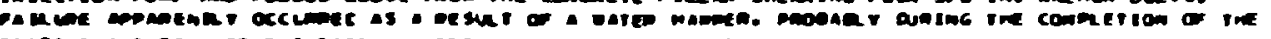

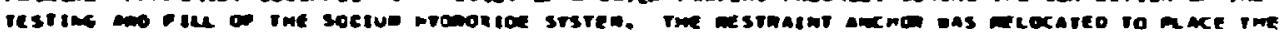

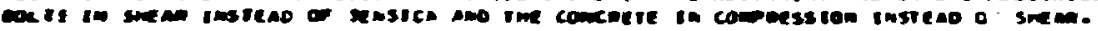

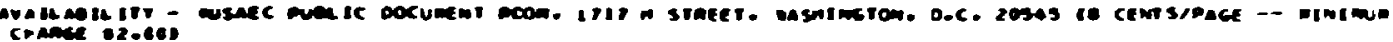

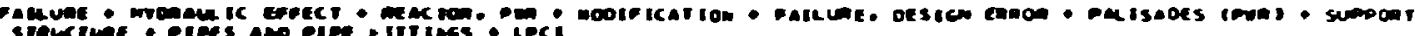

52

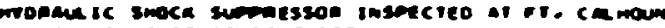

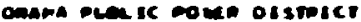

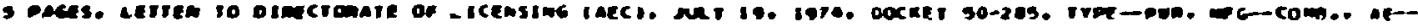
GI00s. mike

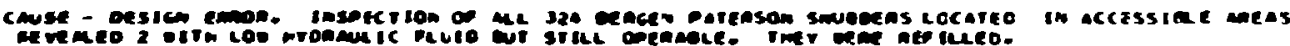

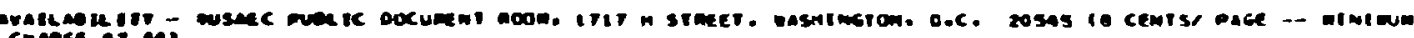

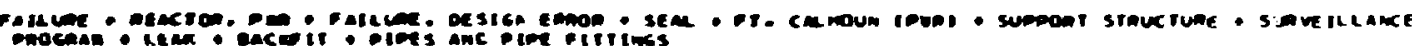

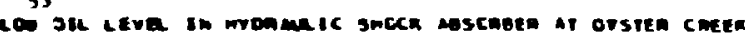

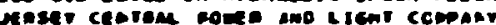

3 aces.

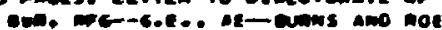

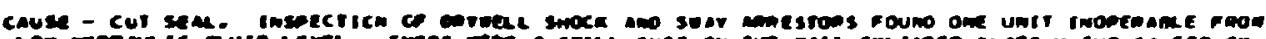

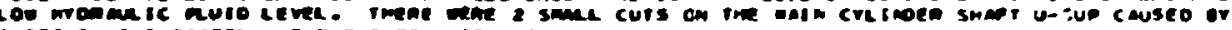

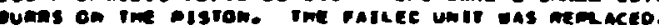

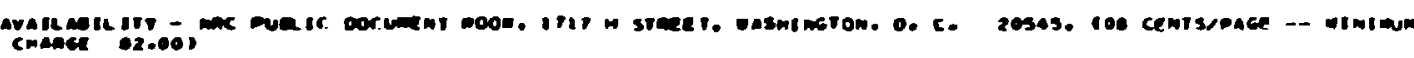

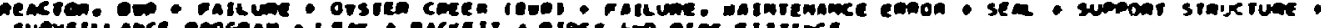

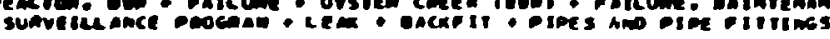

54

Obe Lear in wronmelc smock suronessen or aAmoco

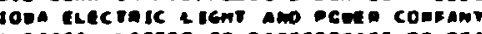

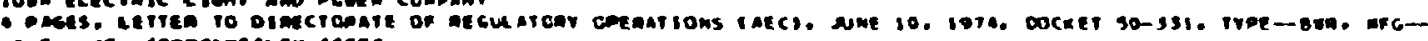

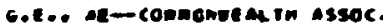

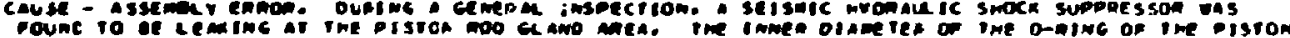

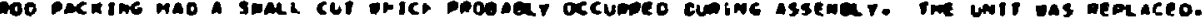




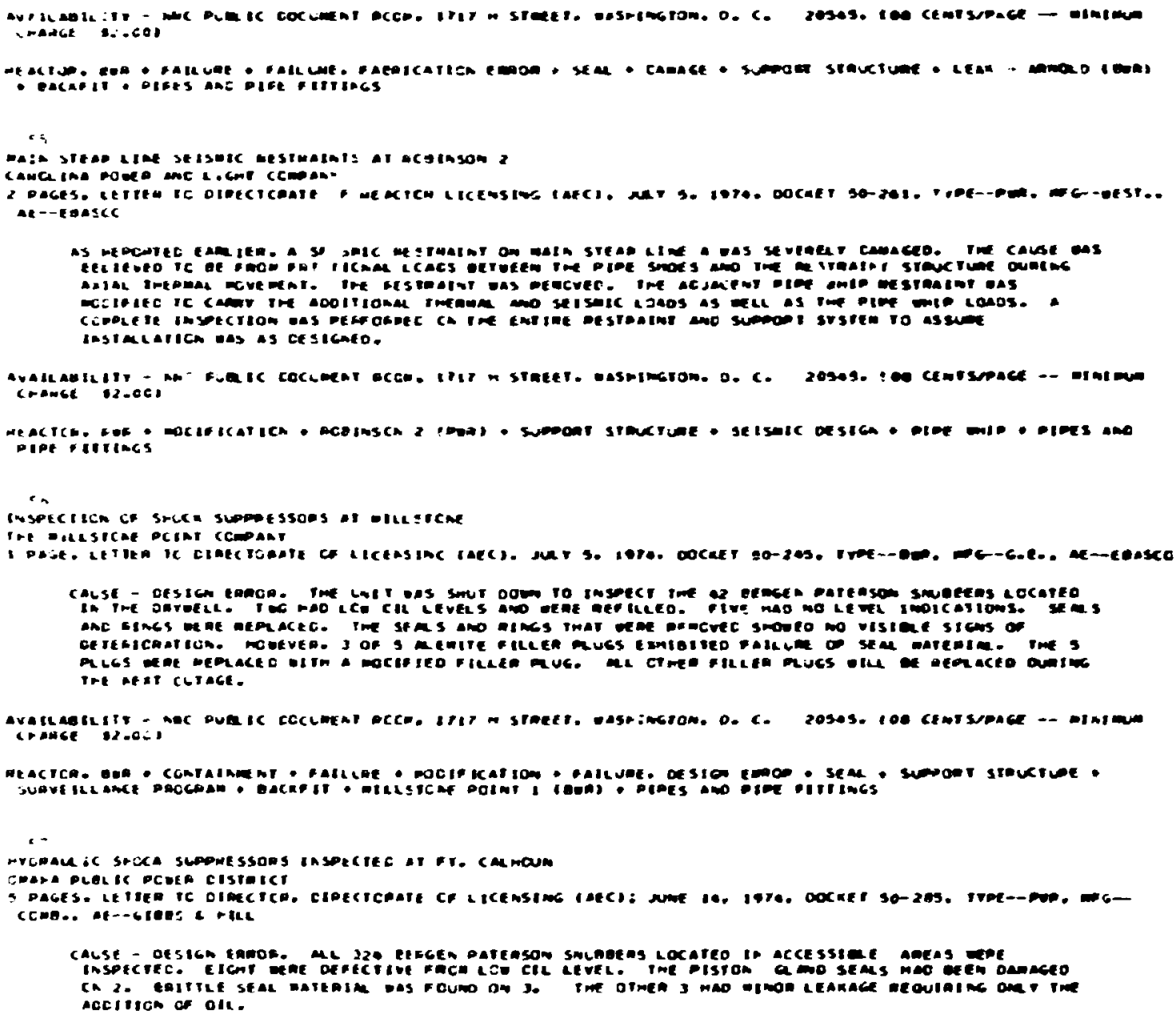




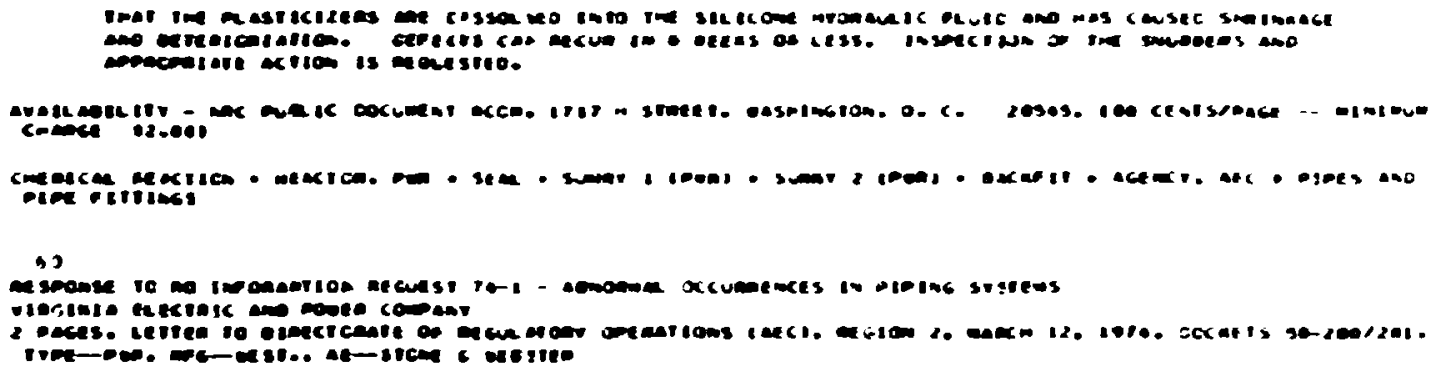

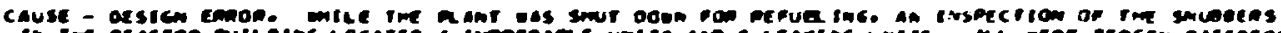

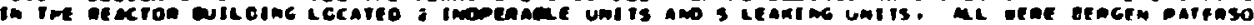

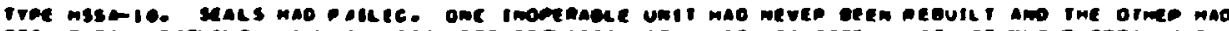

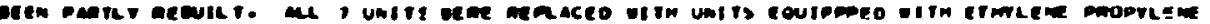
sens.

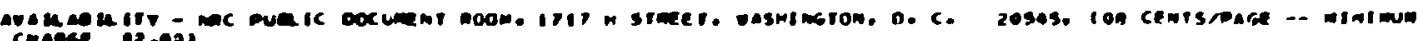

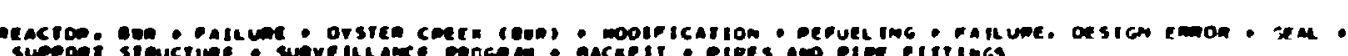
he

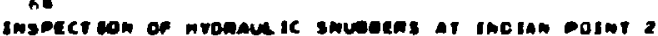

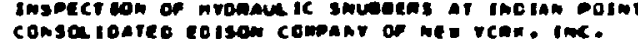

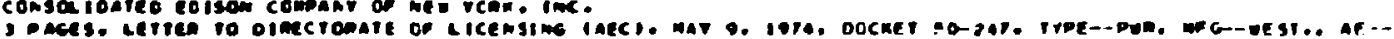

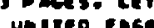




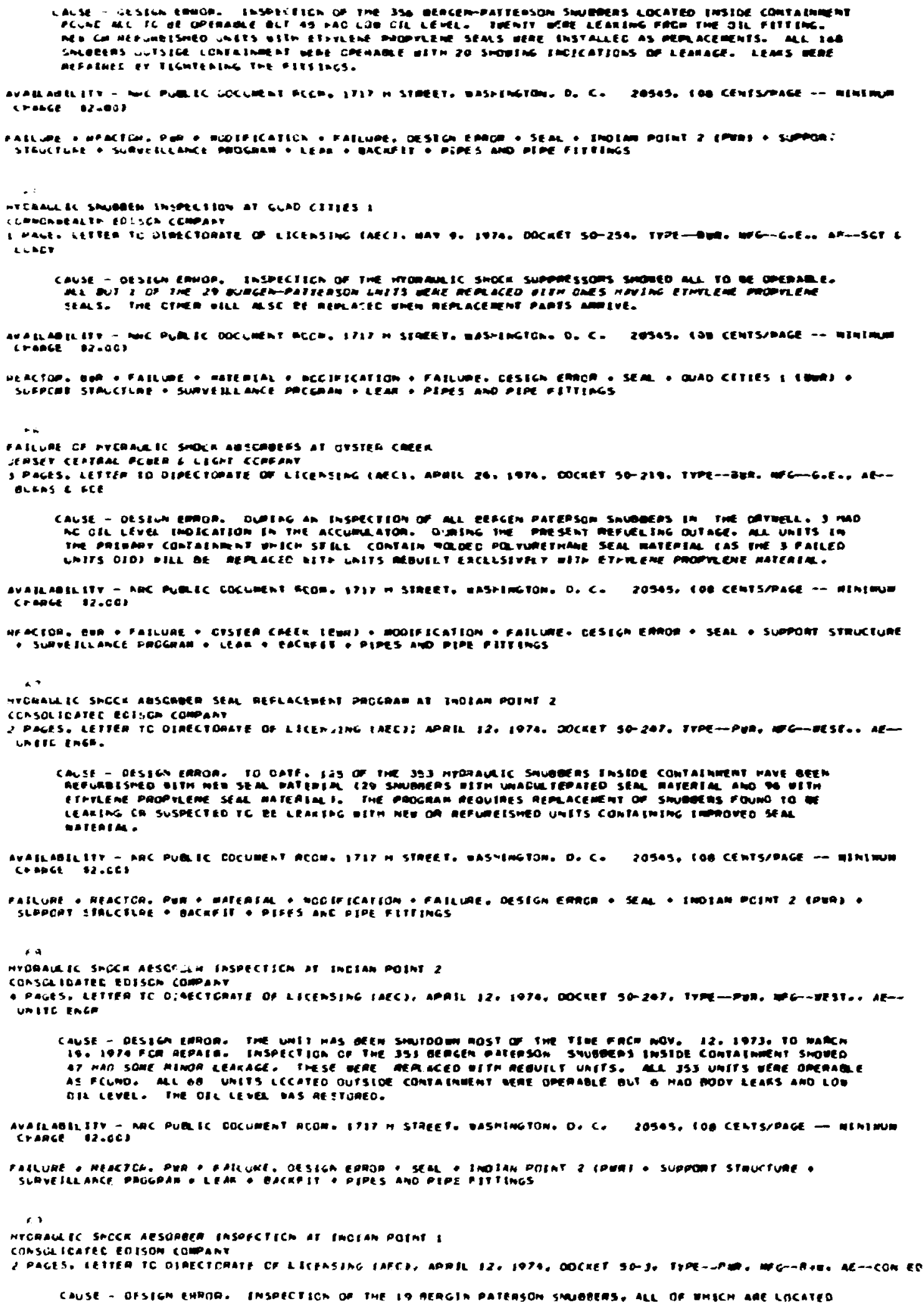




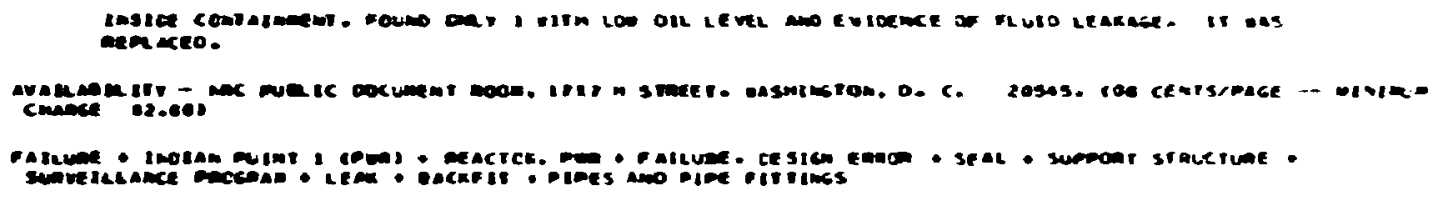

20

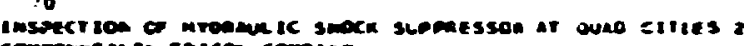

conomeen re eos sen coman.

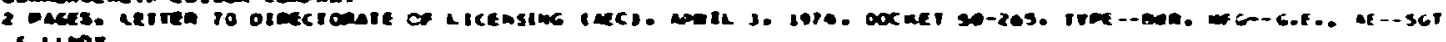

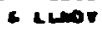

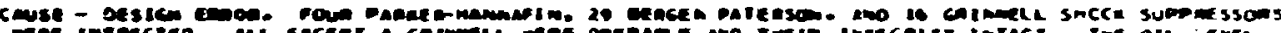

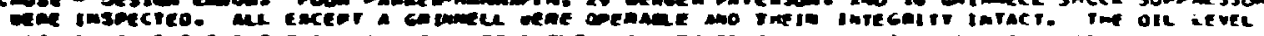

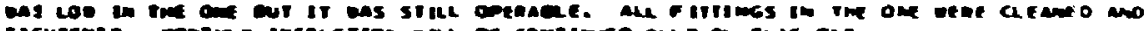

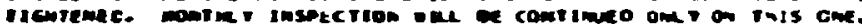

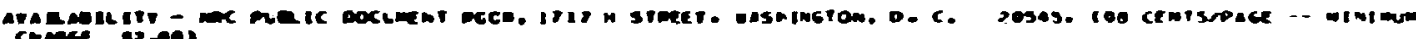
12001

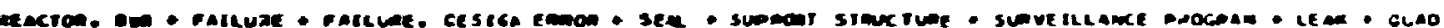

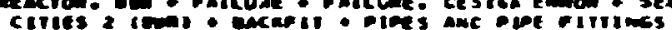

7

eceullorins or shock sumessons at pilckin

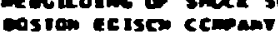

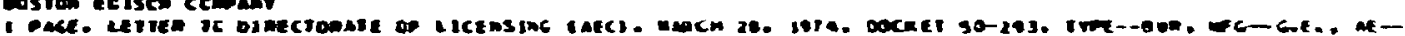
eeceres

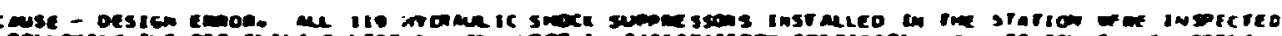

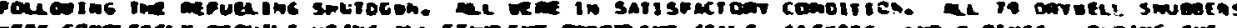

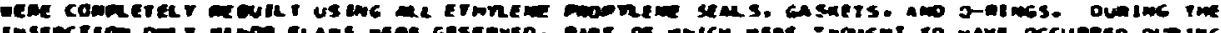
cessas

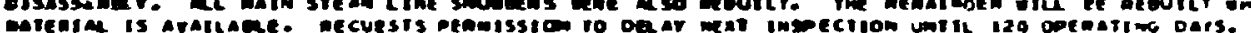

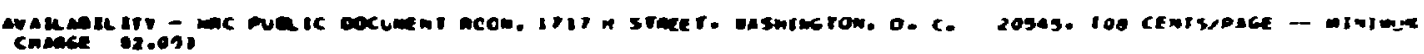

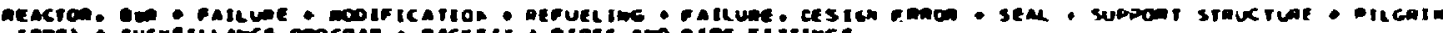

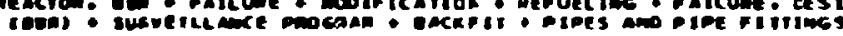

12

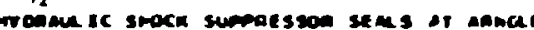

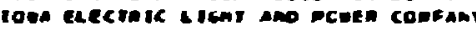

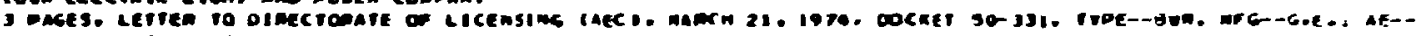
cconenge alim ossoc.

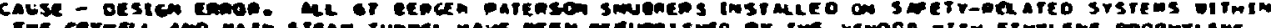

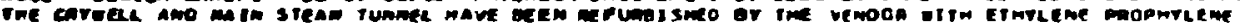

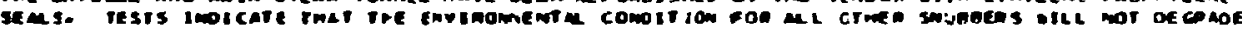
The instrale sems.

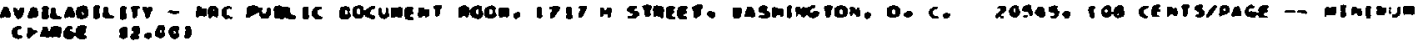

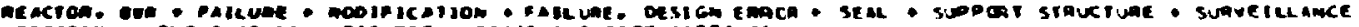

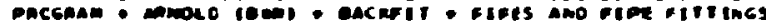

7

Inspection of mromaulec smock supenesscas ar inesoen 3

combonveral it colson compant

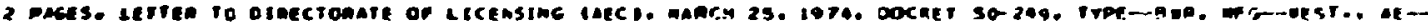
sci s Lmor

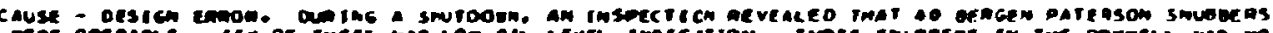

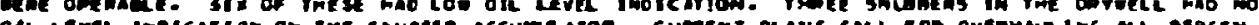

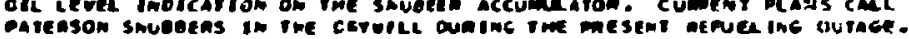

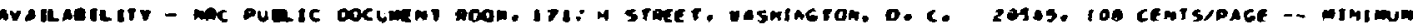
Crance 12.001

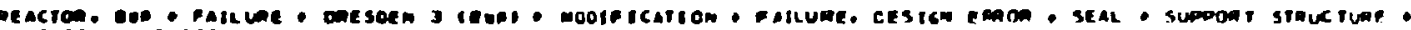

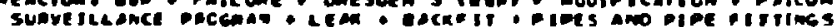

7.

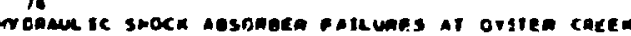

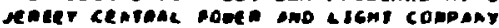

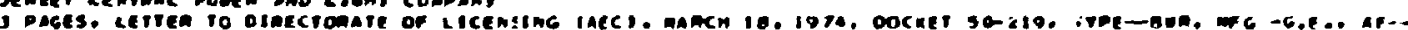
auns ane ece

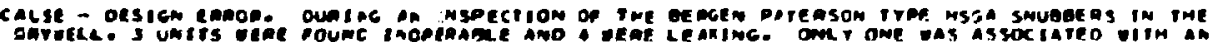




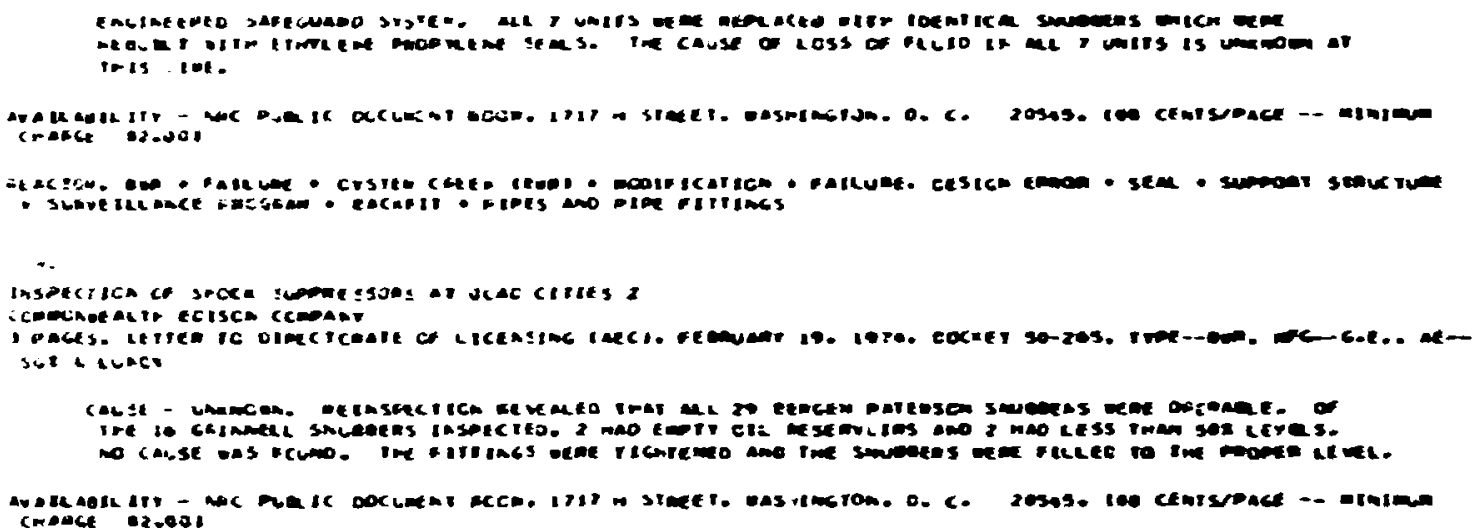




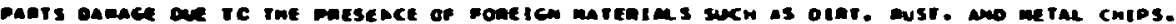

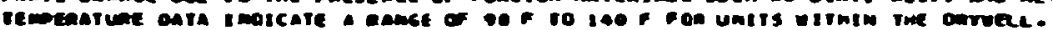

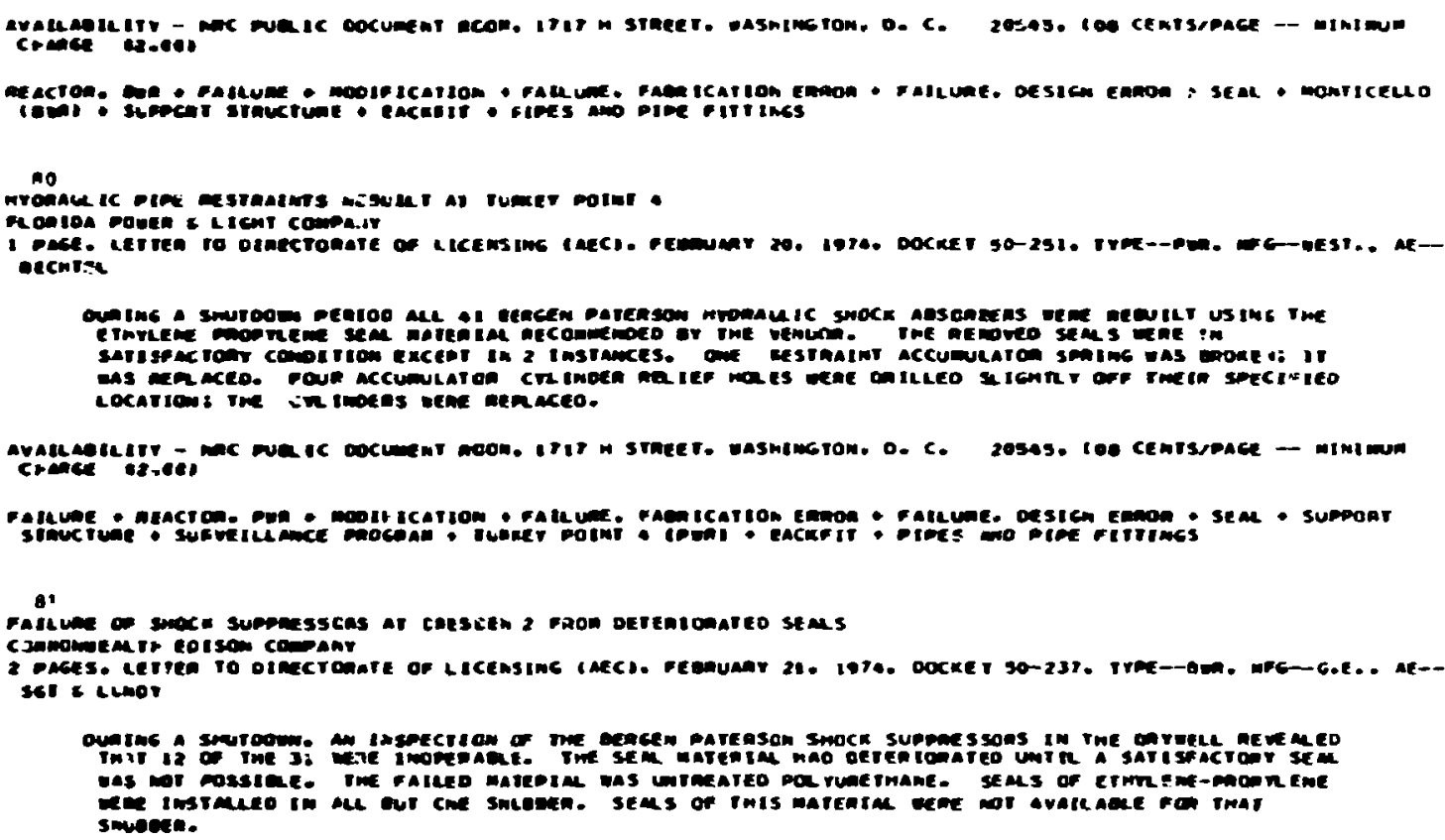

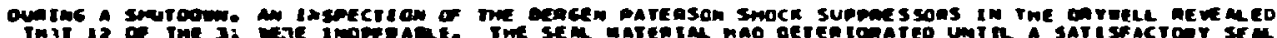

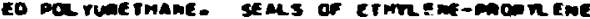

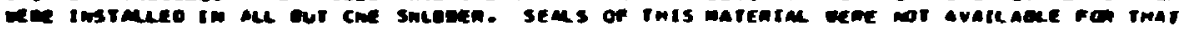
smuecte.

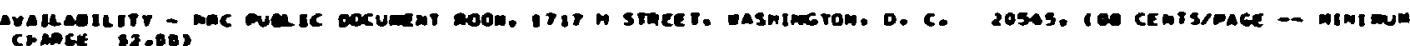

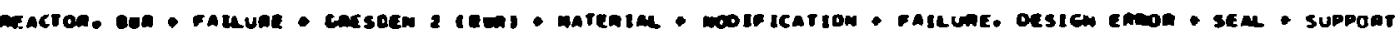

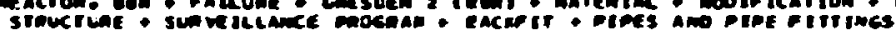

A2

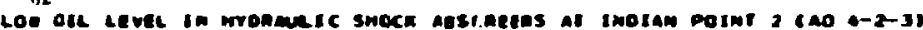

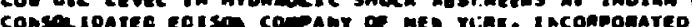

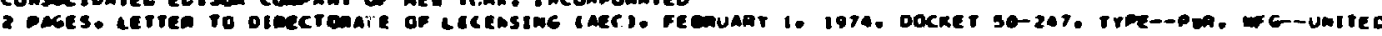
encrester

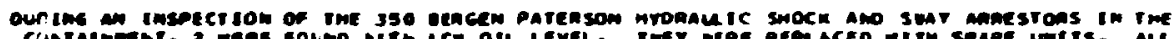

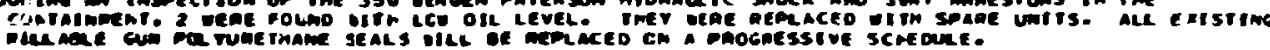

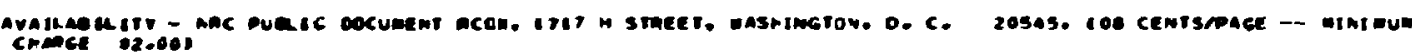

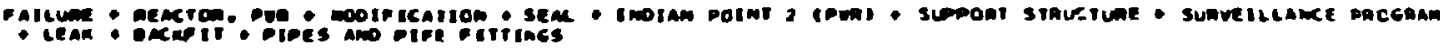

A)

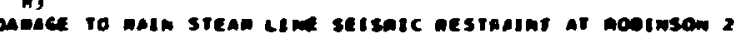

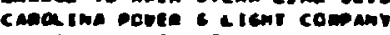

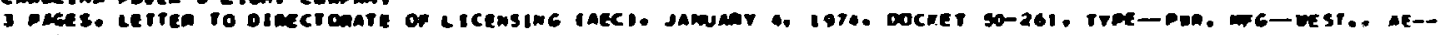
toasco

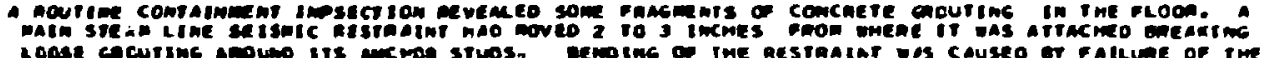

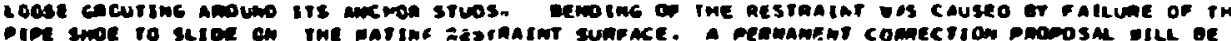

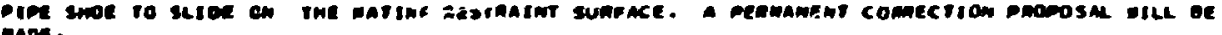

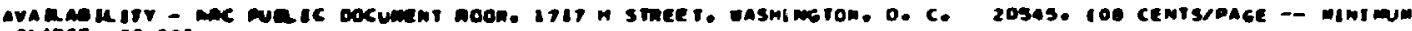
crance 20 ast

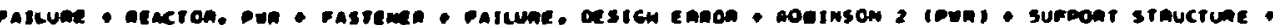

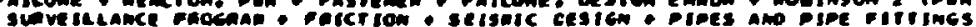

an

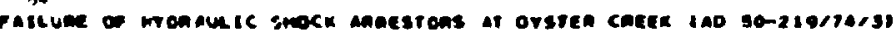

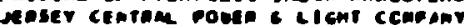

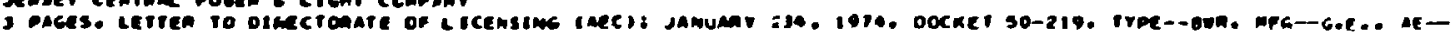
ucuns

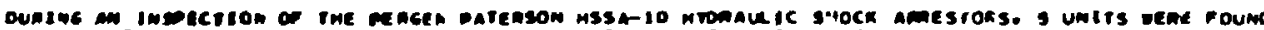

IIn mo jucocon 


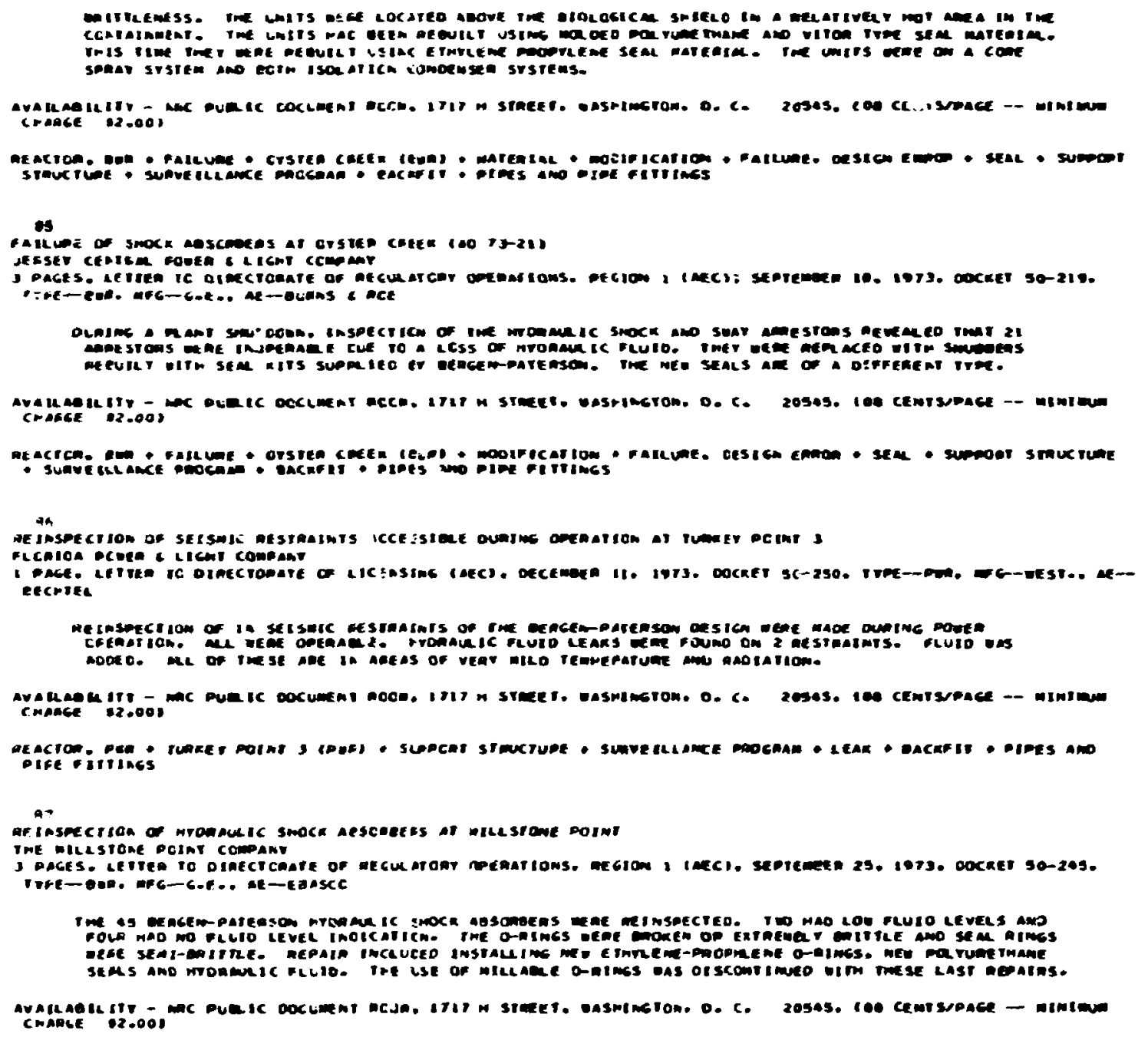

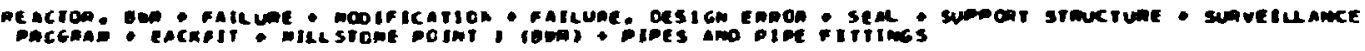

Ba

WE Imspection of smock suppenessons ir Fi. CMrmoun

omama alelic moge orstaici

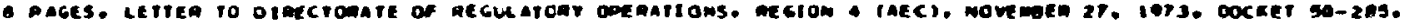

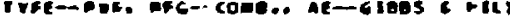

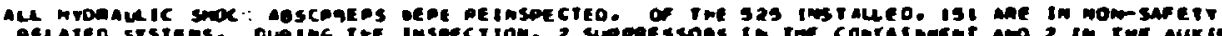

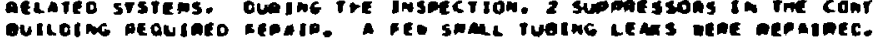

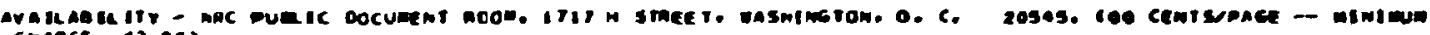

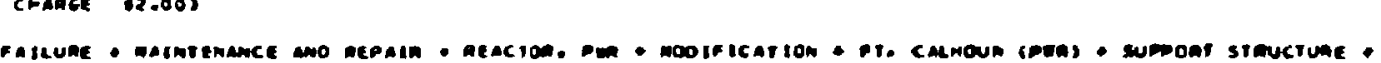

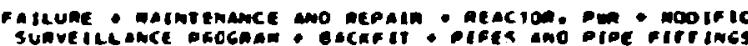

AT

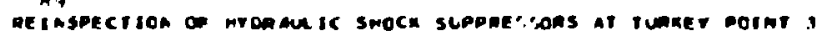

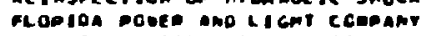

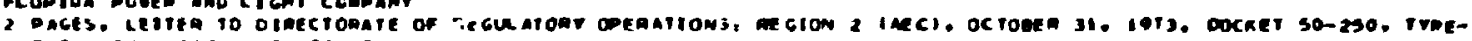

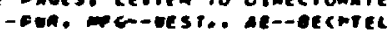

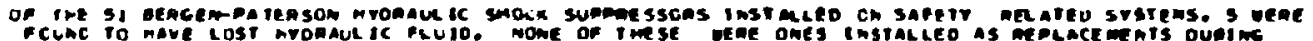

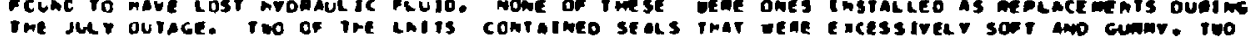

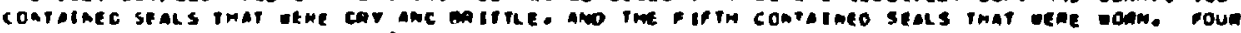

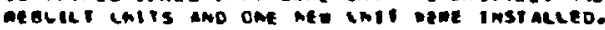




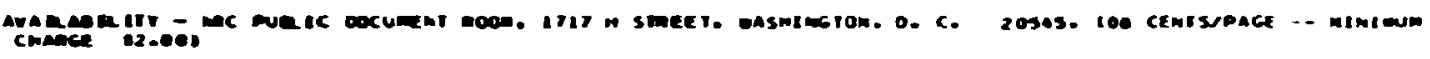

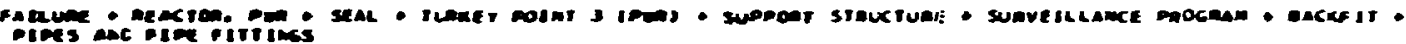

80

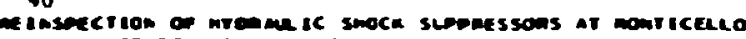

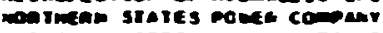

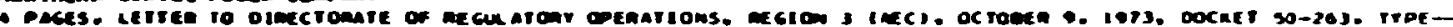

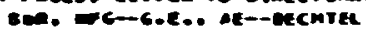

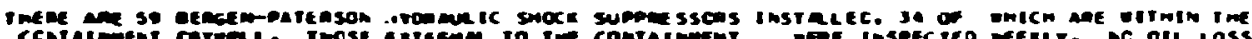

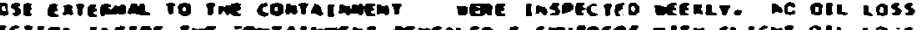
was

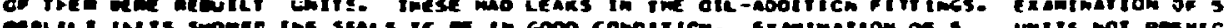

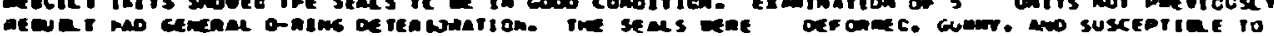

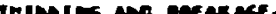

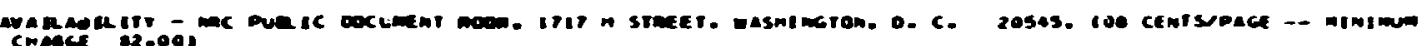

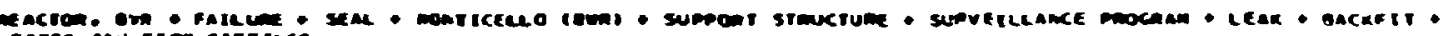

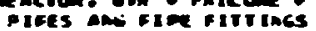

91

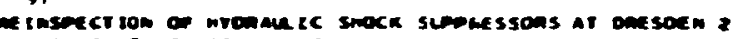

componne Mit ediscr ccomant

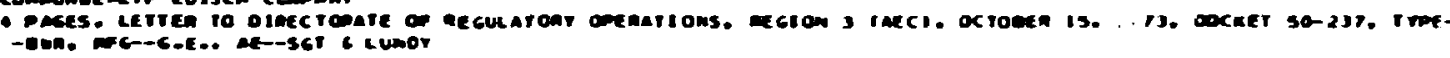

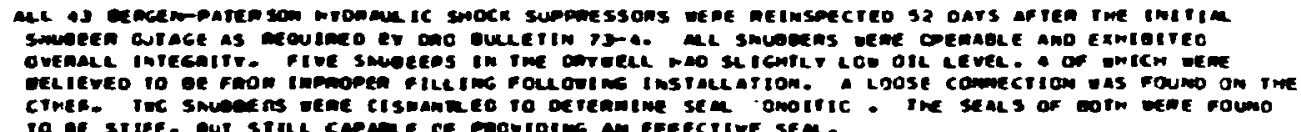
the

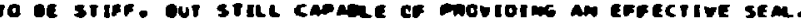

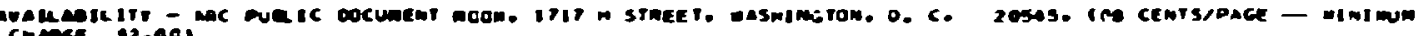
(2)ecs

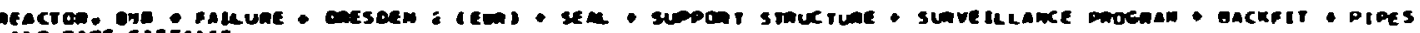
no pipe etritings

92

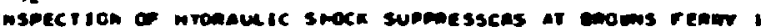

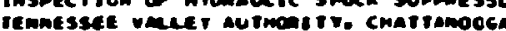

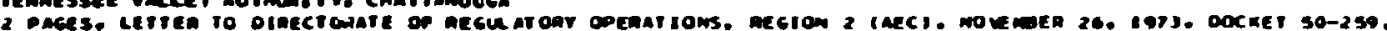

res-

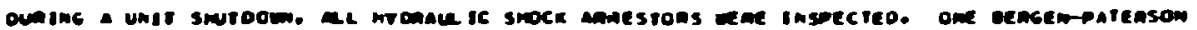

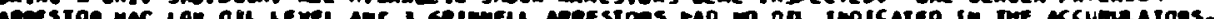

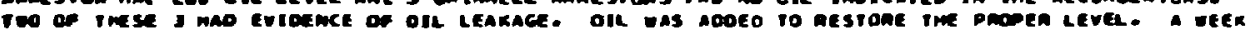

raren

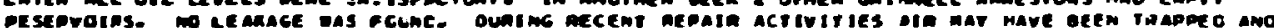
is ace escapint.

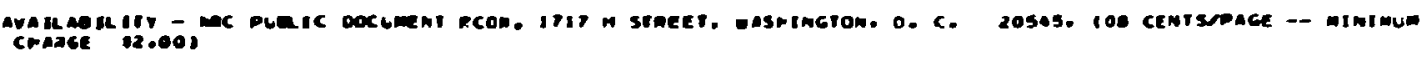

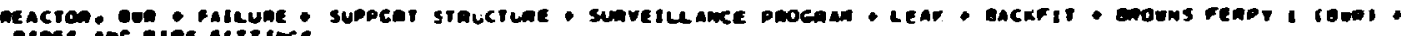
Dives anc oipe pistims

93

mowace ec shoce Absoacen inspectich at eileain

vos rep eciscm ccopant

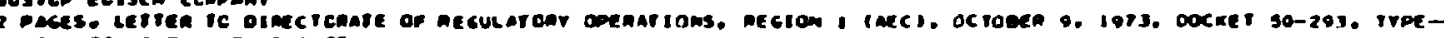

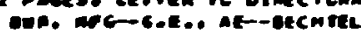

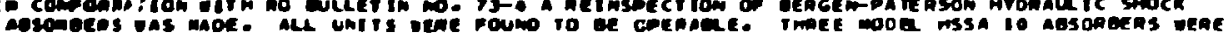

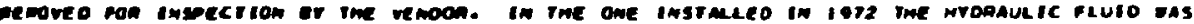

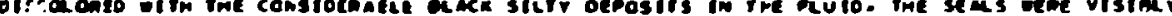

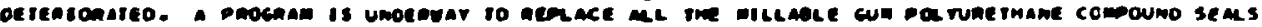

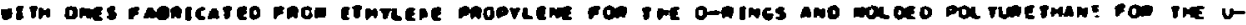
cues ans uspens.

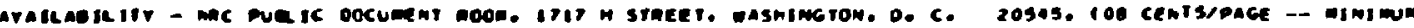
comete

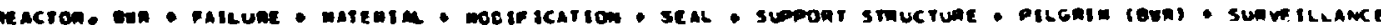

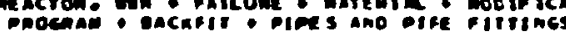

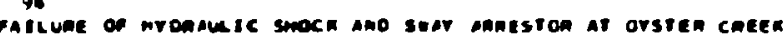

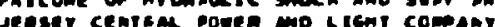




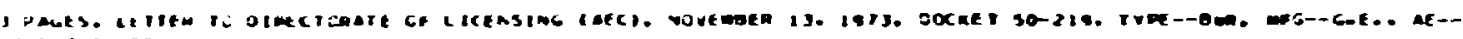
elans 4 cer

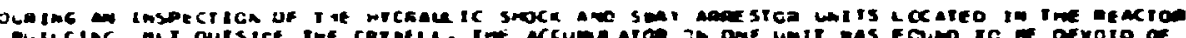

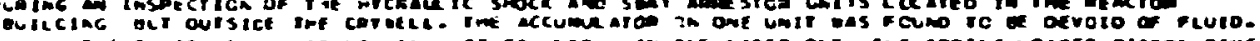

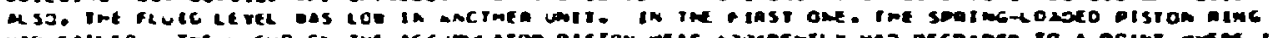

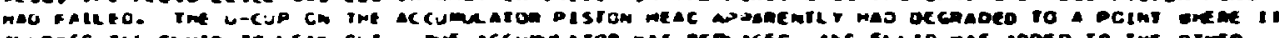

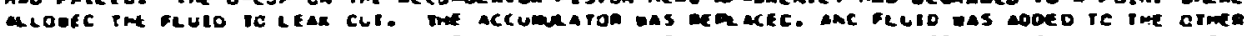

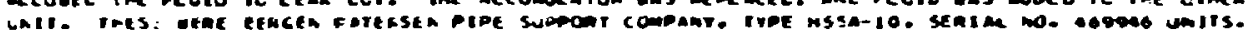

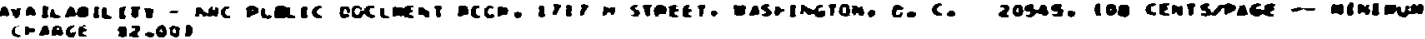

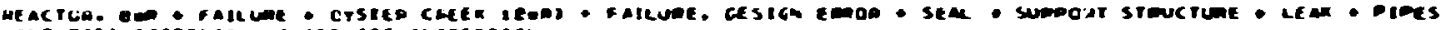

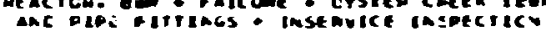

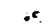

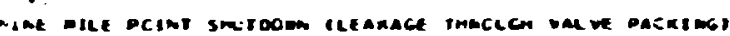

hiegane ecraper foceka compceatica

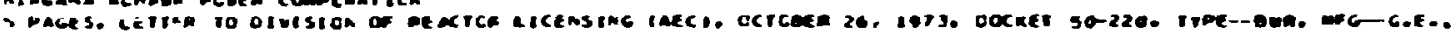

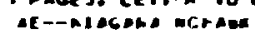

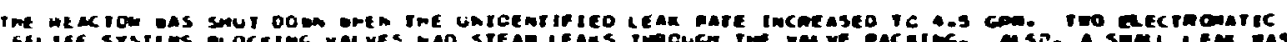

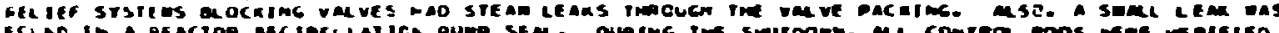
Fend sn s re

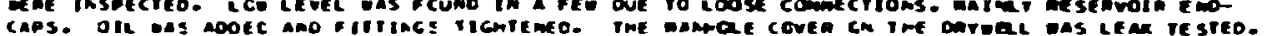

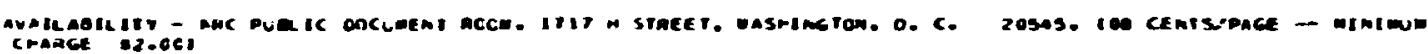

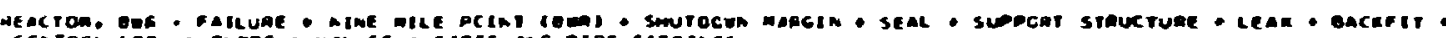

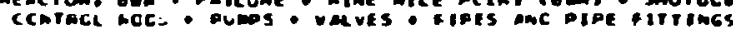

it

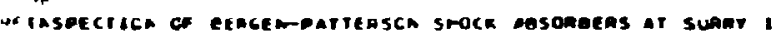

viacimis tectrec ano pone cCmpary

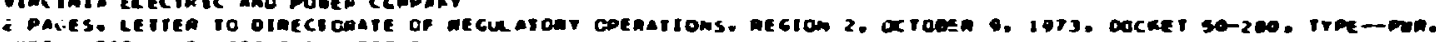
a c-oesi.. ae-sicae c oegsien

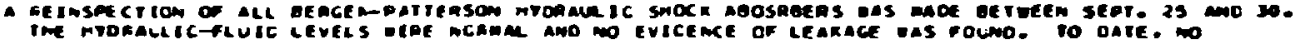

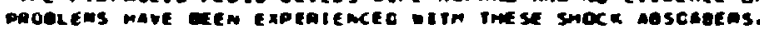

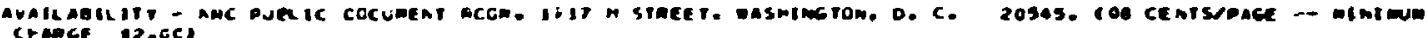

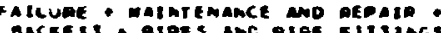

$\$$

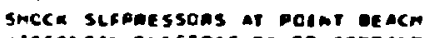

- isconsin elecreic poef ccopant

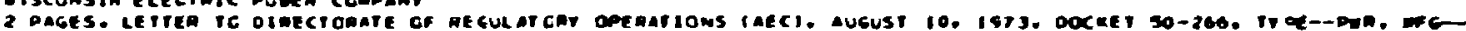
iesto. it-GeCries

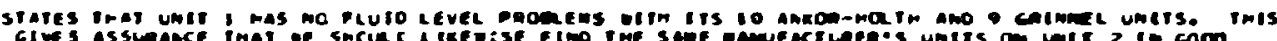
Gives assumane inal

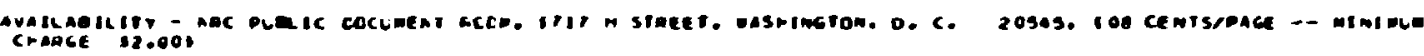

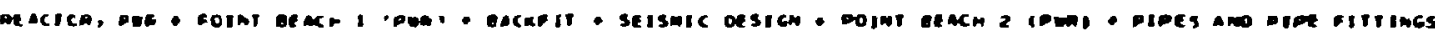

$n s$

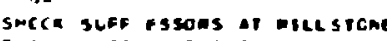

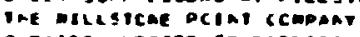

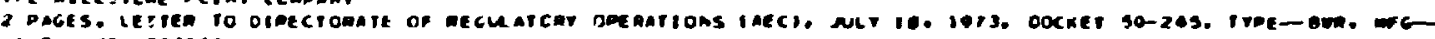
G.E... AE-Eessec

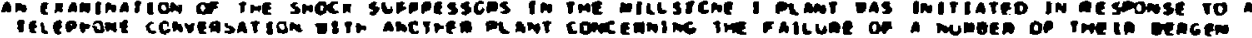

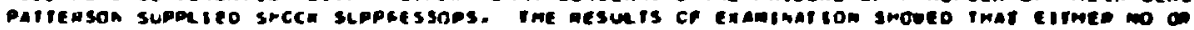

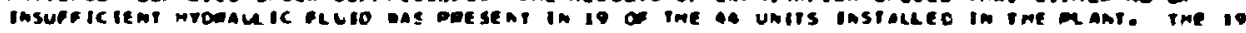

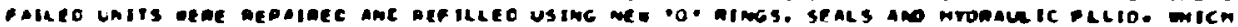

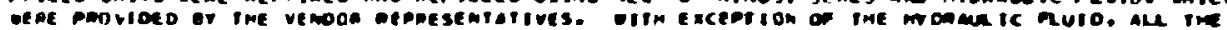

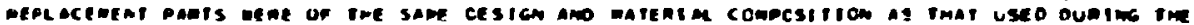

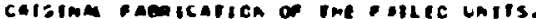

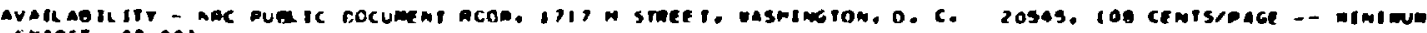
MAAcE 12.001

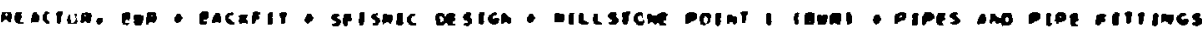


93

Face ir smora sumessons at menticelec

noetrene steres ncaen compant

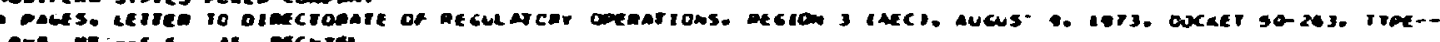

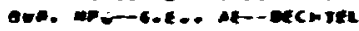

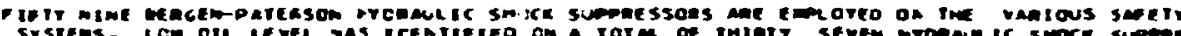

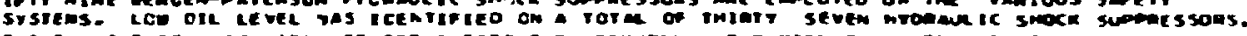

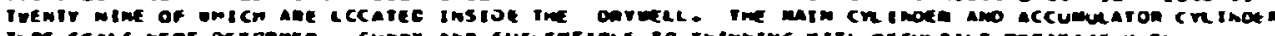

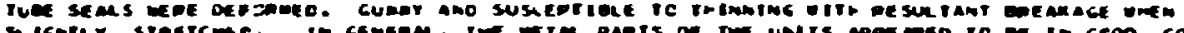

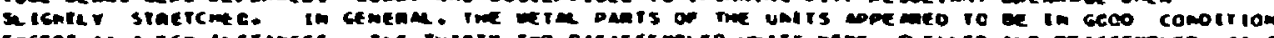

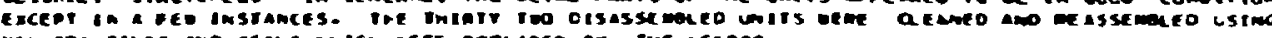

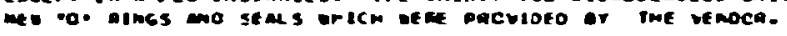

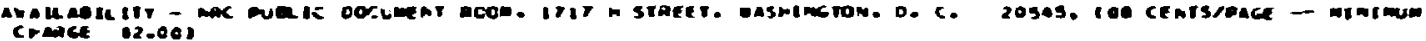

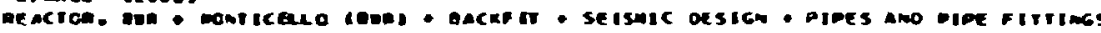

120

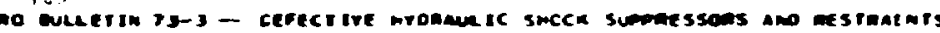

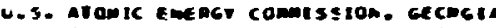

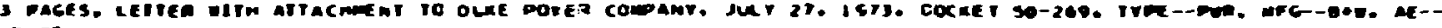
DLate

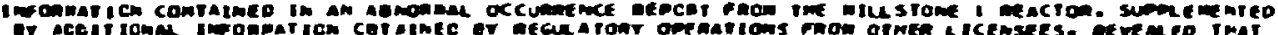

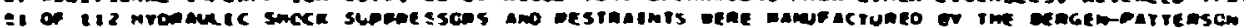

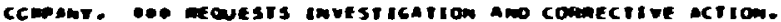
Avallesicist -

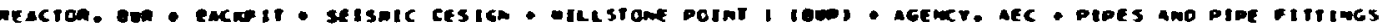

$\ln$

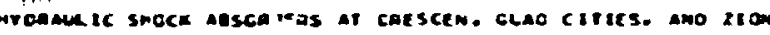

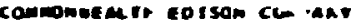

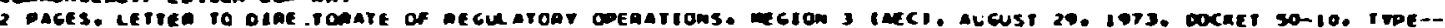

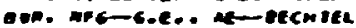

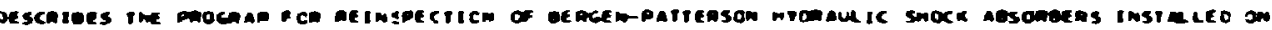
segati-hecatec ststeos.

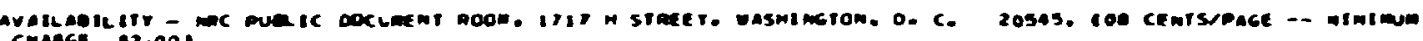

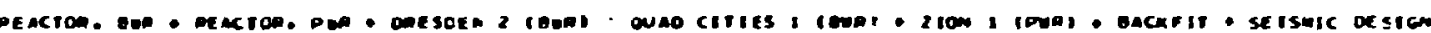

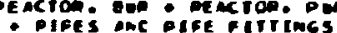

19

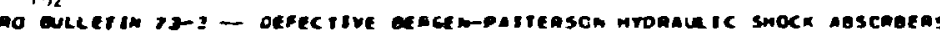

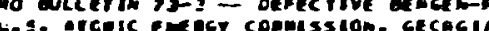

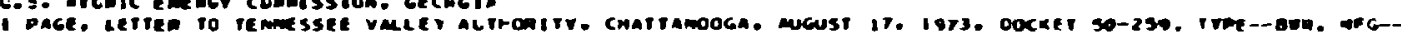
c.t. 0.100

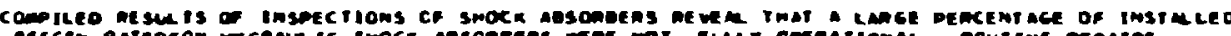

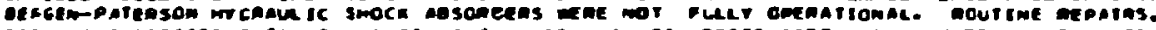

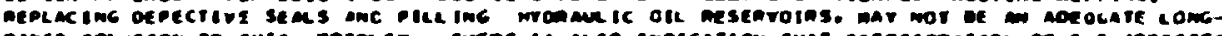

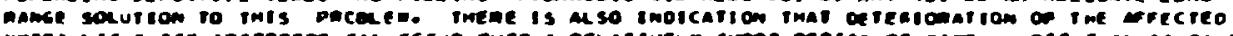

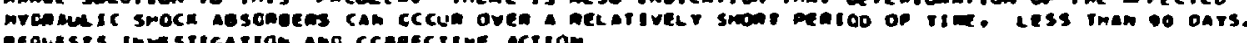

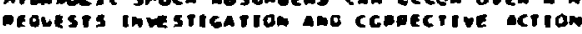

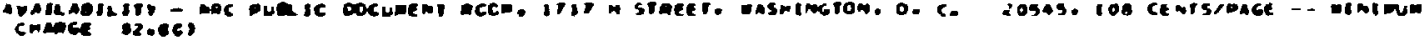

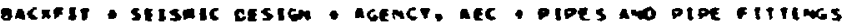

103

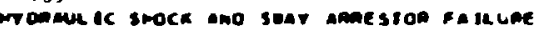

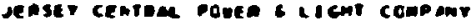

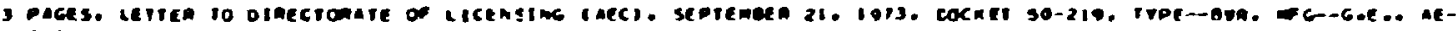
- evans soce

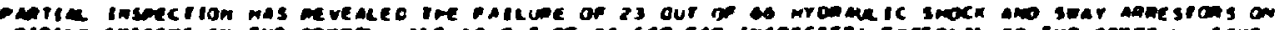

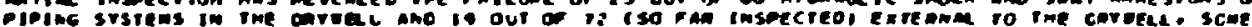

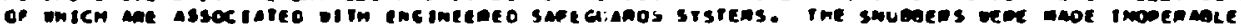

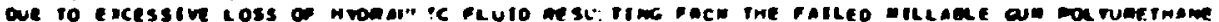

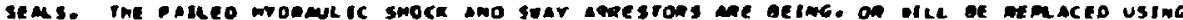

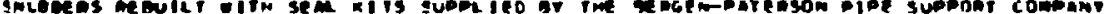

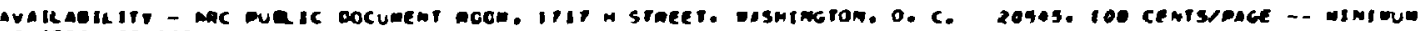

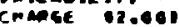

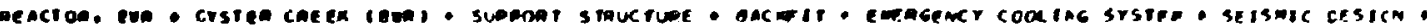
Dipes ino pire pirings 


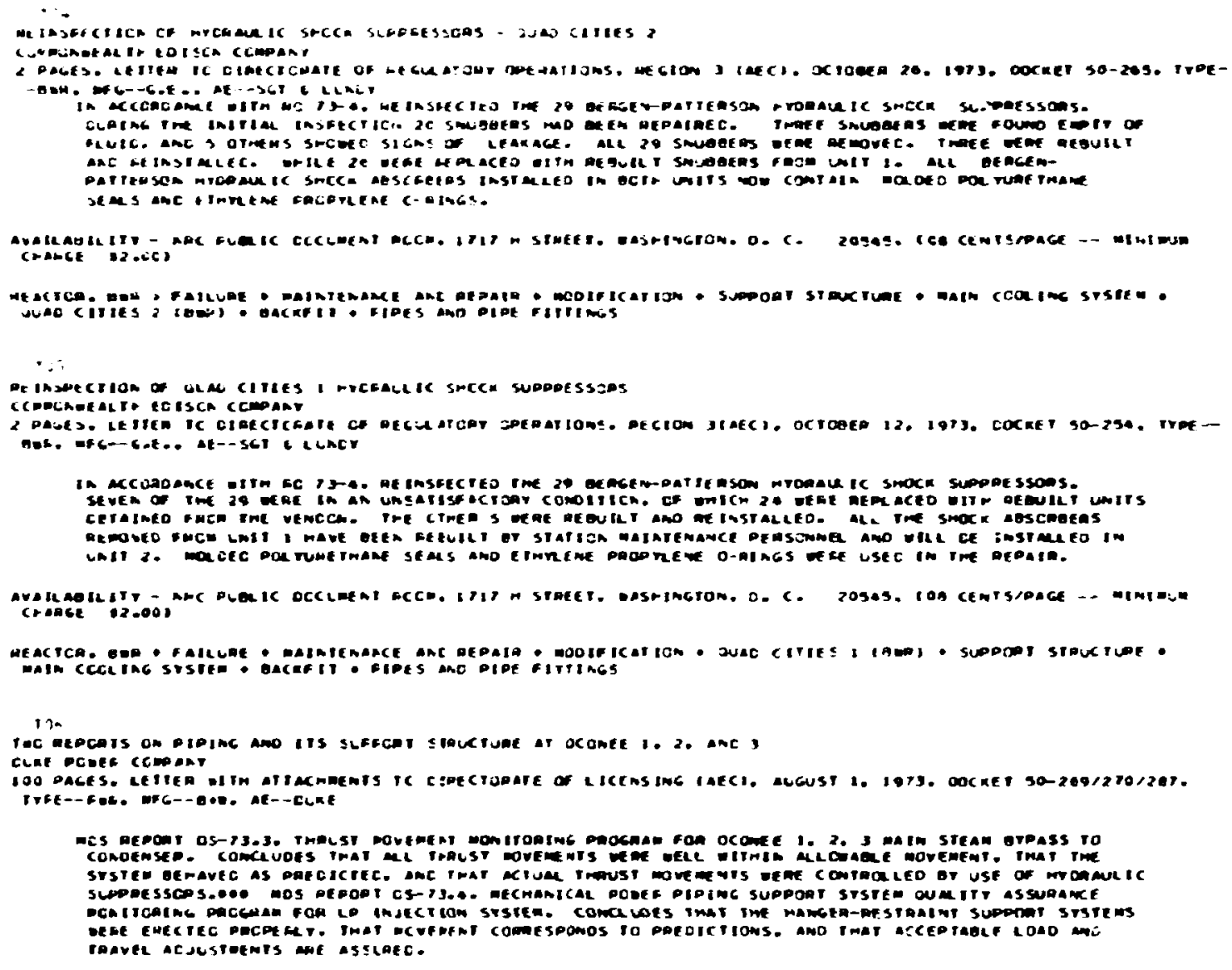

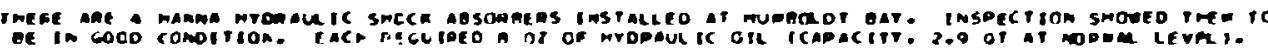

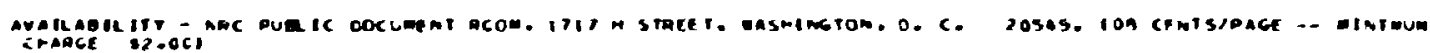

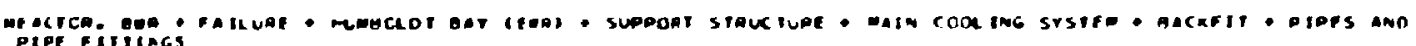

$1: j+$

malluee to Delo ice srock suppaesson at coesoen 3

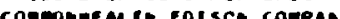

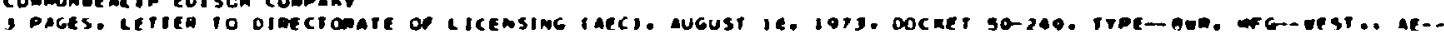
361 inAOT

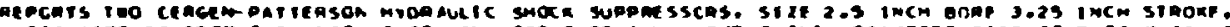

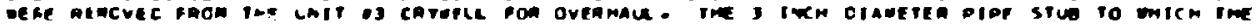

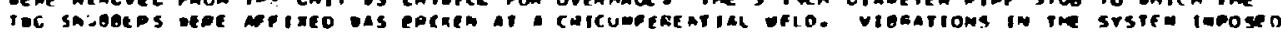

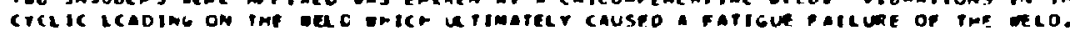

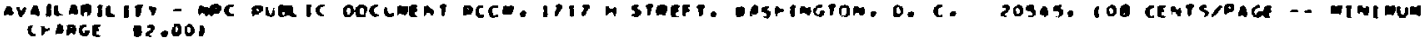

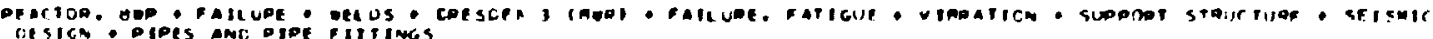


10.

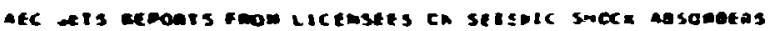

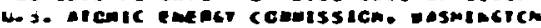

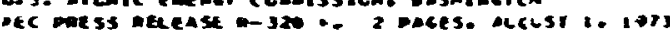

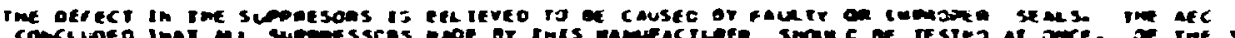

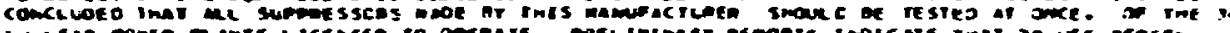

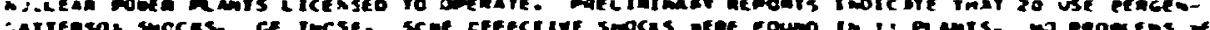

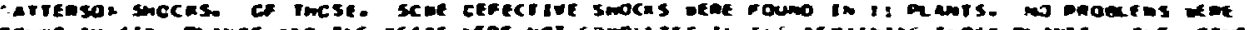

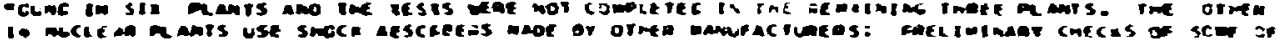

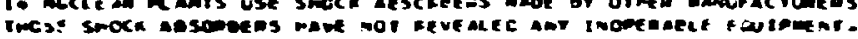

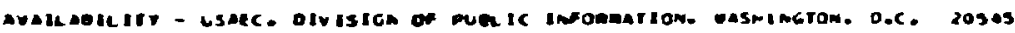

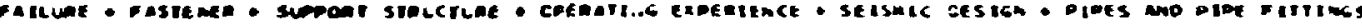

11,

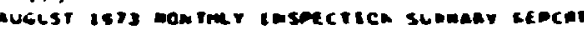

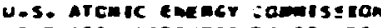

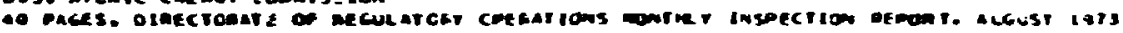

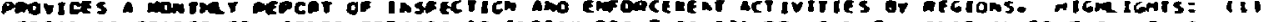

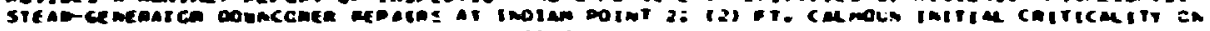

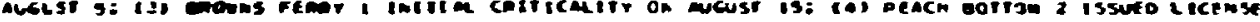

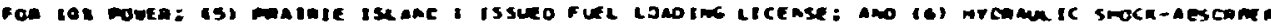
ancecte lochtifico.

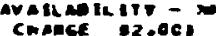

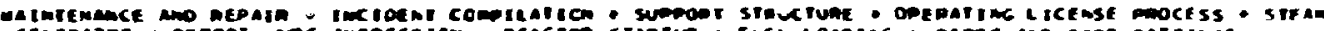

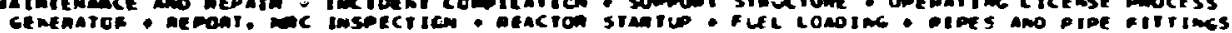

111

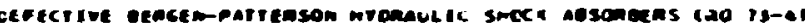

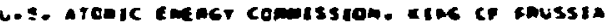

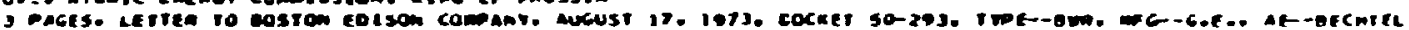

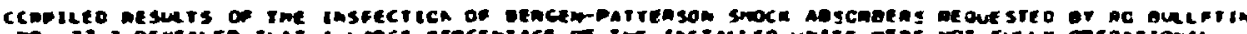

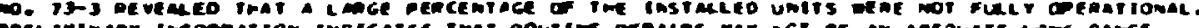

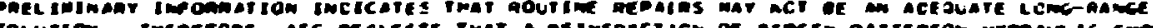

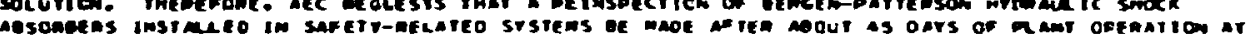

iesuegatine.

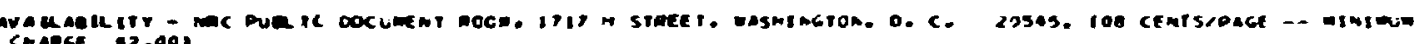

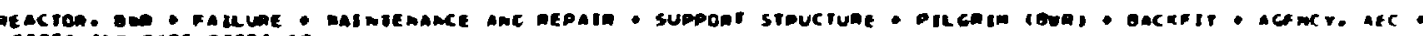
aiges anc cioe atirinss

112

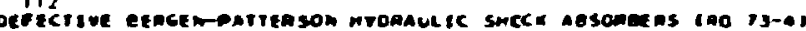

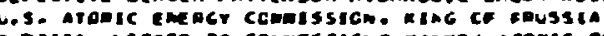

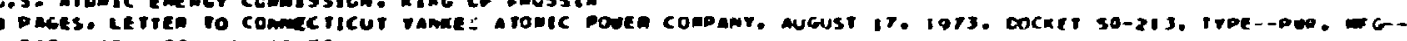
te si.. ace-siche atesien

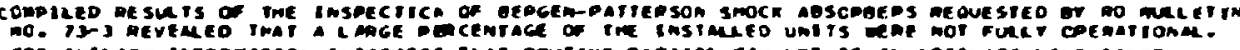

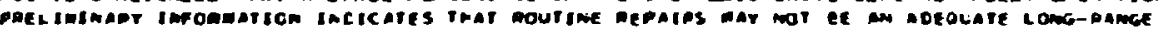

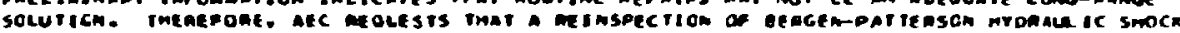

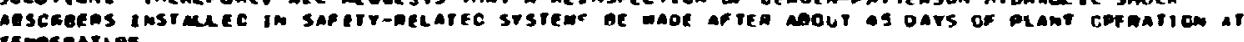
Temenaripe.

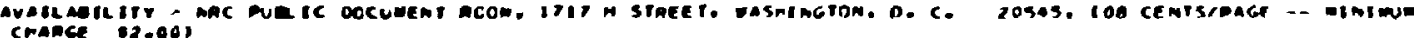

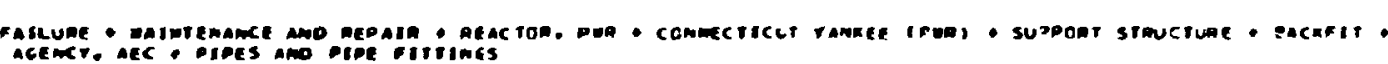

103 ."

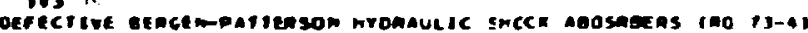

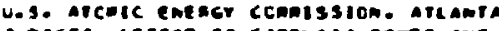

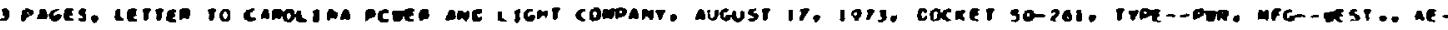
-eisce

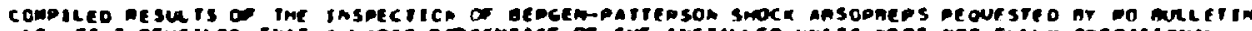

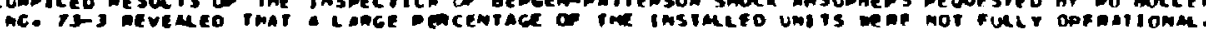

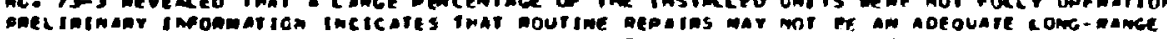

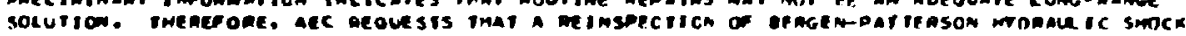

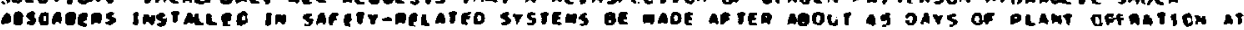
temberative.

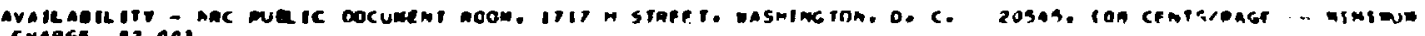
Crabe 12.001

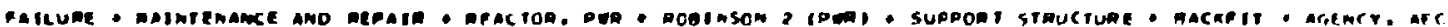


19

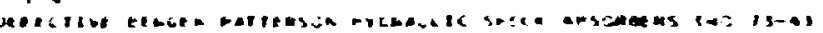

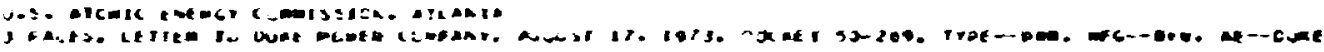

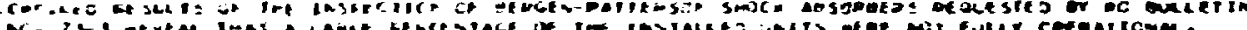

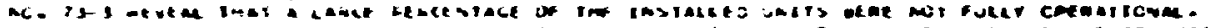

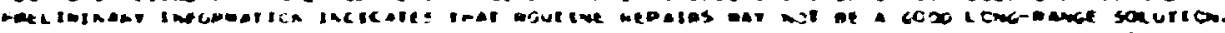

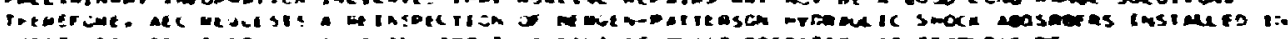

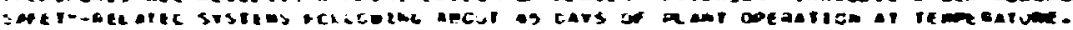

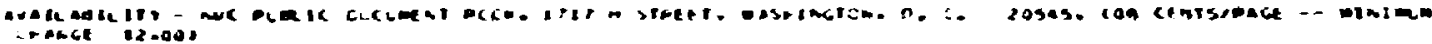

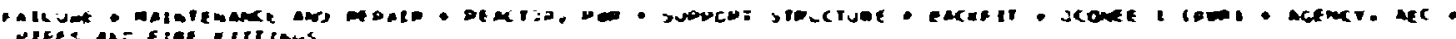

1.

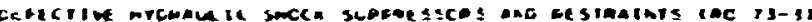

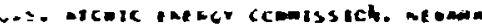

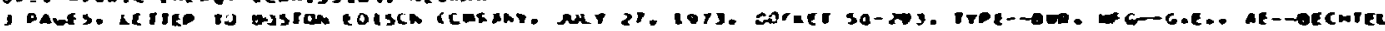

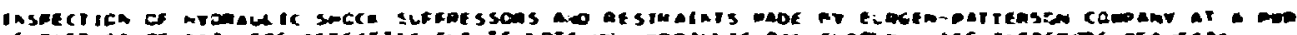

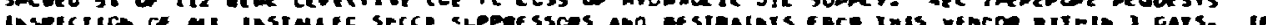

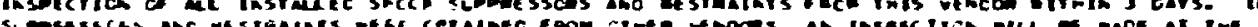
ne ir sturceien.

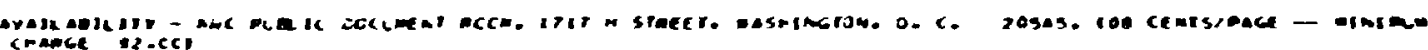

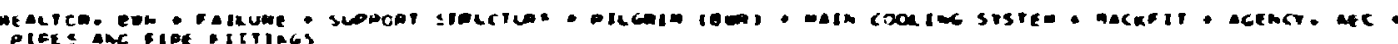

$\ln$

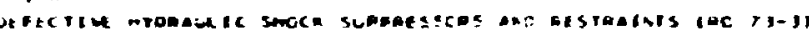

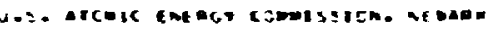

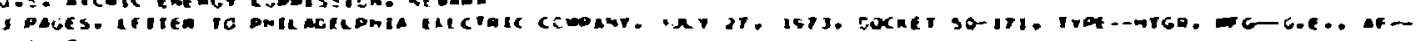
nicries

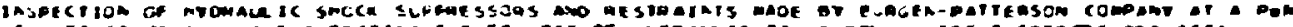

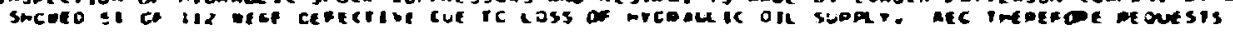

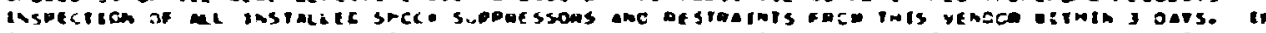

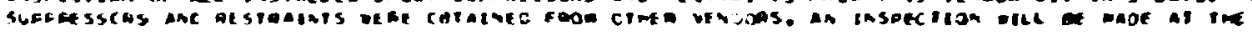
nets smuecen.

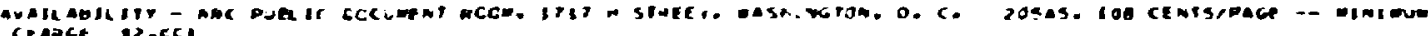

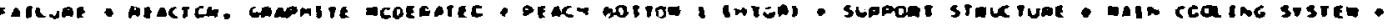

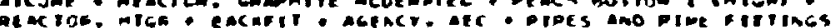

11 ,

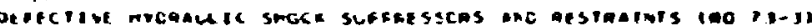

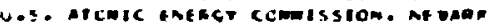

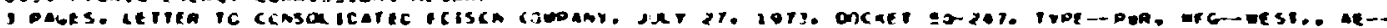

Nilic ence

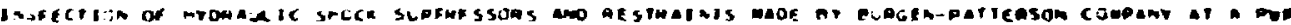

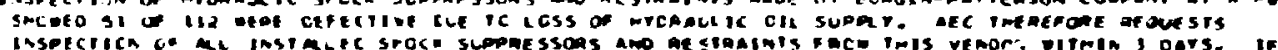

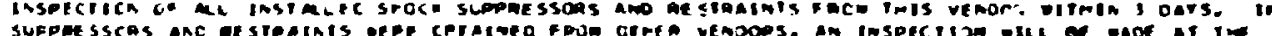
SWEDHE sscas anc

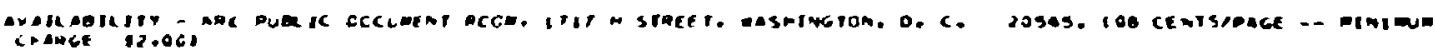

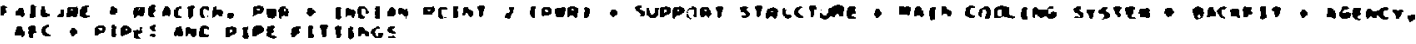

11

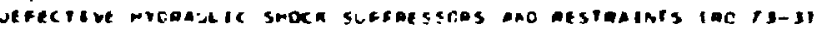

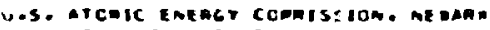

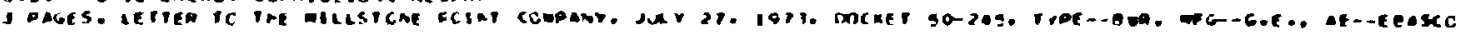

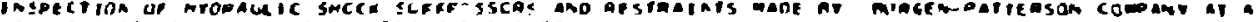

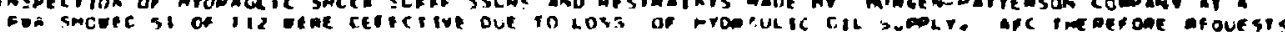

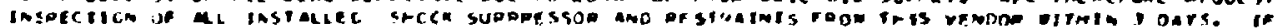

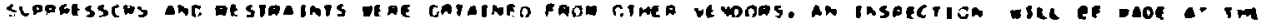
AF II Snciction.

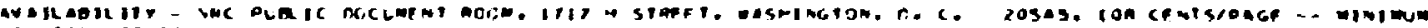
cratest 32001 


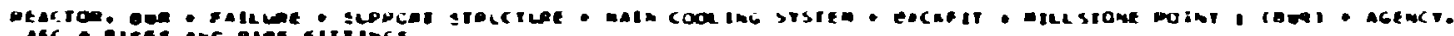
$12+$

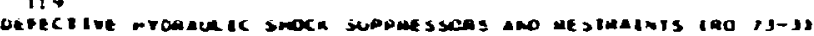

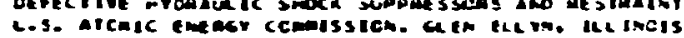

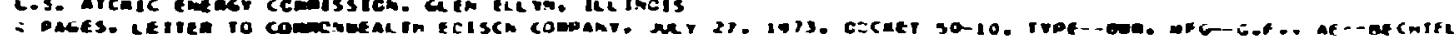

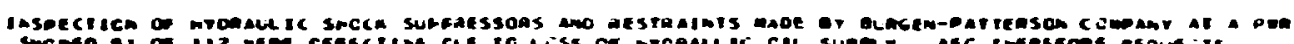

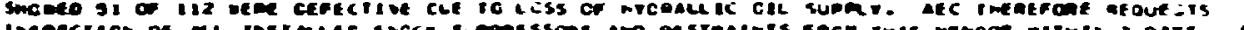

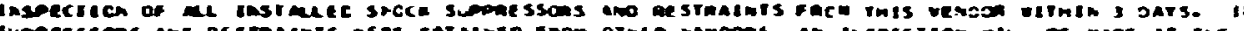
nexir spurcoun.

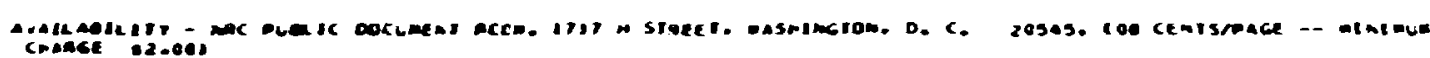

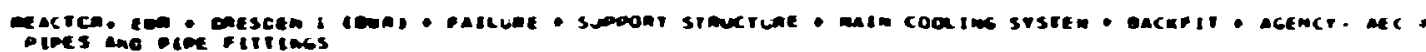

$1: 1$

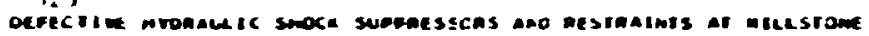

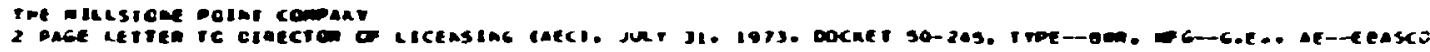

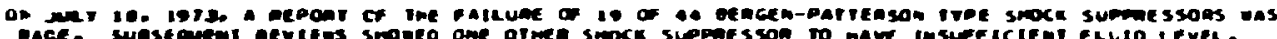

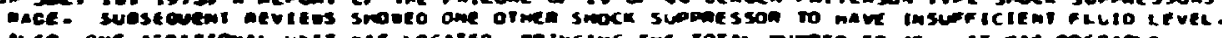

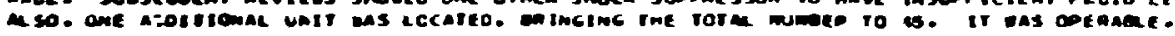

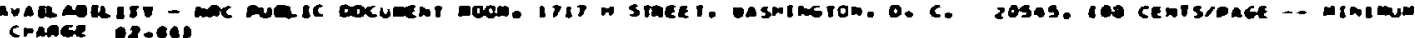

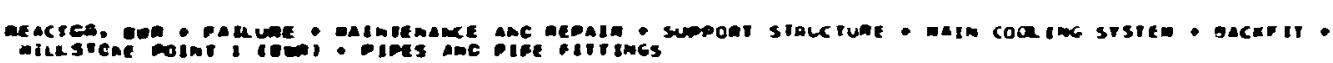

1,1

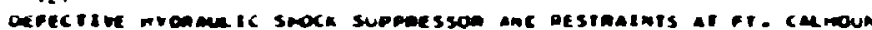

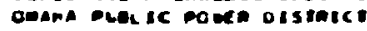

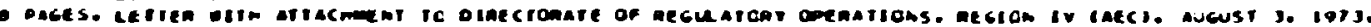

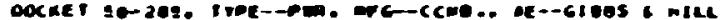

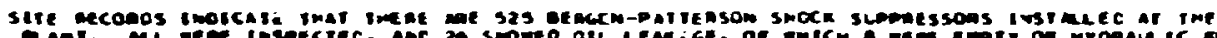

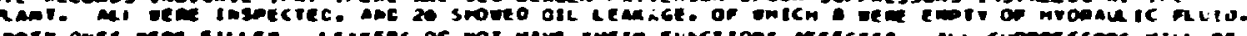

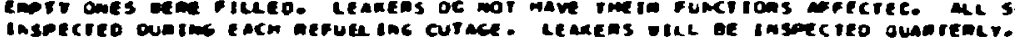

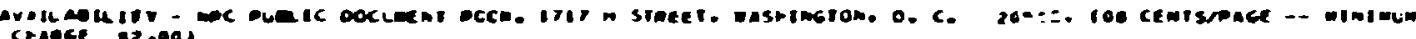

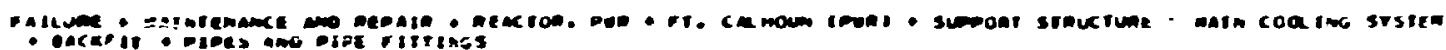

1.2

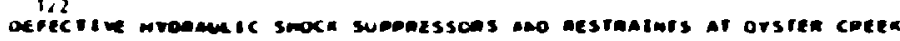

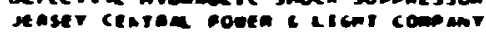

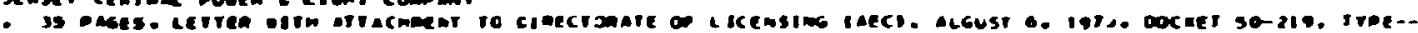

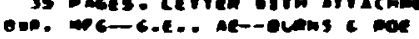

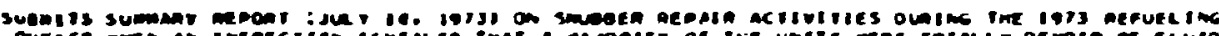

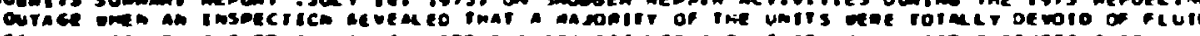

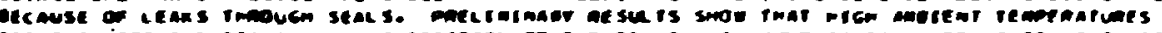

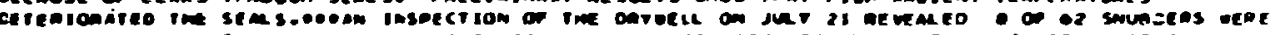

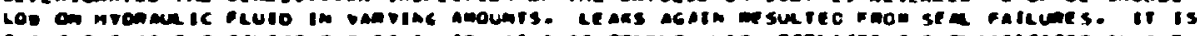

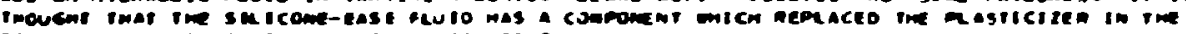

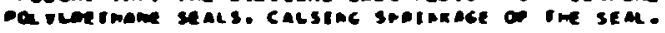

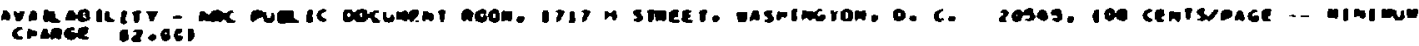

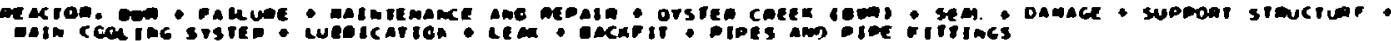

1,1

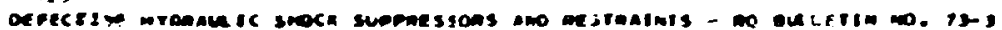

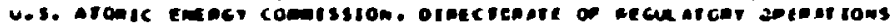

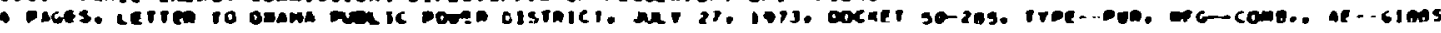
crice

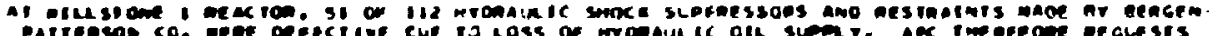

inescion co

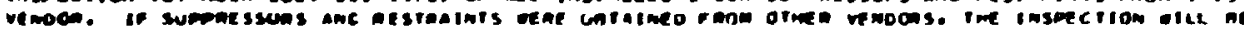

wade ai the mit smutocin.

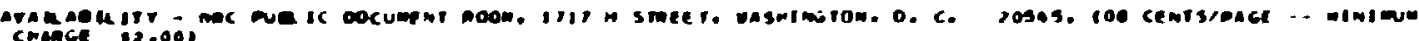




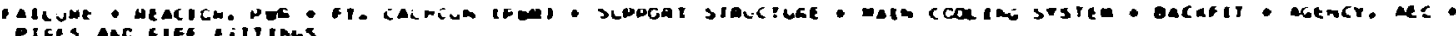

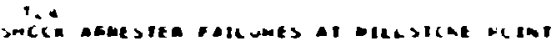

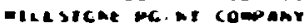

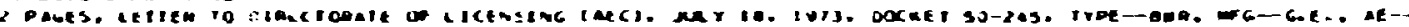
cousc

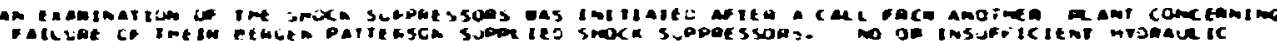

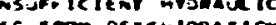

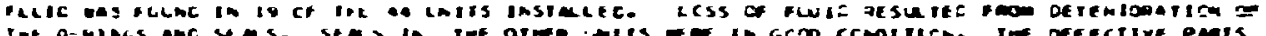

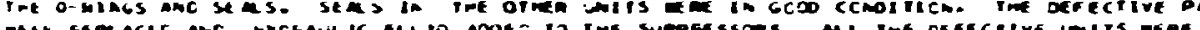

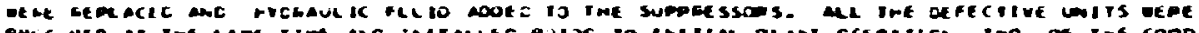

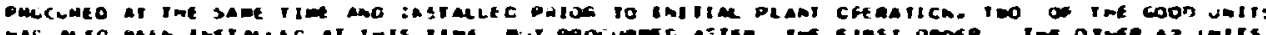
whe instince cared.

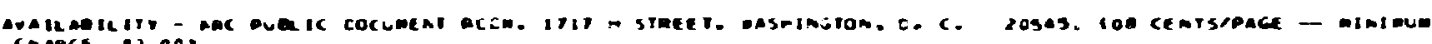

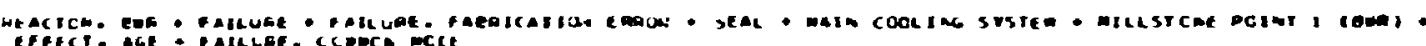

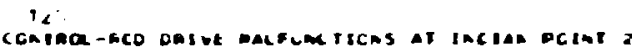

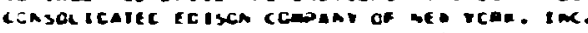

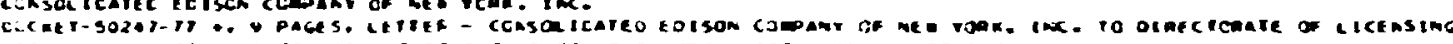

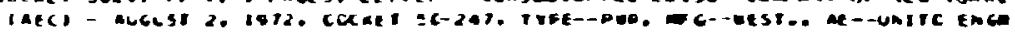

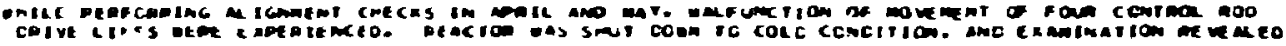

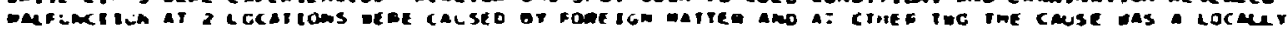

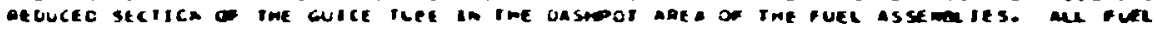

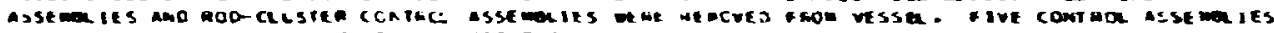

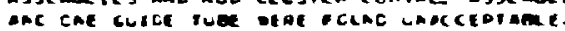

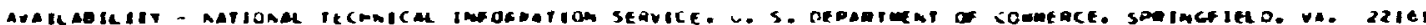

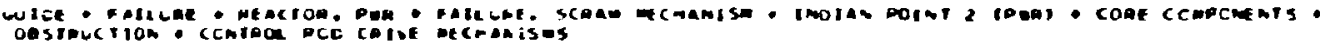

T.2

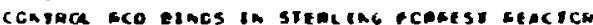

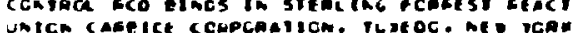

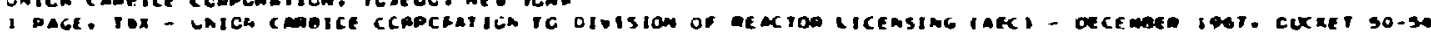

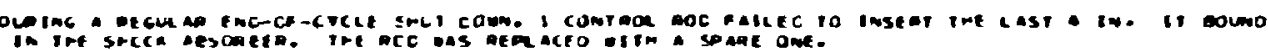

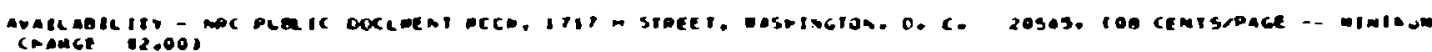

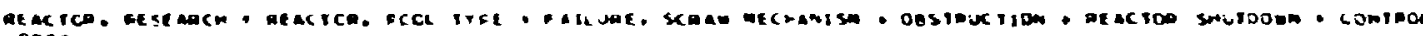
accs

$1: ?$

Secectec items ca inteats

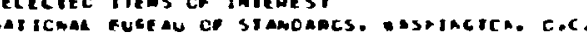

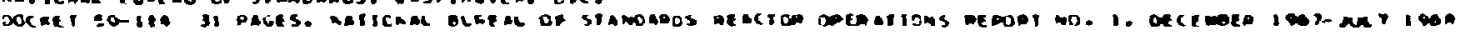

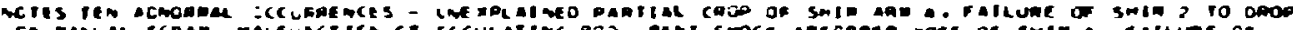

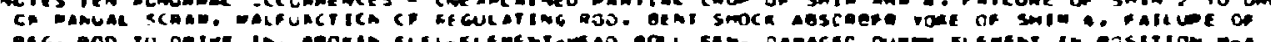

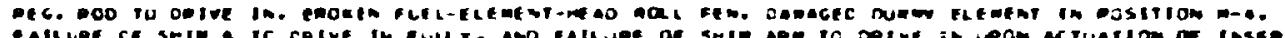

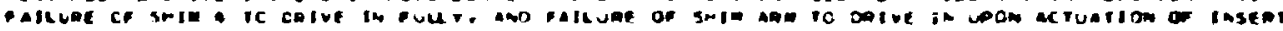
selere.

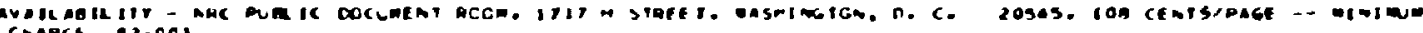
CrADGe 12.003

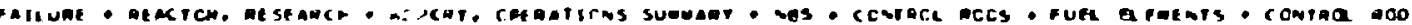
oe in eccontses

12

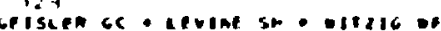

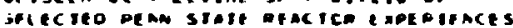

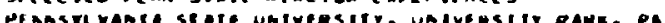

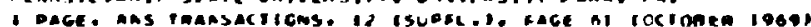

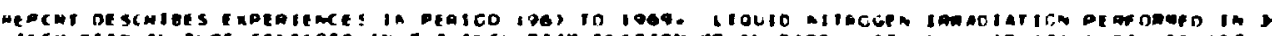

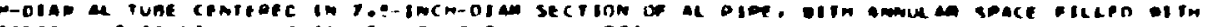

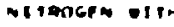

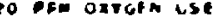

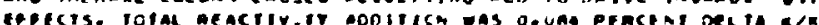




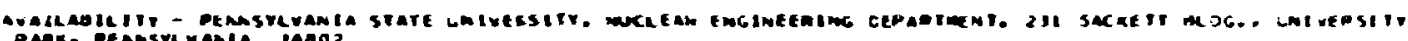

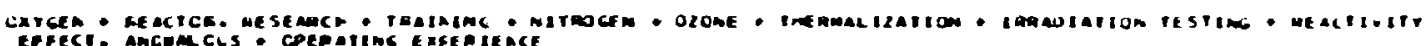

$1: 0$

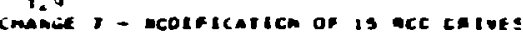

U.3. alcuic eneaci comissiom

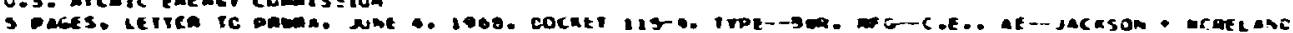

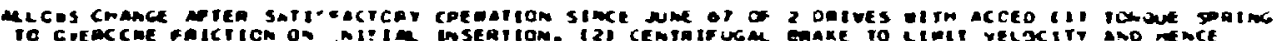

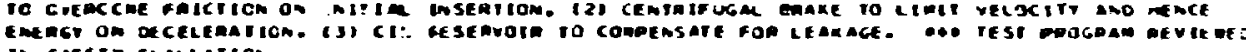

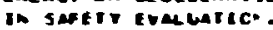

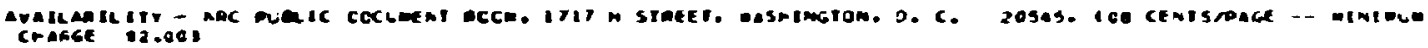

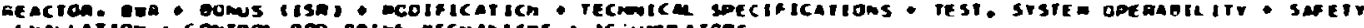

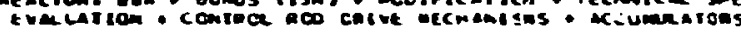

130

monset ca

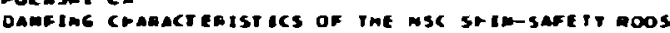

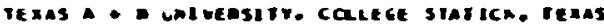

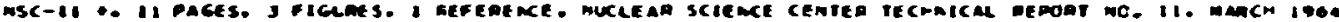

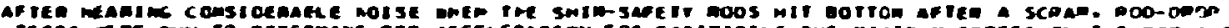

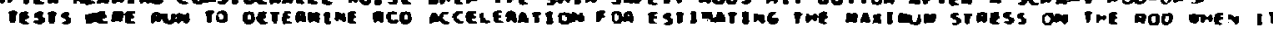

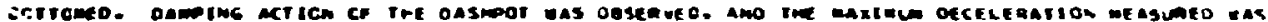

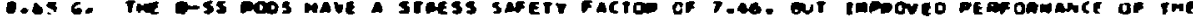
ousmaic mill ae invesilgatec.

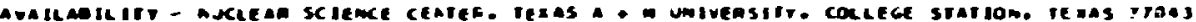

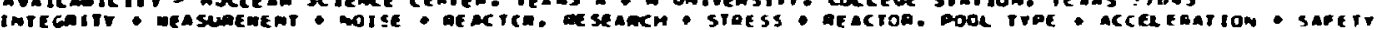

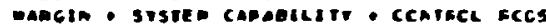

131

EEecreo ecals coearitinc enpepience

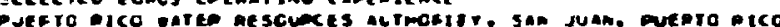

1.

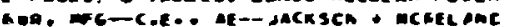

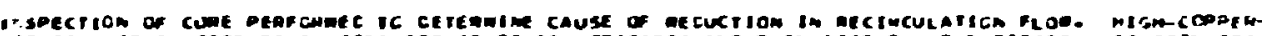

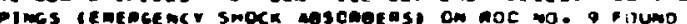

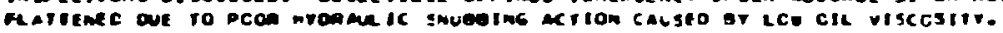

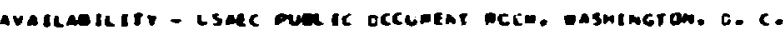

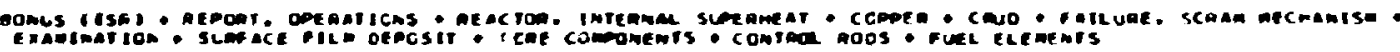


ince.

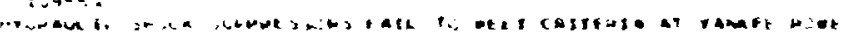

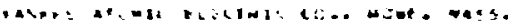

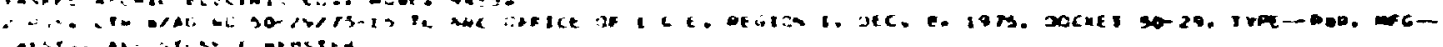

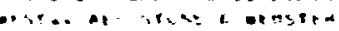

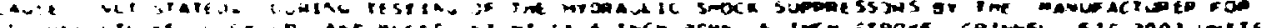

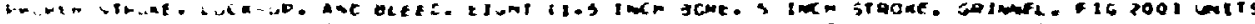

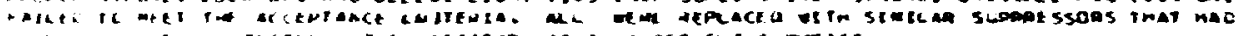

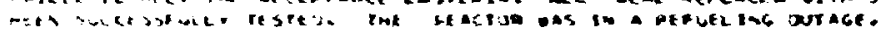

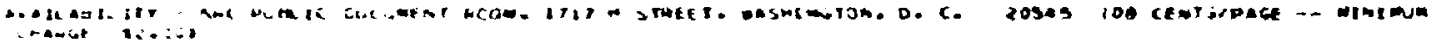

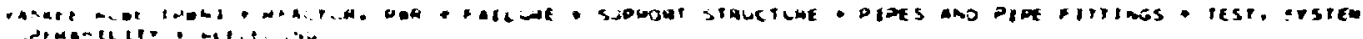

$1, \cdots, \cdots$

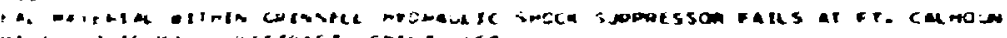

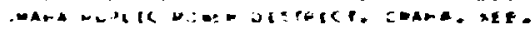

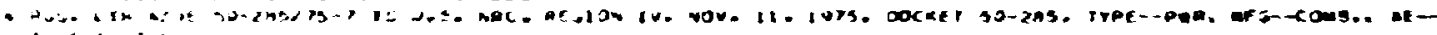

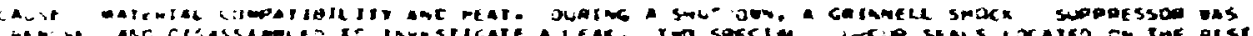

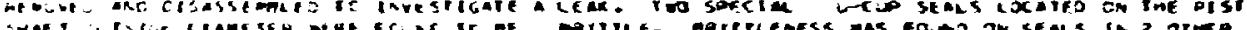
? Hat -Hi3. THe cut

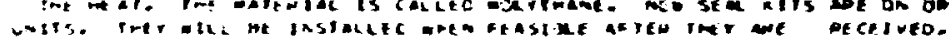

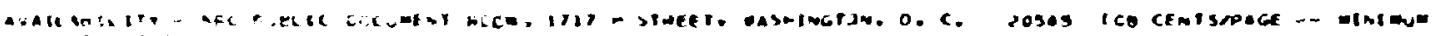

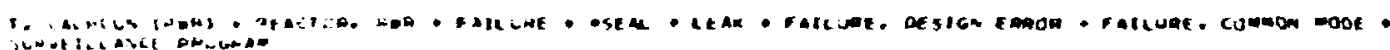

$1 . \div 203$

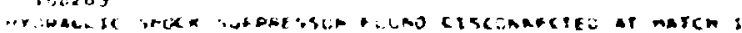

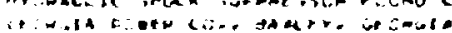

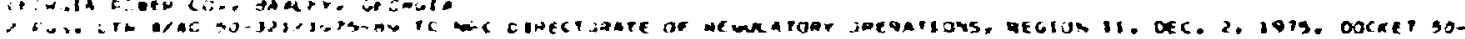

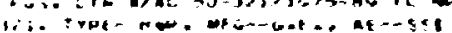

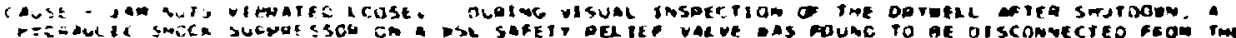

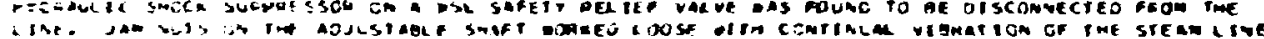

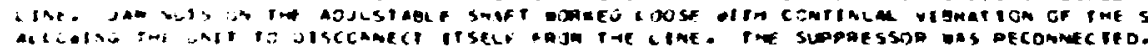

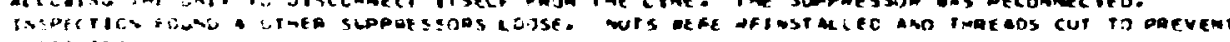
acista.

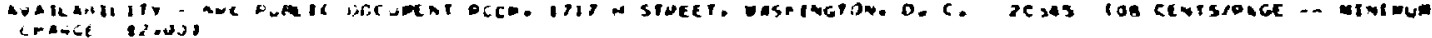

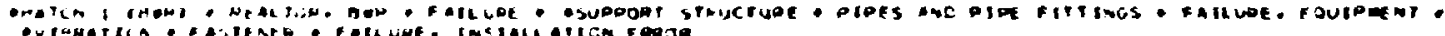

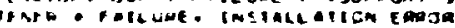

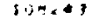

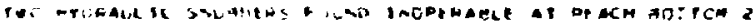

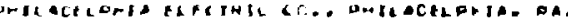

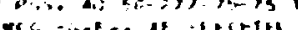

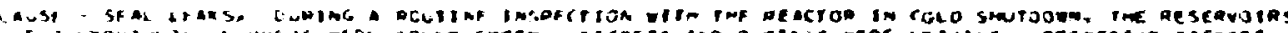

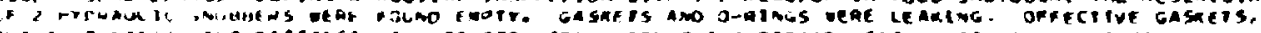

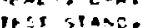

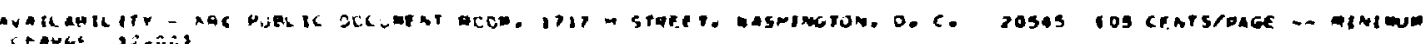

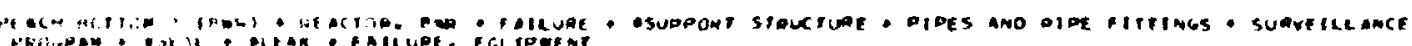

10400

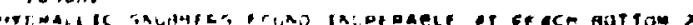

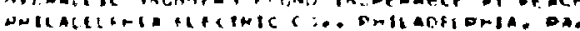

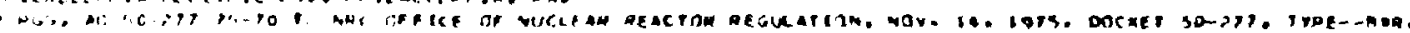

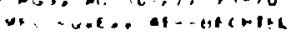

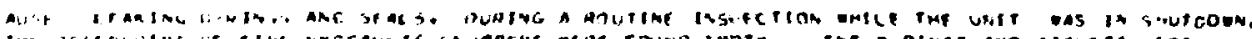

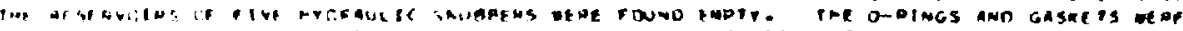

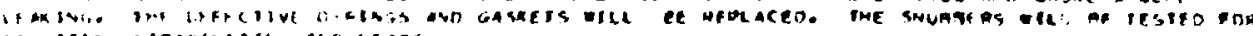

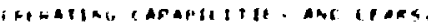




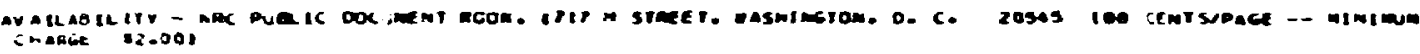

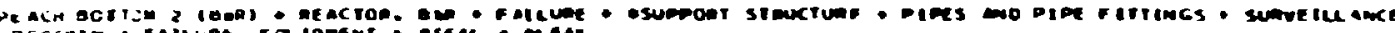

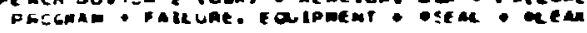

sionce

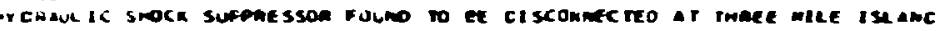

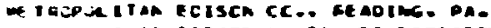

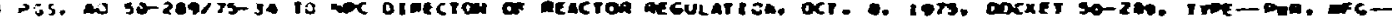
Hi.. AE--GILEETI ASSCC.

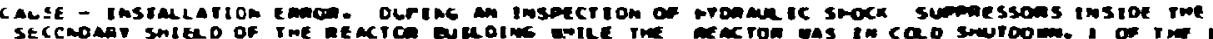

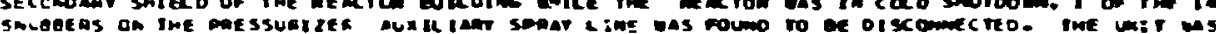

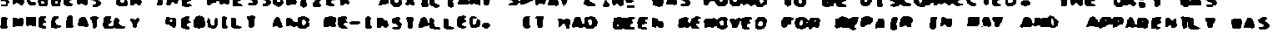

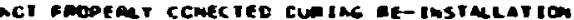

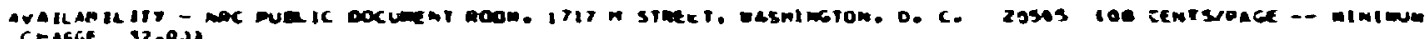

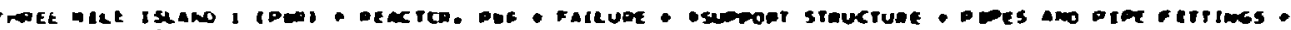

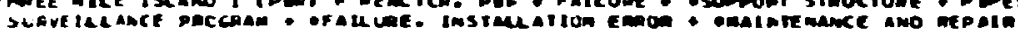


APPENDIX B

PRINTOLT OF RECON SEARCH OF NSA 


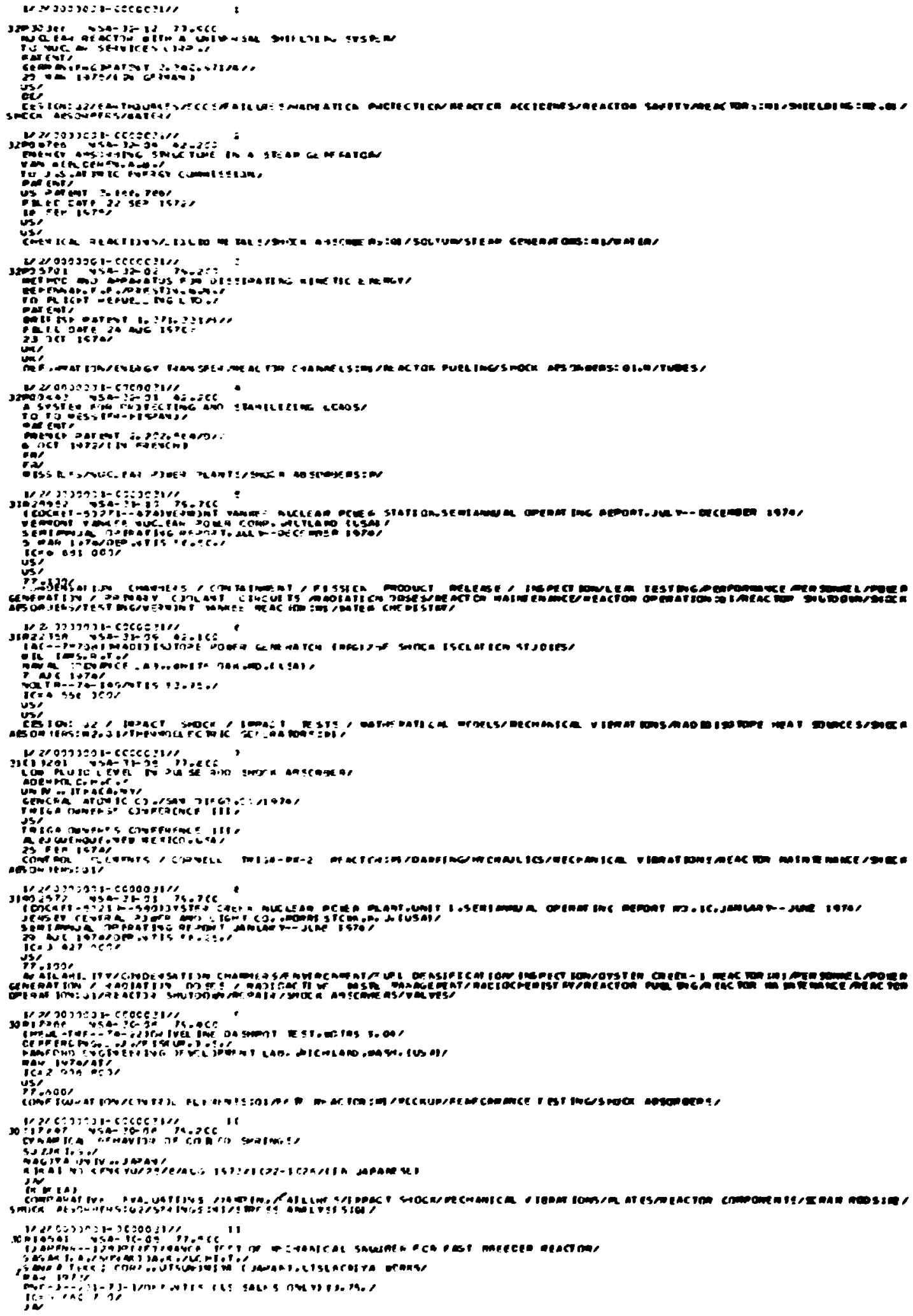




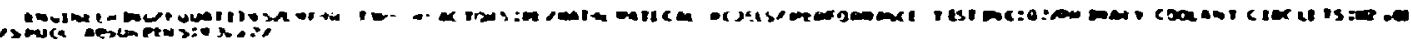
Dijo:

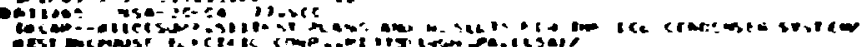

icion

Thas

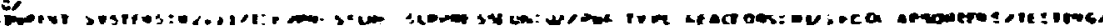

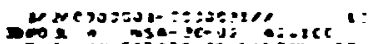

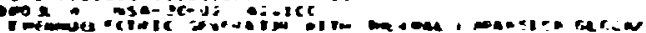

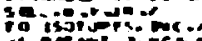

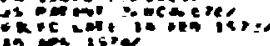

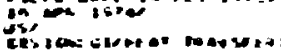

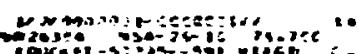

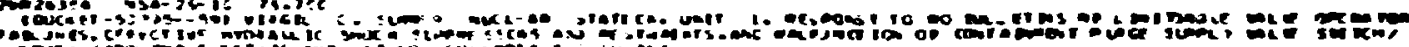

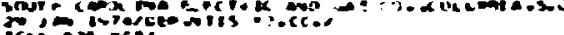

exis

Pove

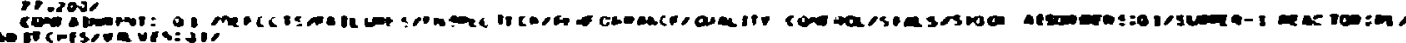

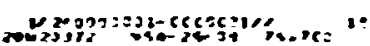

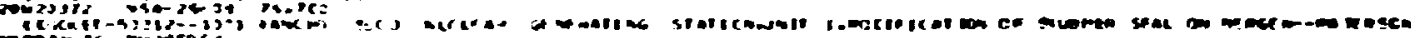

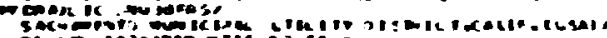

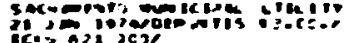

Ti:roos

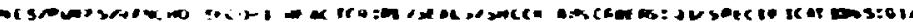

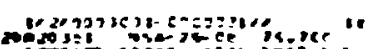

A wer

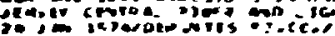

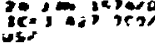

ister

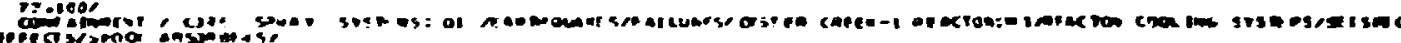

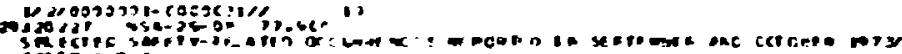

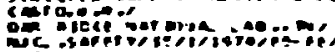

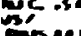

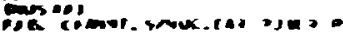

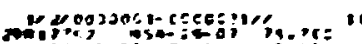

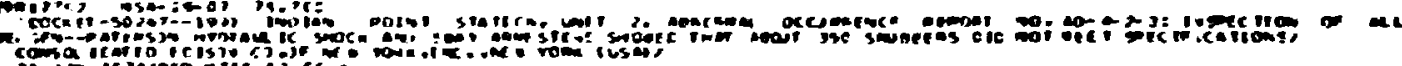

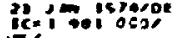

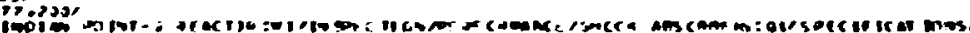

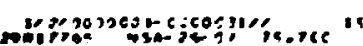

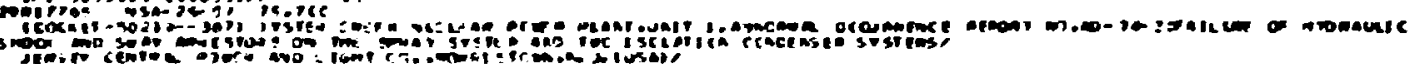

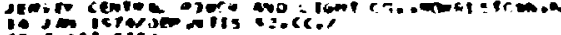

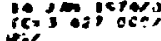

Yríl 30 ,

asoutinge

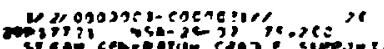

(1)

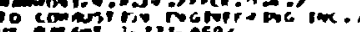

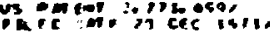

if ou iste

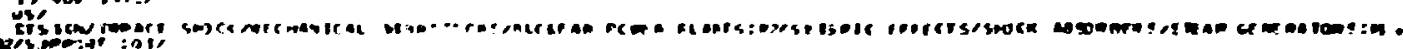

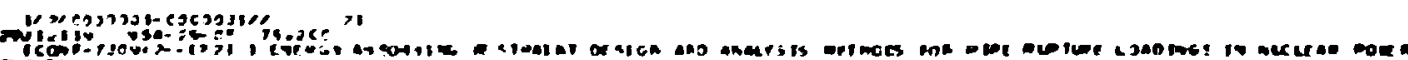

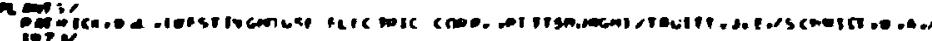

5i:

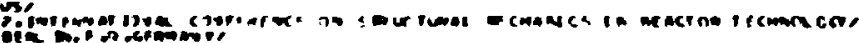

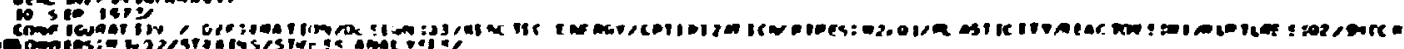

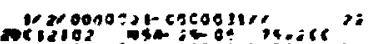

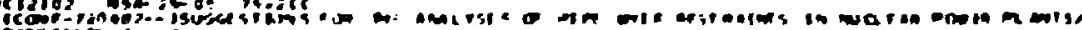

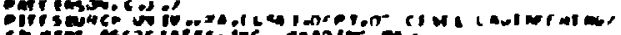

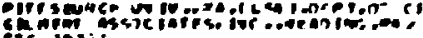

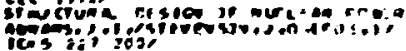

aches

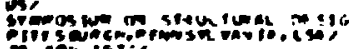

द्रि 100 : 


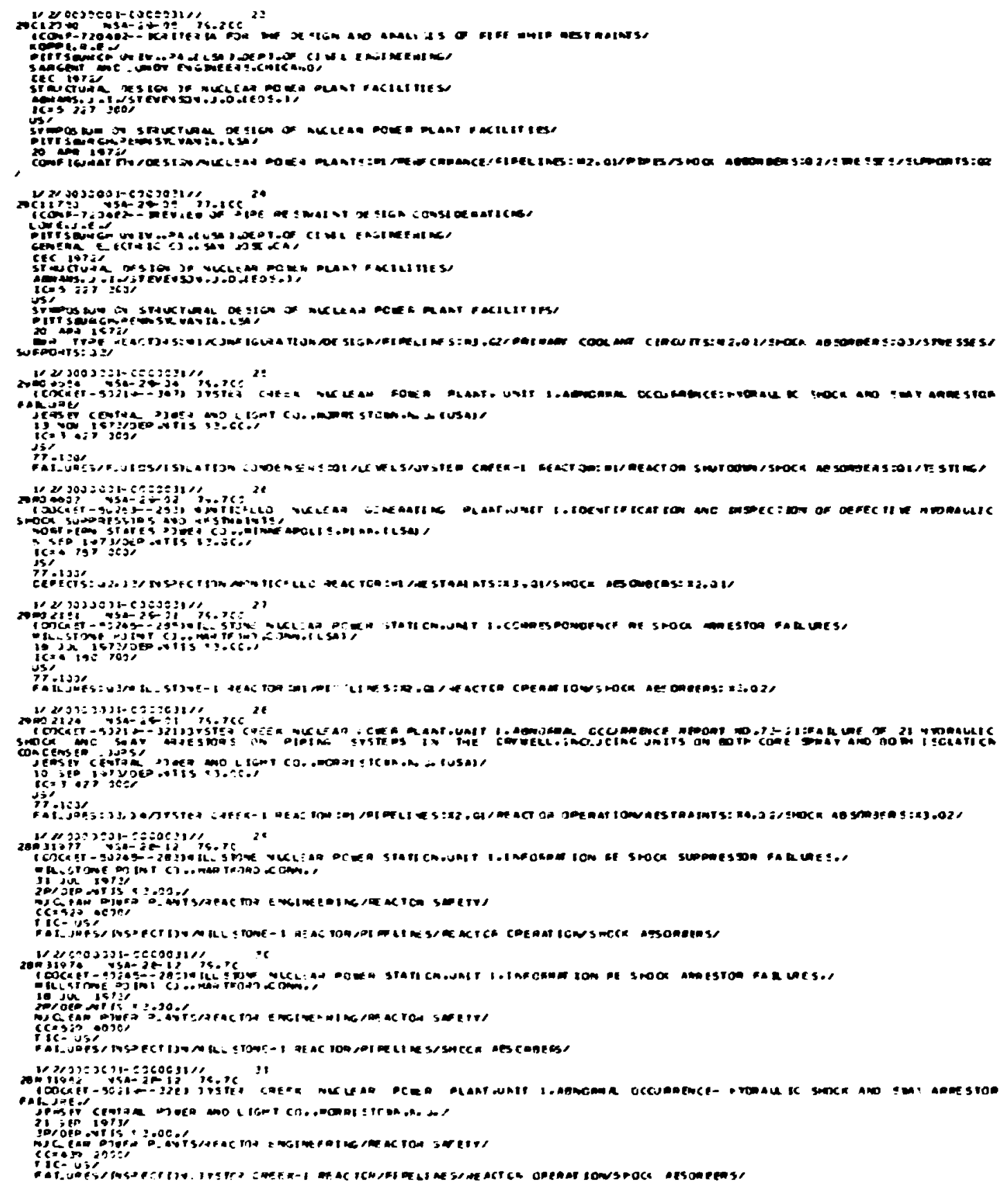


RO Bulletin No. 73-3

July 27, 1973

DEFECTIVE HYDRAULIC SHOCK SUPPRESSORS AND RESTRAITIS

Information contained in an abnormal occurrence report from the Millstone 1 reactor, supplemented by additional information obtained by Regulatory Operations from other licensees, revealed that 51 of 112 hydraulic shock suppressors and restraints inspected were defective due to loss of hydraulic oil sixply. These suppressors and restraints were manufactured by the Bergen-Patterson Company.

In the light of this information you are requested to take the followire specific actions:

A. Determine whether Bergen-Patterson hydraulic shock suppressors ard restraints are utilized in safety systems within your facility. Report the results of this determination to this office by noon, Monday, July 30, 1973.

B. If Bergen-Patterson stippressors and restraints are utilized in such systems:

1. Inspect prior to noon, July 30,1973 , ach suppressor and restraint for proper hydraulic fluid level.

2. Report by telephone to this office by noon, Monday, July 30, 1973, the results of your actions to show:

a. Number of suppressors and restraints installed in safetyrelated systems.

b. Number inspected.

c. Number defective by model number and location of installation and failure mode.

d. Confirmation of operability of defective suppressors and restraints. If repairs are not completed, your justification for continuing operation of your plant and your schedule for completion of repairs.

e. Existing or planned surveillance schedule for suppressors and restraints.

3. For planss with existing survelllance of suppressors and restraints, if your inspection has been performed within the last 30 days and there is no history of defective suppressors and 
restraints or if all were repaired, this request for inspection prior to noon July 30, 1973, does not apply.

C. Reports made in response to $A$ and $B$ above should be confirmed in writing within 10 days.

D. For facilities with no Bergen-Patterson suppressors and restraints or with existing surveillance inspections of suppressors and restraints:

1. At the next reactor shutdown, inspect all hydraulic suppressors and restraints in safety-related systems. Inform this office of your schedule for this inspection by noon, July 30, 1973.

2. When the inspection is performed, if defective suppressors and restraints are identified, the results of your inspection should be reported immediately by telephone to this office and confirmed in writing within 10 days. This report should include:

a. Number of suppressors and restraints inspected, identified by manufacturer.

b. Number of defective suppressors and restraints.

c. For each defectlve suppressor and restraint report:

(1) manufacturer

(3) location of installation

(2) model number

(4) failure mode

d. Confirmation of operability of defective suppressors and restraints. If repairs are not completed, your justification for operation of your plant and your schedule for completion of repairs.

e. Surveillance schedule.

3. If no defective suppressors and restraints are identified $q$ report should be submitted within 10 days of completion of the inspection to show the number of suppressors and restraints inspected identified by manufacturer and model number.

If you have any questions concerning this request, please contact this office. 


\section{APPENDIX I}

Regulatory Operations Bullet in No. 73-4

Date: August 17, 1973

DEFECTIVE BERGEN-PATTERSON HYDRAULIC SHOCK ABSORBERS

\section{INTORATITON}

Reference is made to Regulatory Operations Bulletin 73-3, dated July 27, 1973, entitled DEFECTIVE HYDRAULIC SHOCK ABSORBERS AND FESTRAINTS which provided information on known problems with hydraulic shock absorbers and which requested actions, including inspections of installed hydraulic shock absorbers, by certain utilities.

The compiled results of these inspecticns of Bergen-Patterson shock absorbers, based on telephone reports from affected licensees to the five Regional Offices of the Directorate of Regulatory Operations, reveal that a large percentage of installed Bergen-Patterson hydraulic shock absorbers were not fuily operational. In addition, preliminary information indicates that routine repairs, replacing defective seals, and filling hydraulic oil reservoirs may not be an adequate lorg-range solution to this problem. There is also some indication that deterioration of the affected hydraulic shock absorbers can occur over a relatively short period of time (less than 90 days).

ACTION INDICATED

1. Schedule and conduct a reinspection of Bergen-Patterson hydraulic shock absorbers installed in safety-related systems following approximately 45 days and no longer than 90 days after the plant has been at operating temperature subsequent to the inspection performed as requested in RO Bulletin 73-3.

2. The inspection should include:

a. Determination of condition of seals.

b. Verification of overall integrity of shock absorber assemblies.

c. Examination for loss of fluid, fluid leaks, and other signs of ilstrers.

d. Recording of piston rod extension and fluid level indicator positions.

3. Inform this office of the planned date for reinspeation of the BergenPatterson hydraulic shock absorbers. 
4. Provide this office with a prompt telephone report of the significant findings of this reinspection. If defective shocks are identified. a written report should be submitted to this office within 10 days following completion of the inspection; if no defective shock absorbers are identified, the written report st.suld be submitted within 30 days following the completion of the inspection.

5. The written report should include:

a. As appropriate, a description of the mode of failure, corrective action, and tests performed to determine the adequacy of the repair.

b. A description of the program for the development and subsequent installation of permanent sodification to the hydraulic shock absorber installation to assure proper operation. The description should include the technical basis for the conclusion that deficiencies in the Bergen-Patterson hyaraulic shock absorbers have been resclved. 
APFENDIX E

IE Bulletin No. 75-05

April 14, 1975

OPERABILTIY OF CATEGJRY I HYDRAULIC SHOCK ANE STAY SUPPRESSORS

DESCRTPION OF CIRCUMTANCES:

The Metropolitar Edison Company notified the Region I Office of Inspection and Enforcement by telephore on March 21, 24, and 25, 1975, of the finding of eight inoperable hydraulic shock suppressors on the wain steam lines inside containment at the Three Mile Island 1 facility.

The licensee reported that these suppressors were removed for overhaul 'seal replacement) and testing during a planned maintenance program. The suppressors were determined to be inoperable (suppressor lockup could not be achieved during piston displacement tests perforred by the licensee. The tests were performed on a test rig which actuates the pistor. with air pressure. These suppressors, representing equipment originally installed at the facility, were specified to achieve lockup at a velocity not exceeding $10 \mathrm{in}$. per minute. The test rig at the facility was reported to have a capability of testirg at velocities up to 50 in. per minute. The specific cause of the reported failures has not been determined. Spare suppressors were installed as replacements after their acceptability was determined by the licensee by test.

FCr your information, Ro Bulletins $73-3$ and $73-4$, dated July 27, 1973, ard August 17, 1973, respectively (copies enclosed), discuss some prior experience concerning the operability of hydraulic suppressors.

In gexeral, the installation of hydraulic suppressors provides a system for the restraint of category I systems and components against excessive movement during seismic and fluid system transient conditions. Although in such a restraint system the fallure or inoperability of a single suppressor would not, normally dereat the design function of the rests aint system, it is di sirable to provide for the periodic testing of a representative sarp] : from the tctial population of suppressors to assess the operational ca. ability of the restraint system on a continuing basis.

\section{ACTION TO BE TAKENN BY LICENSEES:}

1. For all power reactor facilities with an operating license:

Review the design and installation of your hydrailic restraint systems, and provide the following information to this office in writing within 30 days of the date of this Bulletin: 
a. The design requirements which the various suppressors are intended to meet, such as velccity, acceleration, load, etc. Also, indicate the margin available between the design requirements ant the requirements spectfied for purchase of these components.

b. Describe the testing of the hydreulic suppressors conducted by you or your supplier(s) prior to installation to essure their operation in accordance with design requirenents.

c. Describe the surveillance (including testing) programs now underway or planned by you to assure contimued operability of the hydraulic suppressors under the design conditions throughout the Iffe of the facility.

2. For all power reactor facilities with a construction permit:

Report to this office in writing within 60 days of the date of this Bulletin or prior to completion of preoperational testing, whichever is earlier, the information requested in Iters 1.a. through 1.c. abore.

A copy of your response to Itens 1 and 2 above should also be sent to: Assistant Director for Construction and Operations, Office of Inspection and Enforcement, USIRC, Washington, D.C. 20555. 


\author{
APFDIDDX $F$ \\ Questionnnaire Sent to Reector Operators \\ OAK RIDGE NATIONAL LABORATORY \\ openateo or \\ union cancioe conponatiom \\ nuclear division \\ OAK RIDCE. TEMnESSEE 3700
}

December 3, 1975

Mr. I. R. Finfrock, Jr.

Vice President

Jersey Central Power and

Light Co.

Madison Avemue at Punch Soull Rd.

Morristown, NJ 07960

Dear Sir:

I am presently investigating, under the auspices of ARC, the fallure criteria of hydraulic sbock absorbers (smibbers) as used in reactor facillties across the country. It is my understanding that you reported a sumber fallure in the letter('s) listed below as indicated by the dates they were received by ERDA along with the associated Docket mmber. It would be a great aid to mo Investigation if you could supply we with aw test data or evaluations you, the smubber mampacturer and or an independent laboratory, performed regarding performance or mode of raslure related to falled smubber(s) as indicated in the below A.0. report(s). Your cooperation will be greatly appreciated.

Sincerely yours,

J. H. Butler

Reactor Division

(615) $483-8611$, Ext. 3-7185 or 3-5509

$\mathrm{JHB} / \mathrm{lad}$

Reactor Docket

Oyster Creek Unit $1 \quad 50-219$
50-219 Aug. 6, 1973; Sept. 10, 1973;

Sept. 21, 1974; Nov. 13, 1973;

Jan. 234 (sic), 197t; Feb. 19, 1974;

Mar. 18, 1974; Apr. 26, 1974; May 29,

1974; July 16, 1974; Sept. 23, 1974 